$x: 6$.

Cable Sarrey.

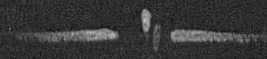

$2236-\operatorname{Tor}: 18=18$

$1286-\operatorname{dec}{ }^{r} 2: 188 t$ (2) 


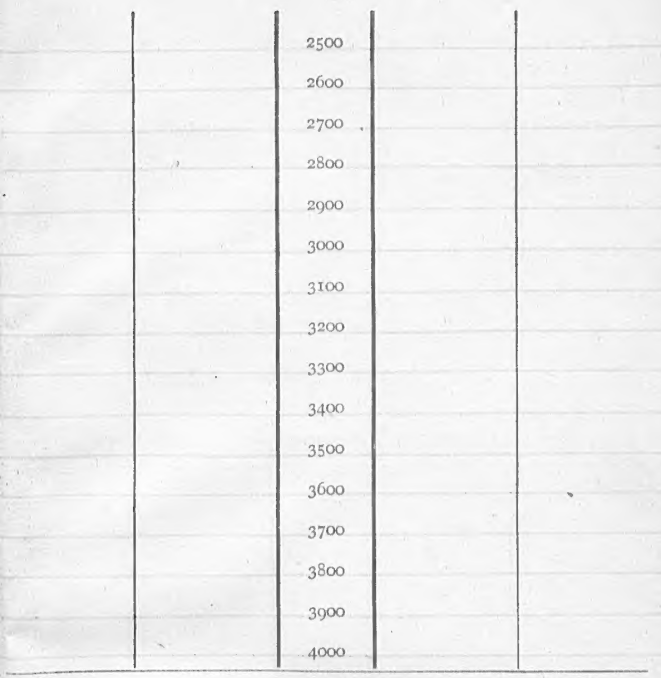

SERIAL TEMPERATURES.

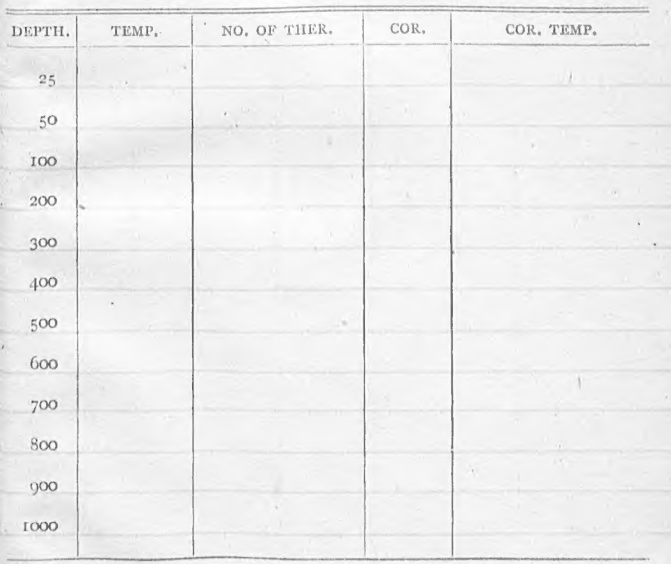

REMARKS : 
Rn

9.

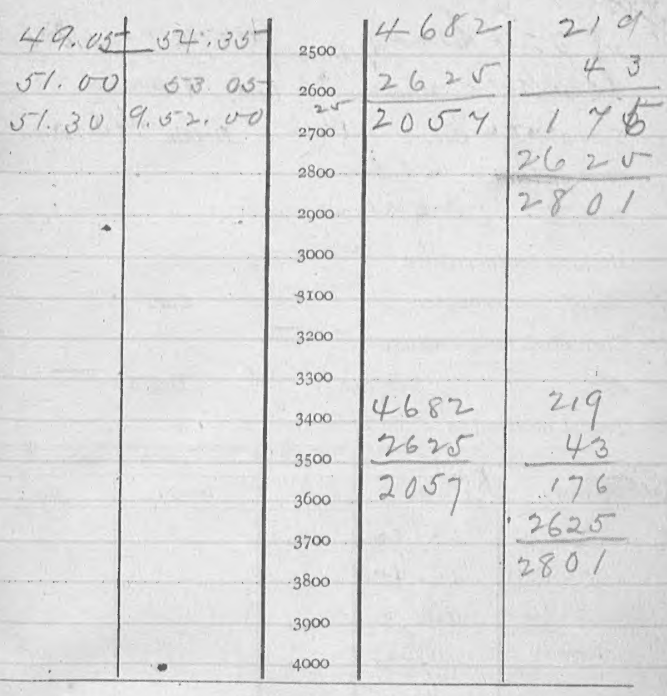

SERIAL TEMPERATURES.

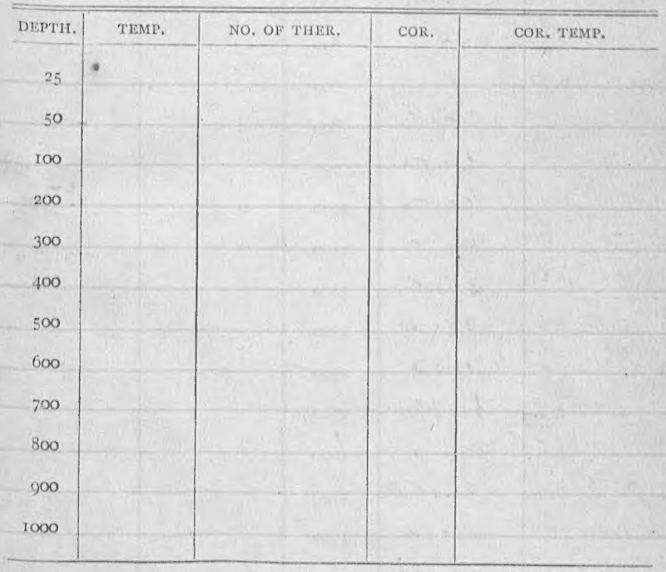

REMARKS :

Nothing but clear water mi cup. 
$23043^{\prime} \mathrm{H} .16^{\circ} 3^{\circ} 31.30^{\prime \prime} \mathrm{rr}$.

ini. $=2893$

so. 237 eluncy Date Grom $/ 518-154$

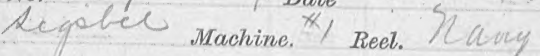

Turns 2574 Cor. +174 Depth 2948 fuw.

Shot-ortewd 60 elo.

Bottom

Bottom temperature

No. of thermometer

Correated temperature

Air $\quad 75$

Surface $)^{4}$

Drift

\section{Trawt or dredge}

SOUNDING WIRE. QN DOWN. I UP.

$1 / 142 \cdot 20$

$43.4 v \quad 42-45$

$44.30 \quad 42-00$

$40.35 t \quad 41.20$

$4650 \quad 40-40$

$4800] 40-00$

$49.05^{-} \quad 39-05$

so. $05-38-10$

$51.15-37-15$

$52 \cdot 30$

\begin{tabular}{l|l}
53.45 & $35-00$ \\
55.10 & $34-00$
\end{tabular}

$36-00$

$3-6.30 \quad 33-00$

3800

- $32-05$

the 59.25

$12-01-00$

$02-15 \quad 29-15$

$03-3 \otimes 28-15$

$05=00$

$06-15$

$07-35$

$09-00$

$10-30$

$12-00$

$13-30 \mid 12-21-00$

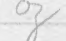

8
Cor.

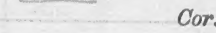


tim, tovil9

\begin{tabular}{|c|c|c|c|c|}
\hline $15-00$ & $1.19-45$ & 2500 & 4682 & $=19$ \\
\hline \multirow[t]{15}{*}{$16-15$} & $12-18-45$ & $\begin{array}{l}20574 \\
2600\end{array}$ & 2574 & 45 \\
\hline & & 2700 & 210.8 & 174 \\
\hline & & 2800 & & 2574 \\
\hline & & 2900 & & 2748 \\
\hline & & 3000 & & \\
\hline & & 3100 & & \\
\hline & & 3200 & & \\
\hline & & 3300 & & \\
\hline & & 3400 & $10^{\circ}$ & \\
\hline & & 3500 & 4682 & 219 \\
\hline & & 3600 & 2574 & 45 \\
\hline & & 3700 & 2108 & 174 \\
\hline & & 3800 & & 2574 \\
\hline & & 3900 & & 2748 \\
\hline & & 4000 & & \\
\hline
\end{tabular}

SERIAL TEMPERATURES.

\begin{tabular}{r|r|r|r|r}
\hline DEPTH. & TEMP. & NO. OF THER. & COR. & COR. TEMP. \\
\hline 35 & & & & \\
50 & & & & \\
100 & & & & \\
200 & & & \\
300 & & & \\
400 & & & \\
500 & & & \\
600 & & & \\
700 & & & \\
800 & & & \\
\hline 000 & & & \\
\hline
\end{tabular}

REMARKS : 


\section{4}

No. 238 Sherey Date how? 19-1891. digebe Machine." Reel. Mary.

Turns 2458 Cor. +169 Depth 2627 fuse Shot orederd 60 thes.

Bottom no sfoccimen

Bottom temperature 35,6

No. of thermometer $54 \quad 8 \quad 15$ Cor. -0.3

Corrected temperature 35.3

Air 75 Surface 75 Drift -

Trowl or dredge -

\begin{tabular}{|c|c|c|c|c|}
\hline Xnd SOUND & ING WIRE. & & DREDGE & OPE. \\
\hline Down. & UP. & TURNS. & Down. & UP. \\
\hline$-02-15$ & $01-00$ & o & & \\
\hline $03-30$ & $3-00-10$ & 100 & & \\
\hline $04-30$ & $59-20$ & 200 & & \\
\hline $05-30$ & $58-30$ & 300 & & \\
\hline $06-30$ & $57-30$ & 400 & & \\
\hline $07-30$ & $56-45$ & 500 & & \\
\hline $08-35$ & $56-00$ & 600 & & \\
\hline $09-40$ & $55-00$ & 700 & & \\
\hline $10-45$ & $54-00$ & 800 & & \\
\hline 12.00 & $52-45$ & 900 & 3 & \\
\hline $13-15$ & $51-30$ & 1000 & 5 & $c^{2}$ \\
\hline $14-30$ & $50 \cdot 15$ & rroo & 5 & \\
\hline $15-45$ & $49-15$ & 1200 & S & \\
\hline $17-00$ & $48-15$ & 1300 & $q^{6}$ & \\
\hline $18-30$ & $47-15$ & 1400 & \} & \\
\hline $20-00$ & $46-15$ & 1500 & & \\
\hline $21-20$ & $45-15$ & 1600 & $\beta$ & \\
\hline $2 z-40$ & $44-15$ & 1700 & $\neq \quad 3$ & \\
\hline $24-50$ & $43-15$ & $x 800$ & $\therefore \quad 2$ & 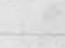 \\
\hline $25-30$ & $42-15$ & 1900 & & \\
\hline $27-00$ & $41-15$ & 2000 & 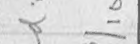 & \\
\hline $28-30$ & $40-15$ & 2100 & & \\
\hline $30-00$ & $3 q-15$ & 2200 & & \\
\hline 31.30 & $38-15$ & 2300 & 53 & . \\
\hline $33-00$ & $2-37-15$ & 2400 & & \\
\hline
\end{tabular}


tim.

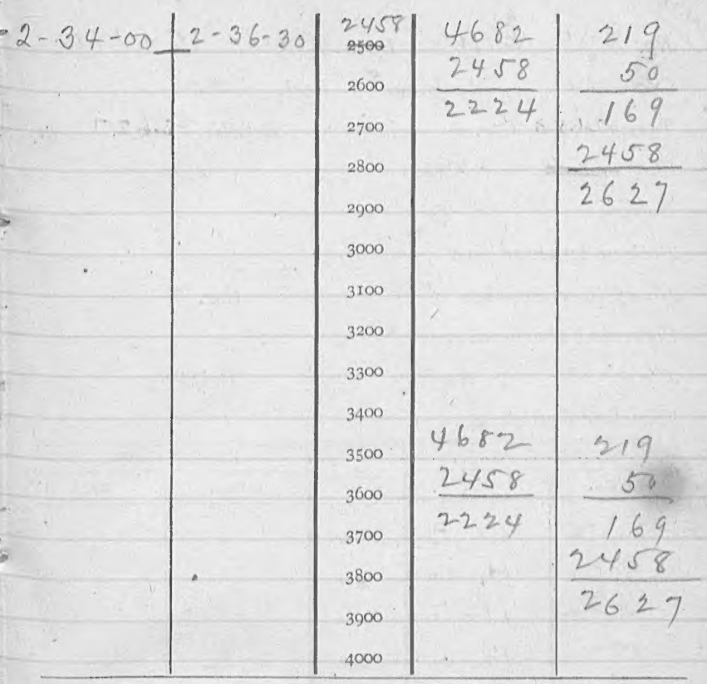

SERIAL TEMPERATURES.

\begin{tabular}{r|r|r|r|r}
\hline \hline DEPTH. & TEMP. & NO. OF THE, & COR. & COR. TEMP. \\
\hline 25 & & & & \\
50 & & & & \\
100 & & & & \\
200 & & & \\
300 & & & \\
400 & & & \\
500 & & & \\
600 & & & \\
700 & & & \\
800 & & & \\
900 & & & \\
1000 & & & \\
\hline
\end{tabular}

REMARKS :

Nothing but clear water mi cap. 
2895

No.

instr

Machine. Reel.

Turns Ny 4 Cor. +

168

shot or 60 els

Depth $26 / 10$

Bottom

bi 03

Bottom temperature -

Sro. of thermometer

Cor. -

Corrected temperature

Air

Surface

76

Drift

Trawl or dredge

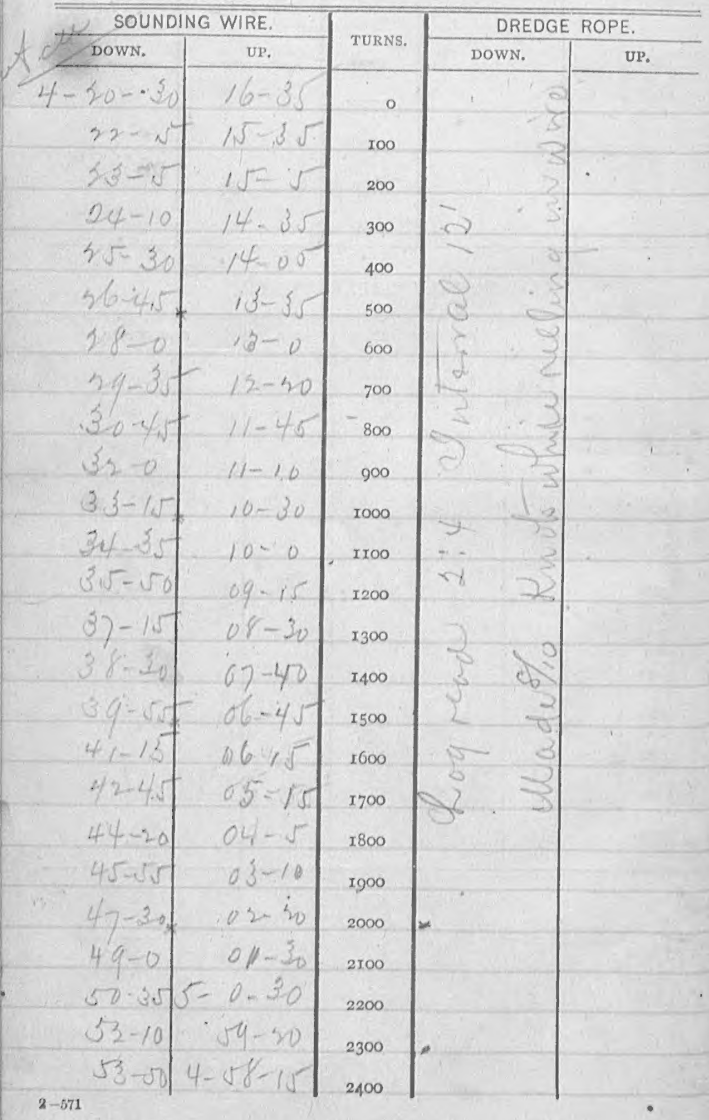




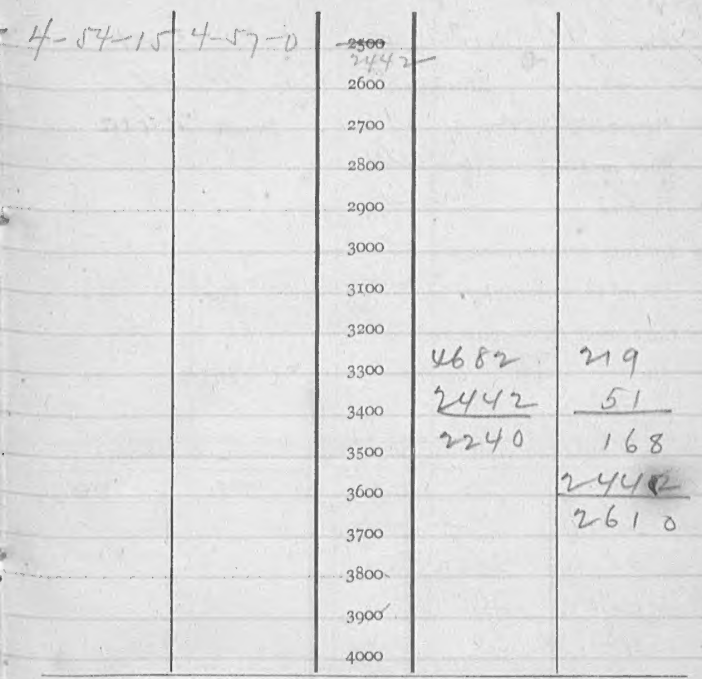

SERIAL TEMPERATURES.

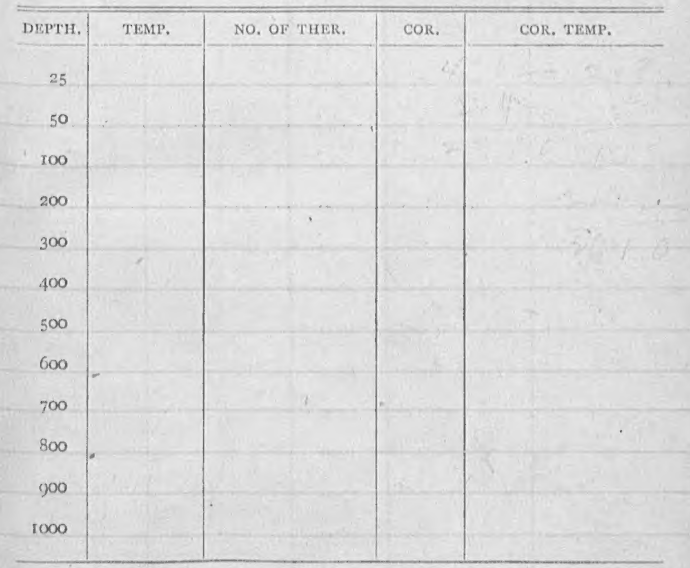

REMARKS: 
No.

\section{Date}

Machine. , Recl.

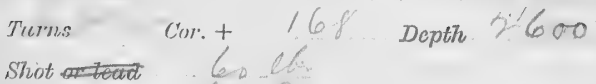

\section{Bottom}

Bottom temperature

No. of thermometer. 54

Cor.

Correctod temperature 35 .

Air........ $]^{6}$ Sterface . Jirift

Trawl, or diectso

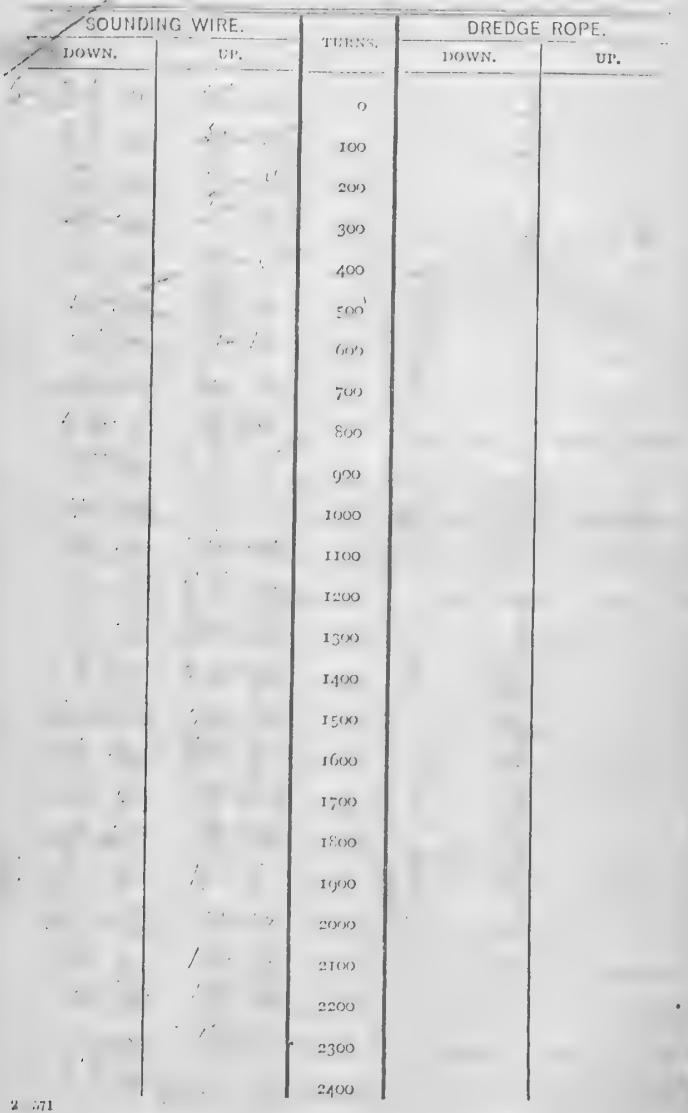




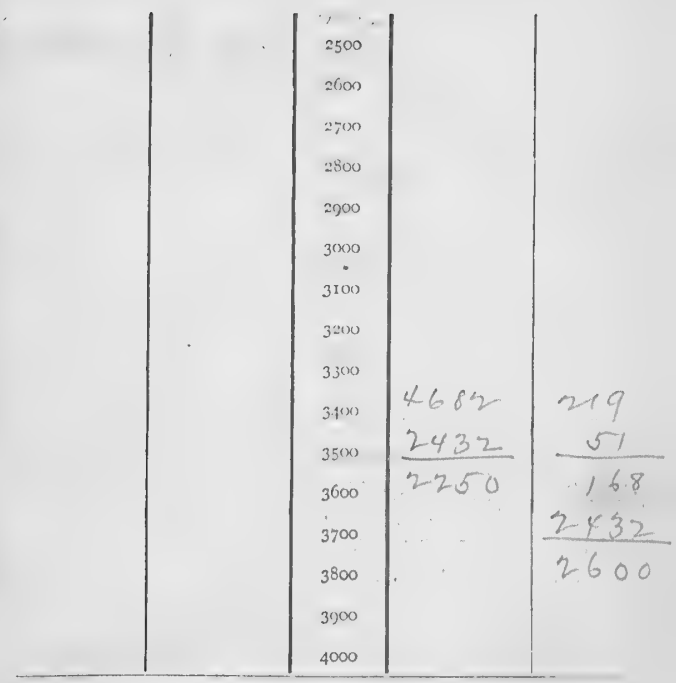

SERIAL TEMPERATURES:

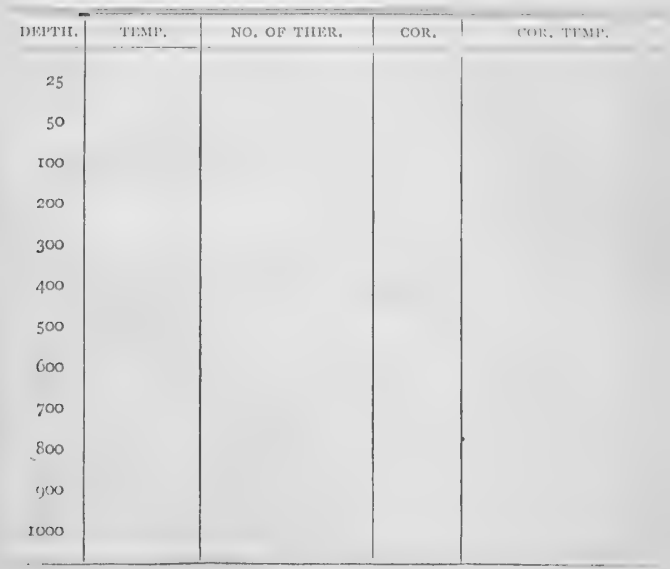

REMARKS : 


\section{(6) 2897}

No.

Dat.e

Liculece Machine." / Reet.. dratr

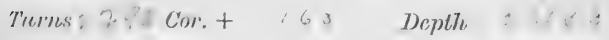

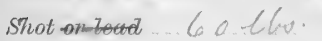

Bottom

Bollom temperature

No. of thermometer.

Cor.

Corrected temperature.

Air ... 7.6. Surface ...76 . Drift

Trawt or dredge

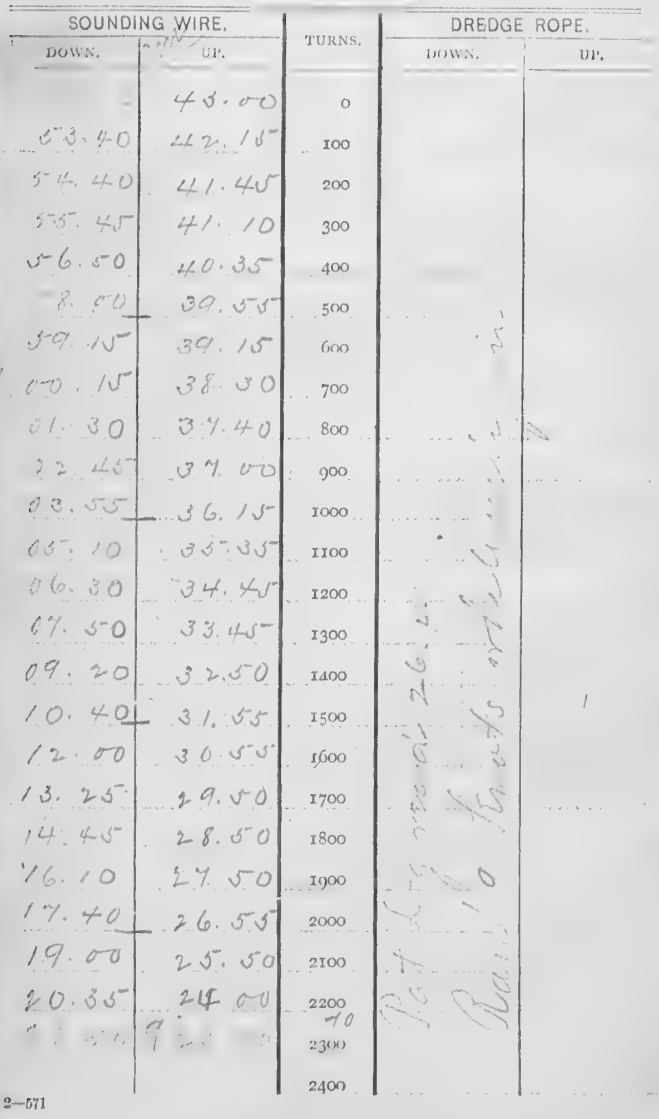




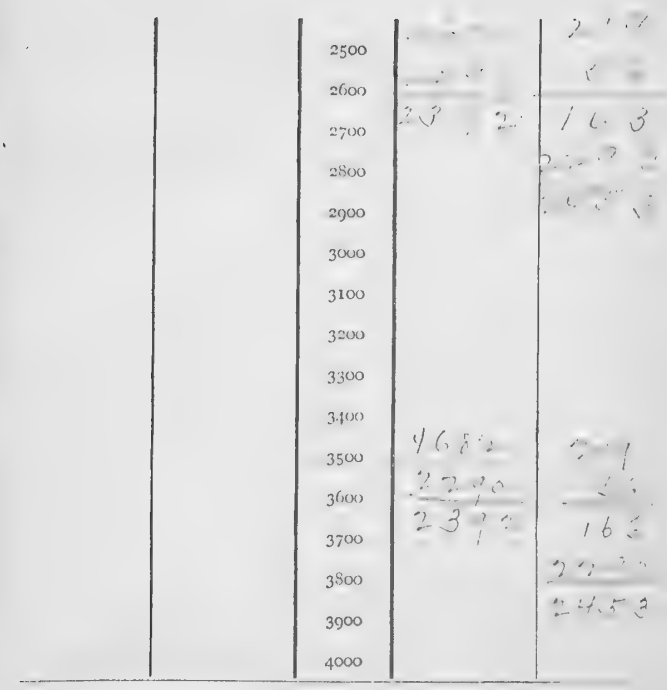

SERIAL TEMPERATURES,

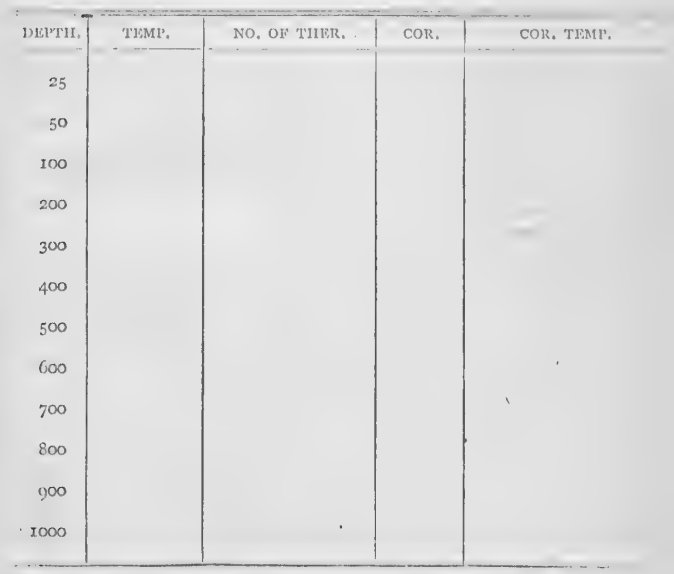

REMARKS : 
trot.

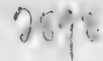

No

Date

Ligebee Machine. */ Reel. Whau

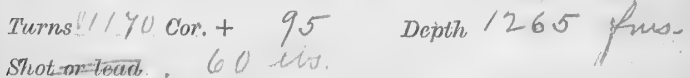

Bottom

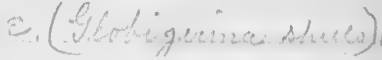

Boltom temperature

No. of therrnometer,

Cor.

Corrected temperature

Air $\quad 76 \quad$ Surface $\quad$ ? $\quad$ Drift

Trawt or dredge

SOUNDING WIRE.

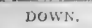

l

2-571
DREDGE ROPF. DOWN. 


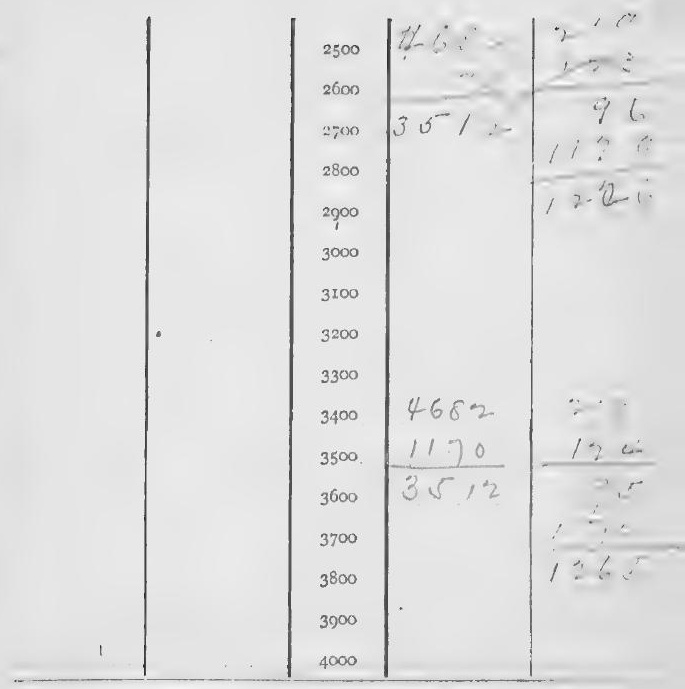

SERIAL TEMPERATURES.

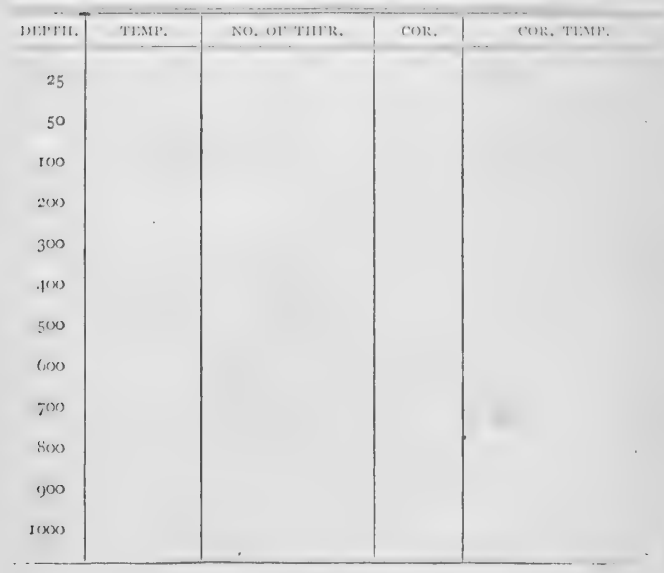

REMARKS : 
501\% Хบें "15แ1:

No. 24.3

Date

Lirolew Machine ' Reel. gläry

Turns 1420 Cor +111 Depth $15-31$ fine

S7iol or-troril

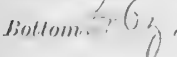

Bottom temperature 35,7

No. of thermometer $5^{-} 48 / \mathrm{J}^{-}$Cor. $-6,3$

Corrected temperature 35.4

Air $\quad 76$.... Surface 76 .... Drift -

Trawt or aredse -

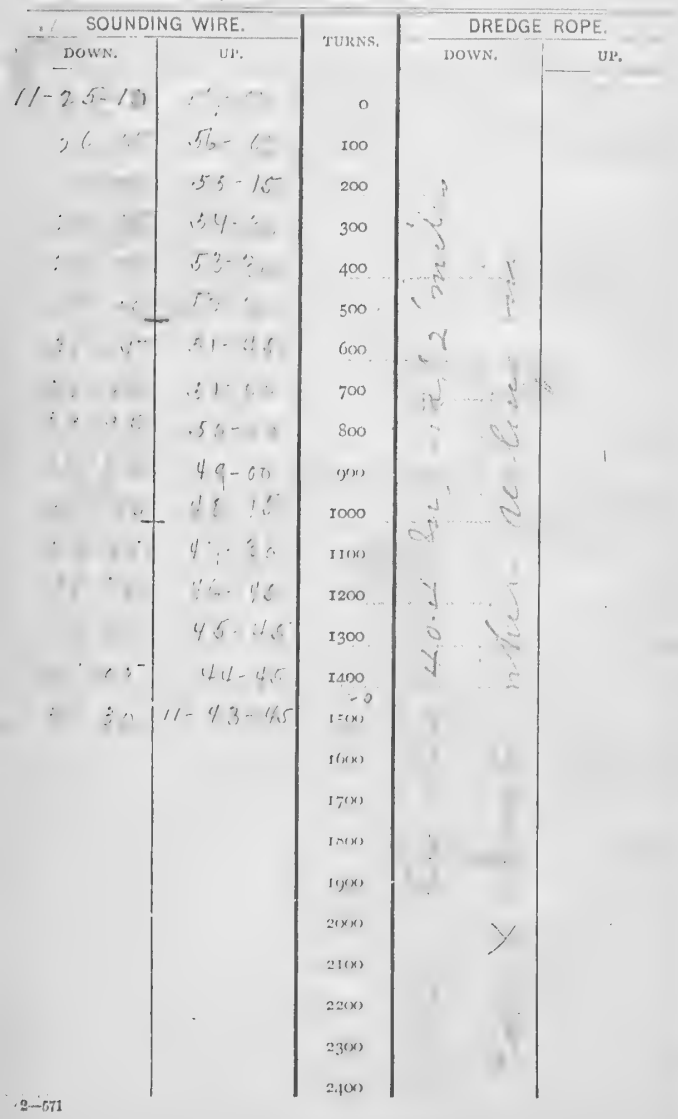




$\mid$

SERIAI, THAIPEATURIS.

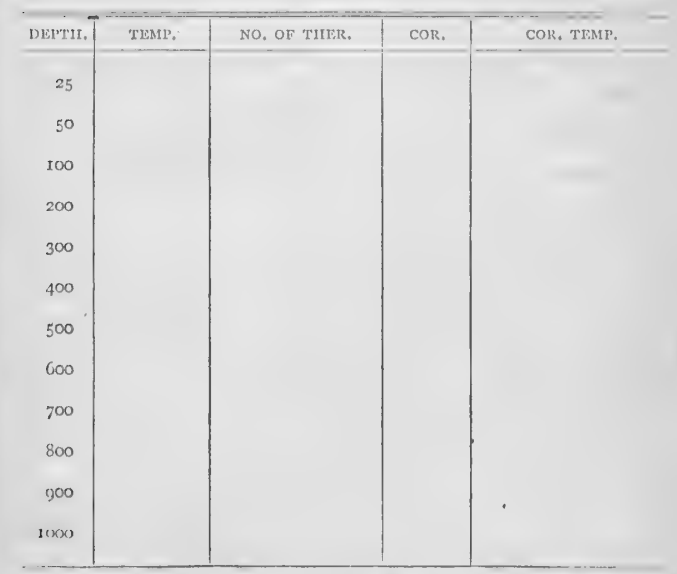

RLMARKS : 


\section{0}

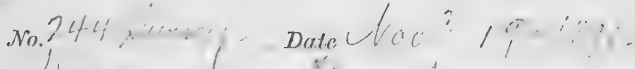

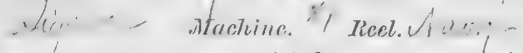

Turius $1.54-4 \mathrm{Cor}+119$ Dopth 1662 (....

Shot-on-teand 6.0 tho-

\section{Diotilom,}

\section{Bottom temperature}

No. of thermometer

Cor.

Corrected temperature

Air

7.7. . Surface

.76

Drift -

Trawl or Aredge

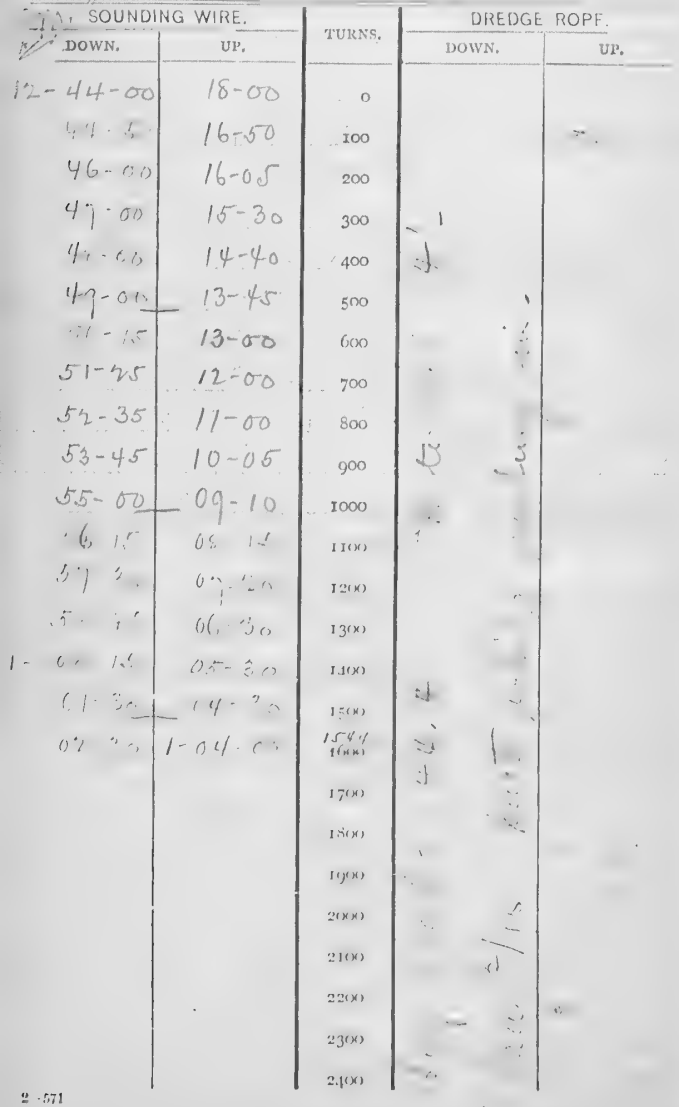




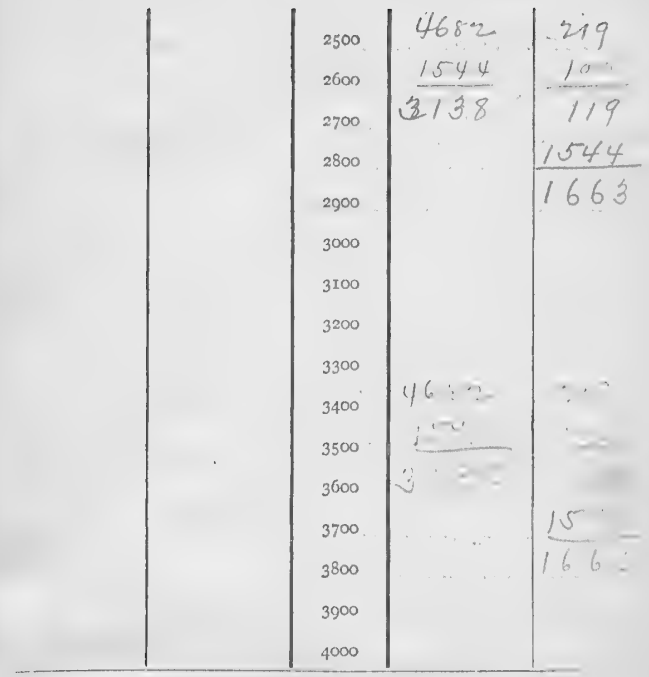

SERIAL TEMPERATURES.

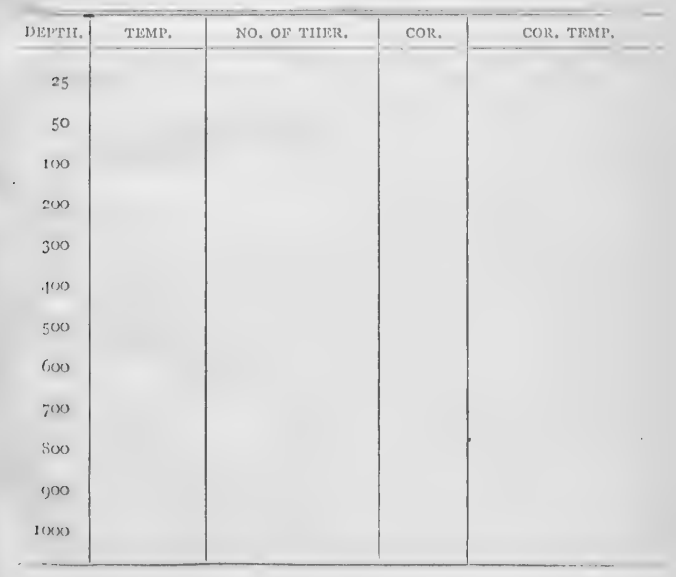

REMARKS : 


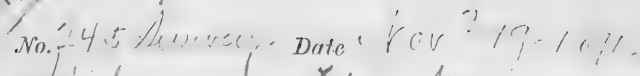
- Jiaplee Machine X / Reet. Sary Turns $2338 \mathrm{Cor}+164$ Depth/25: shot motariat $60 \mathrm{tb} 5$.

Bottom in. of

\section{Bottom temperature}

No. of thermometer

Cor. -

\section{Corrected temperature}

Air Surface

Drift

Trawl or dredgie

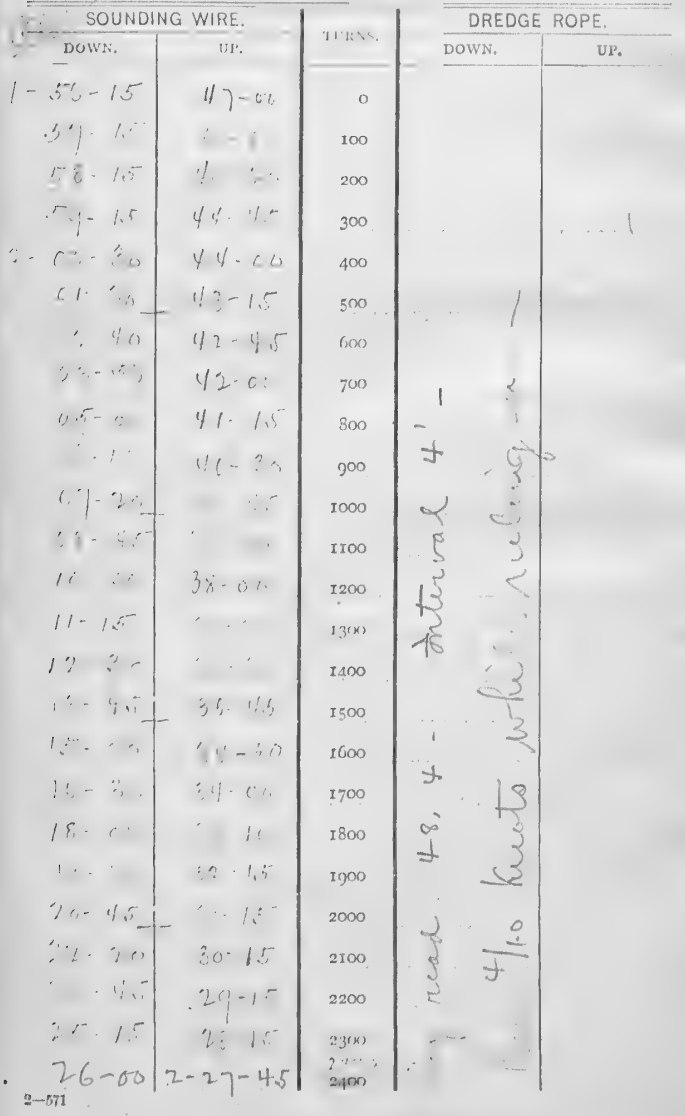




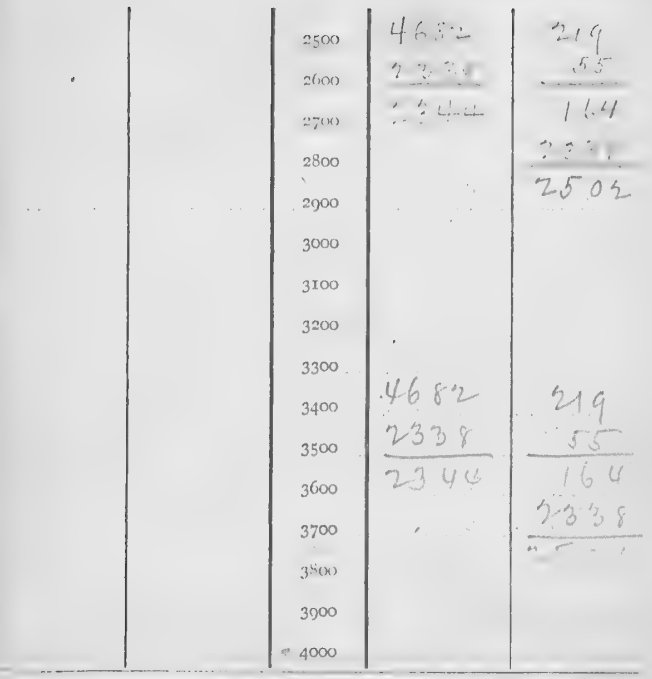

SERIAL TEMPERATURES,

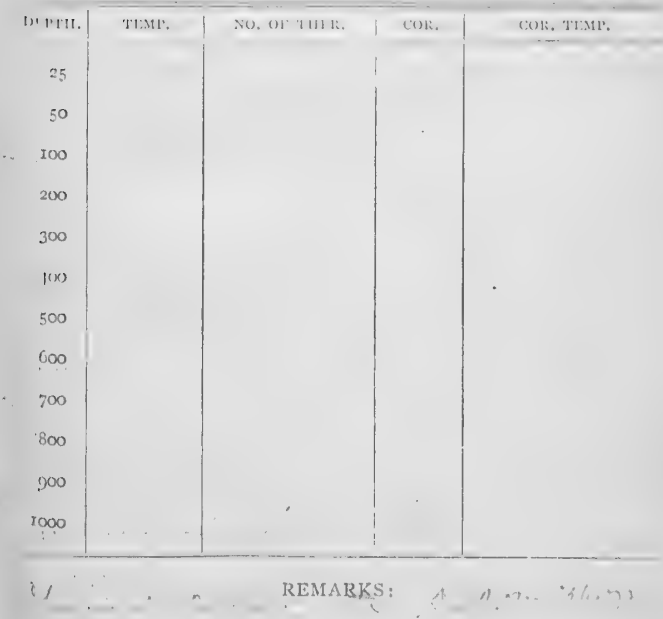


No.

\section{(W67) Date}

Machine. Reel.

I'urne' 8 Coryt Is 5 Depth/783

Shot or lead

Bottom

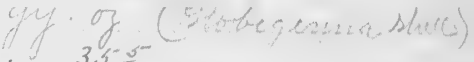

1.- Inottom temperature

No. of thormometer

$$
\text { ( ) } 6 \therefore \% \text { Cor. }-.5
$$

Corrected temperature
Air
78
Surface
Drift

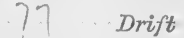

Irawl or Aredge

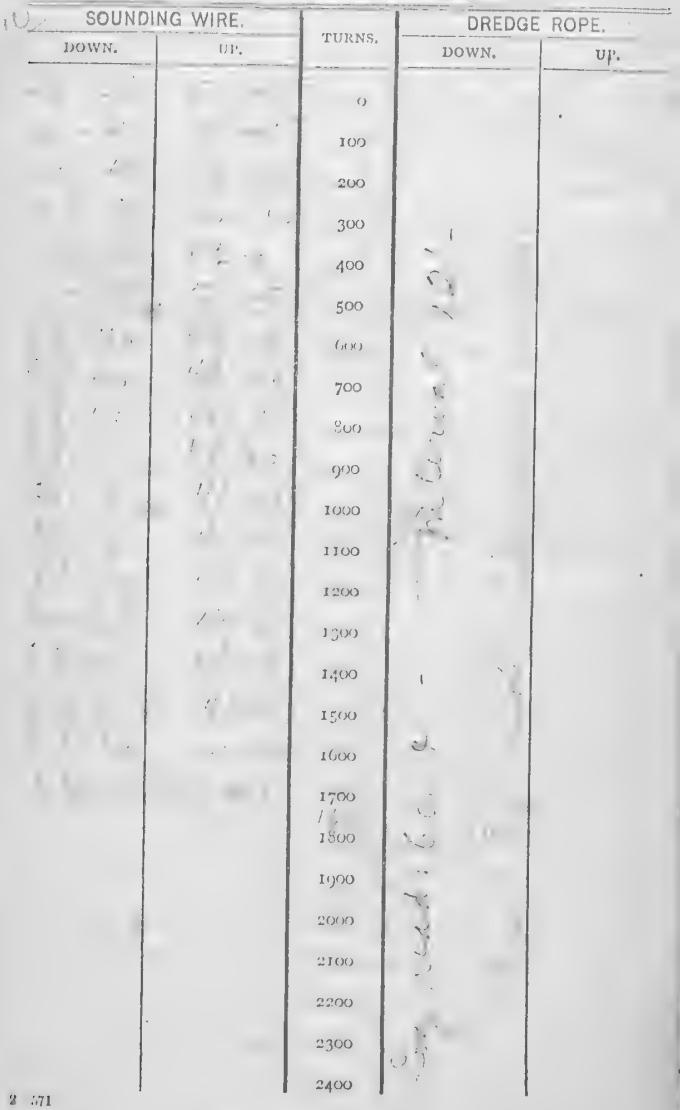

DREDGE ROPE. 


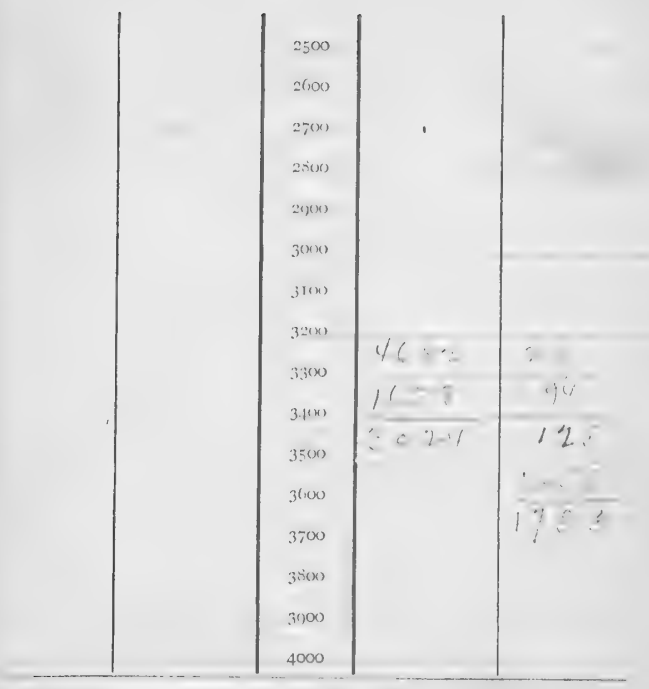

SERIAL TEMPERATURES.

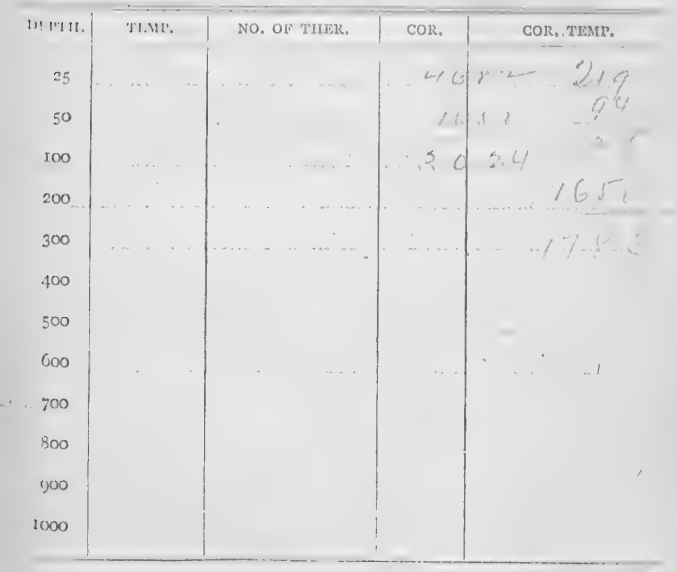

REMARKS : 
No.

\section{Date}

Machine... Reel.
Turns
Cor: +
$<1 .>$
Depth

Shot or-7exd

Botitom

(e) Clas

$$
1:
$$

\section{Bottom temperature}

No. of therrnometer

Cor.

Corrected temperature

Air

Surface

Drift

\section{Trawt or dredge}

\section{- SOUNUING VIRE.}

IUWN.
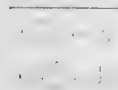

3

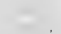

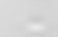

\section{2}




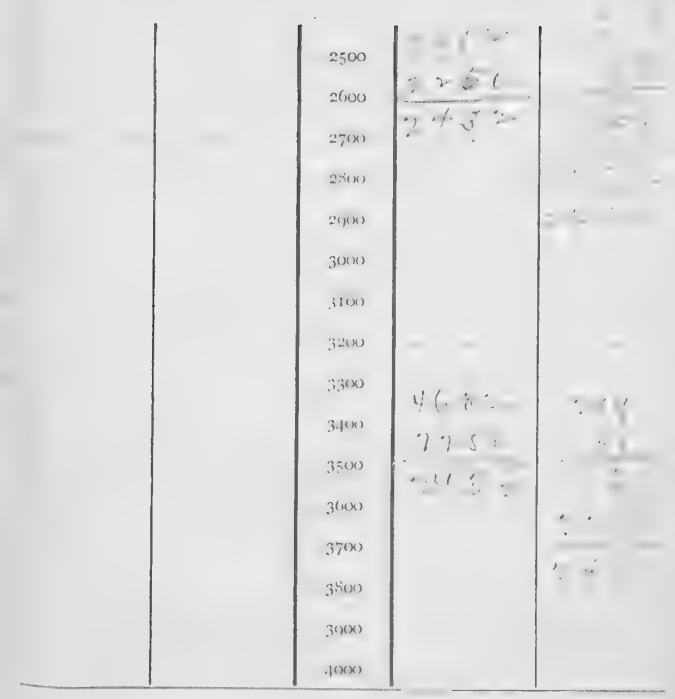

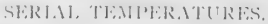

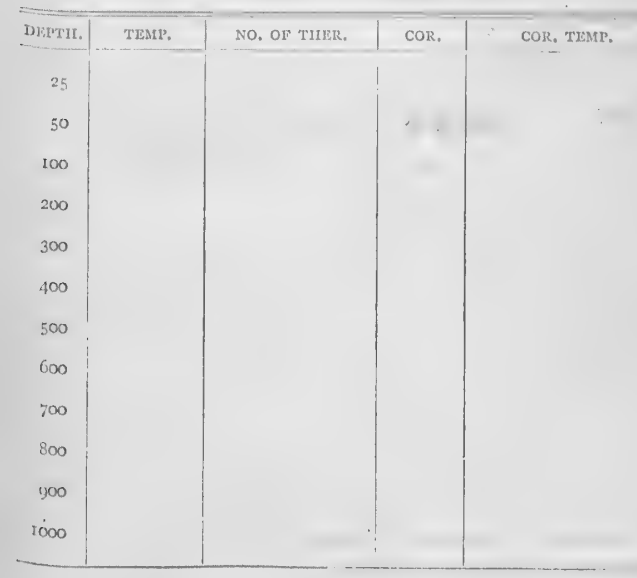

REMARKS : 


\section{$\sqrt{\text { hin. }}$}

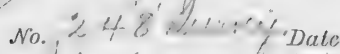

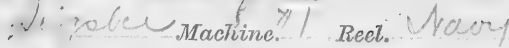

rurves 7301 Cor. +163

Shot ontoad -.60 $66 \mathrm{~s}$

Depth $2464 ! \cdots$

Bottom in , O

Bottom temperature

No. of thermometer.

Cor.

Corrected temperature
Air
sierfiece
Drift

Trawt or dredge

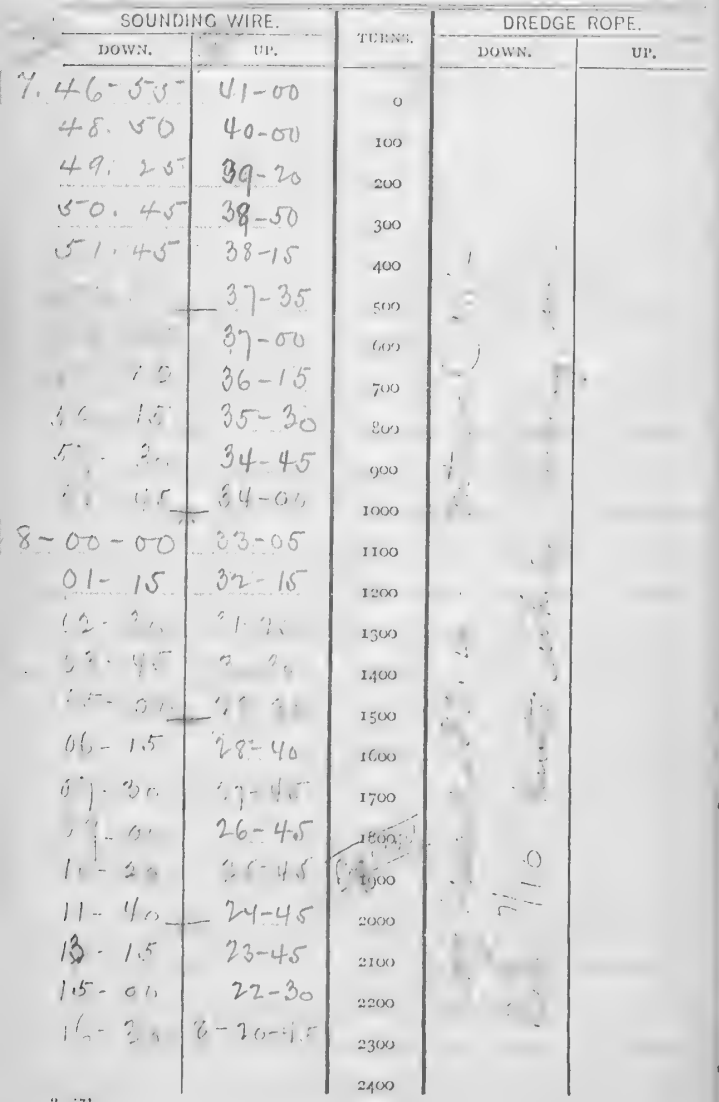




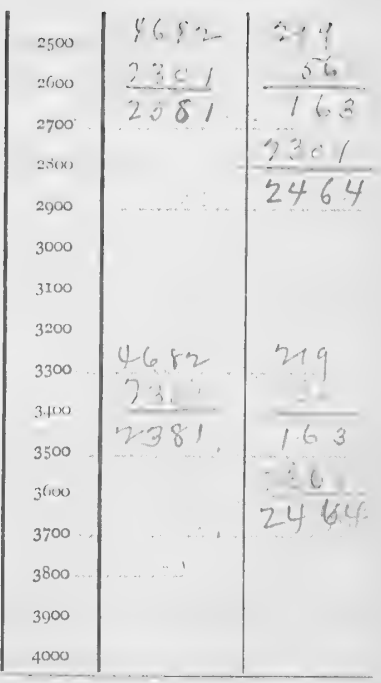

SERIAL TLMPERATURES.

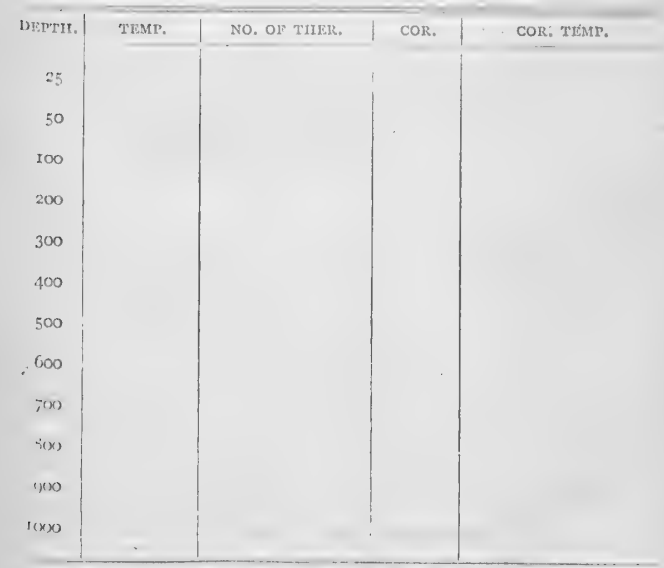

REMARKS: 


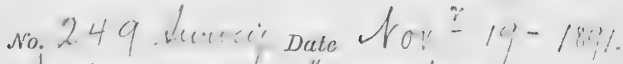

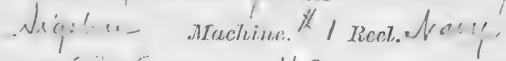

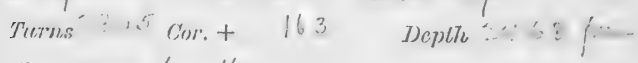

Shot 60 thes

Bottom Yh. Gy, Lava-

Bottom temperature $35 .{ }^{3}$

No. of thermometer 6.6 .665 Cor. $-0,0$.

Corrected temperature 35.3

Air . 77 . Sterface 77

Drift -

Trawl or dredge -

$\therefore$ SOUNDING WIRE. (1i)

$9-49-00$

$i n-r$

$51-00$

$52-00$

$53-00$

$54-\mathrm{CO}_{2}$

$\therefore$ : 3 - ?

$56-00 . \quad 36-30$

$57-15 \quad 35-30$

$58-3.0$

$59-45$

$10-01-00$

$02-15$; 15

$03-30 \quad 30-30$

$04-45 \quad 2 g-35$

$66-00-28-35$

$07-15,27-40$

$08-30$

ir... ? : is

$\because 24-3$.

$13-00 \quad 23-15$

$14-30 \quad 22-00$

$16-00 \quad 20-45$

$17-30 \quad 1 \cdots 19-20$

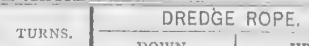

100

200

300

400

500

$(x)$

700

$80 ;$

g00

1000

1100

1200

1500

1.100

1500

$t(6 x)$

I 700

I 800

Ir)o

$200 x$

nIos

2200

2306

2400
UP. 


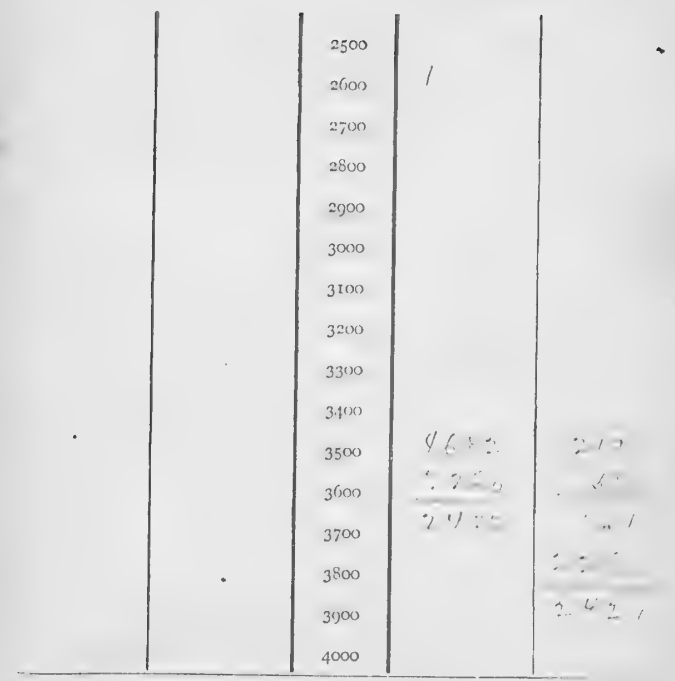

SERIAL TEMPERATURES.

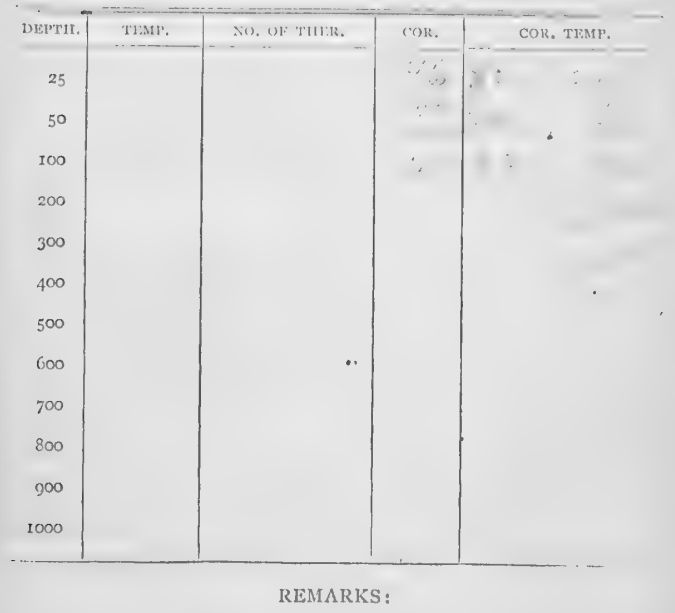


rot.

. No.

Date

Mrachine. Ireel.

\section{Turves}

Cor. +

Shot or 7ead

$\cos x^{2}$

Depth

Bottom. In, G

Bottom temperature

No. of thermometer

Cor.

Corrected temperature

Air 75 Surface 76 Drift-

Trawt or aredge -

\& SOUNDING WIRE.

DOWN.

UI.

$\therefore$

$$
\text { , }
$$

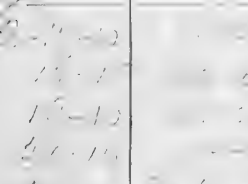$$
\therefore,-1,5,-1 .
$$$$
\therefore \quad-1,0=
$$

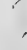$$
\text { , }
$$$$
\cdot
$$

\begin{tabular}{c|c|c} 
TURNS. & $\overline{\text { DREDGE ROPE. }}$ \\
\hline DOWN. & Un. \\
\hline & &
\end{tabular}




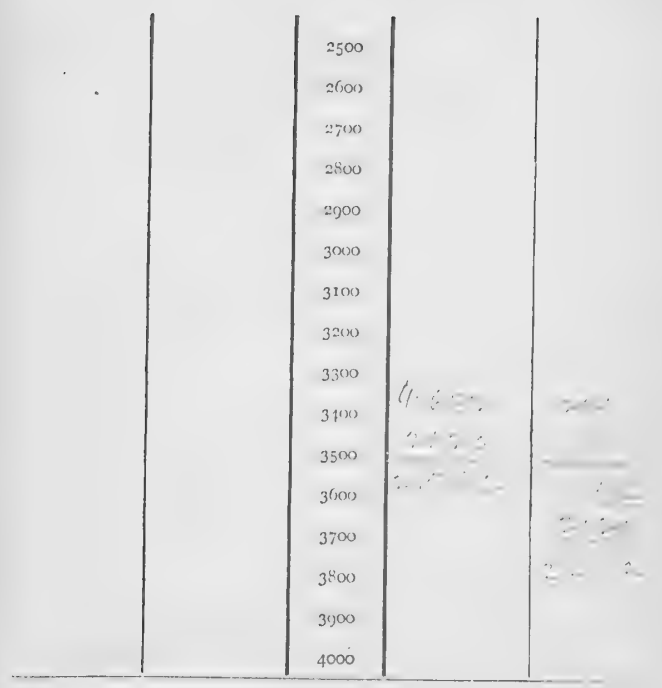

SERIAL TEMPERATURES.

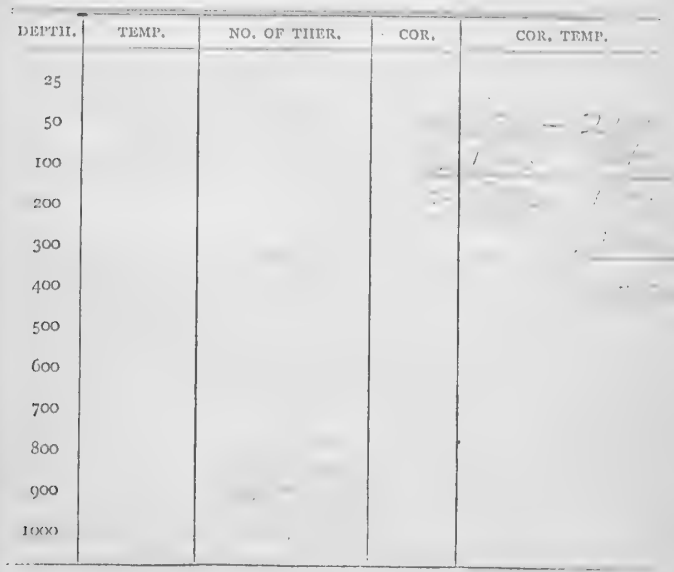

REMARKS : 
Vo.

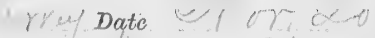

5010 Machine. / Reet. Turns 2185 Cor +156 Depth 23441

Shot orizared (ov) $\ell$

Bottom ... $\mathrm{N}, \mathrm{O}$,

Bollom temperature

.ii). of thermonmeter

Cor.

Correceted temperature?

Air 75 . Surface 76 Drift -

Trawl or dredge -

$\rightarrow+$ SOUNDING WIRE.

$\rightarrow$ DOWN.

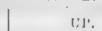

$-1 i$

\begin{tabular}{c|c|}
$\because, \quad \ldots$ & \\
$\therefore-\ldots$, & $\cdots$,
\end{tabular}

,

$1 \div i=$

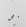

$2+-25$

$25-35$

$29-50$

$31-10$

$8 n-20$

$33 \times 40$

i) -00

, 1 -

23.

310

$30-00$

if $0-25$

$-11-35$
III.

20 43

(5) $3-40$

$62-35$

$5+25$

$50-15$

$49-15$

L.t $8-15$

$$
\text { il }
$$

$46-32$

$1+5=30$

L. 61.4.

$413+16$

$\sqrt{1}$

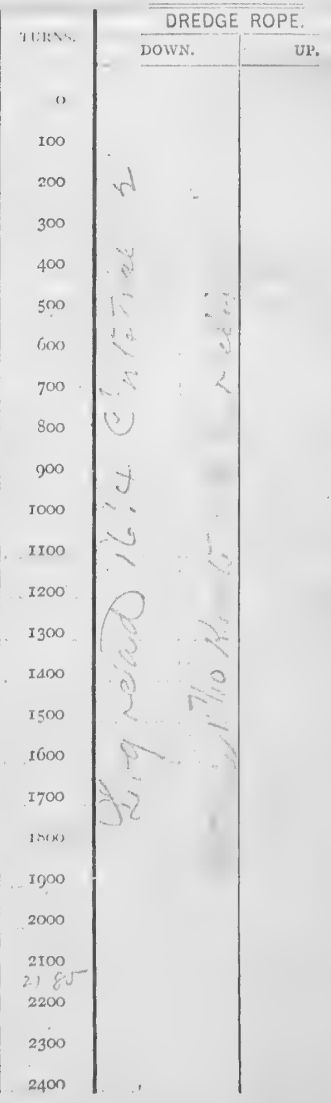




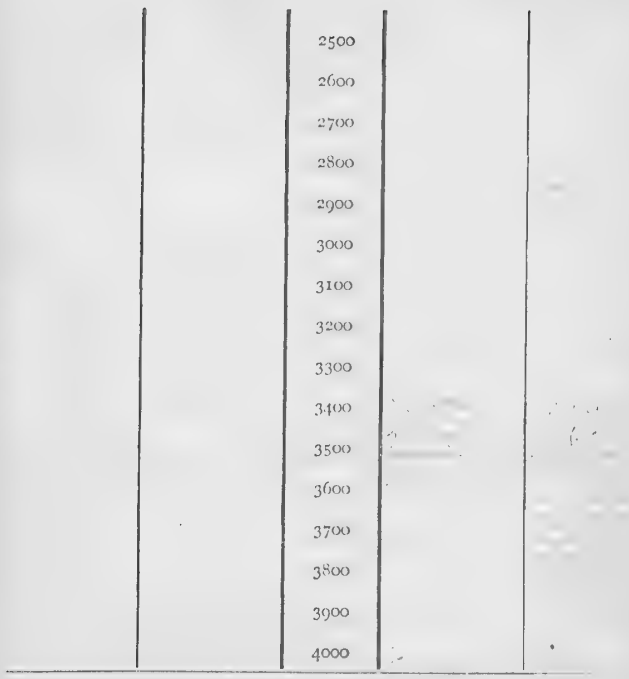

SERIAL. TEMPERATURES.

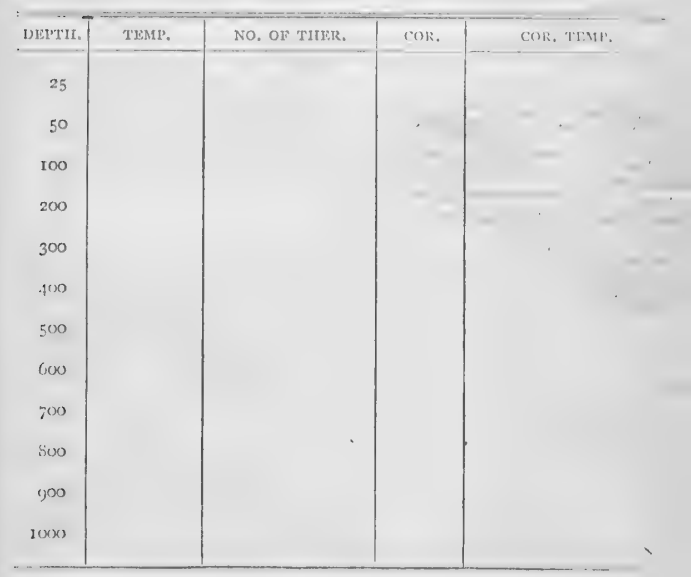

REMARKS : 
500. 9149

No. 53 A curey Date fir

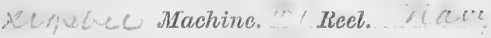

Turnsw24 Cor. +160. Depth 2408 Hew

Shot ar Iacad 6.0.6hs

Bottom in: Gy,

Bottom temperature

No. of thermometer.

Cor.

Corrected temperature

Air 75 Surface …76 Drift

Trawt or dredse

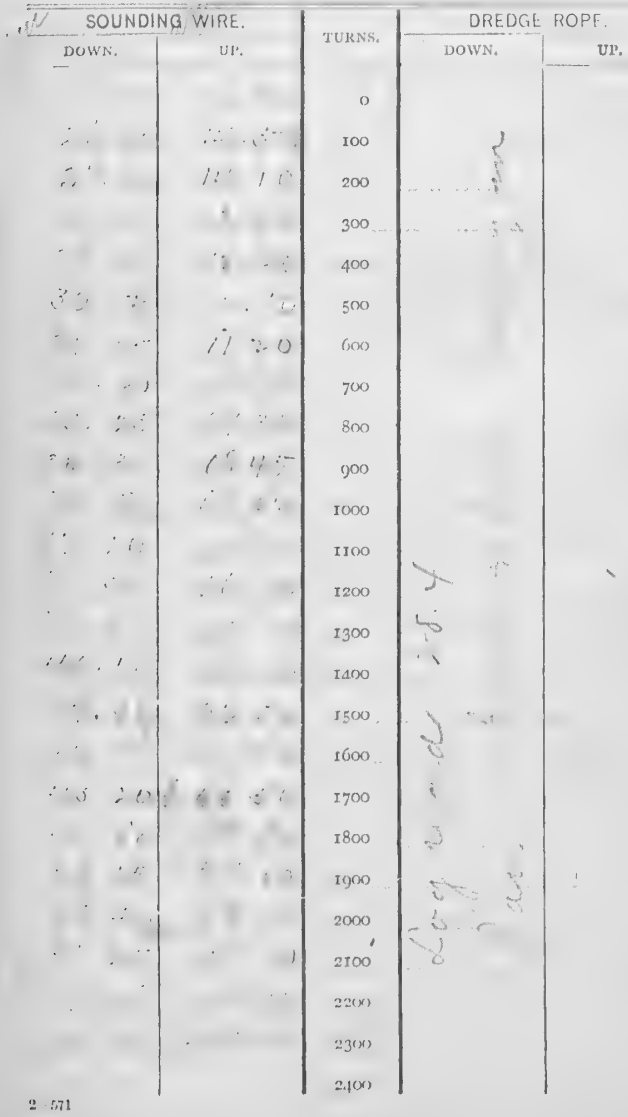




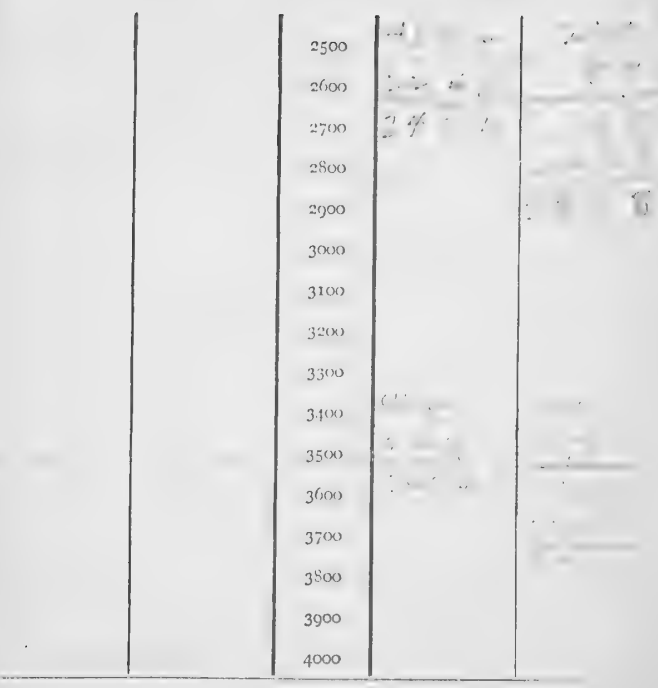

SERIAL TUMPERATURES.

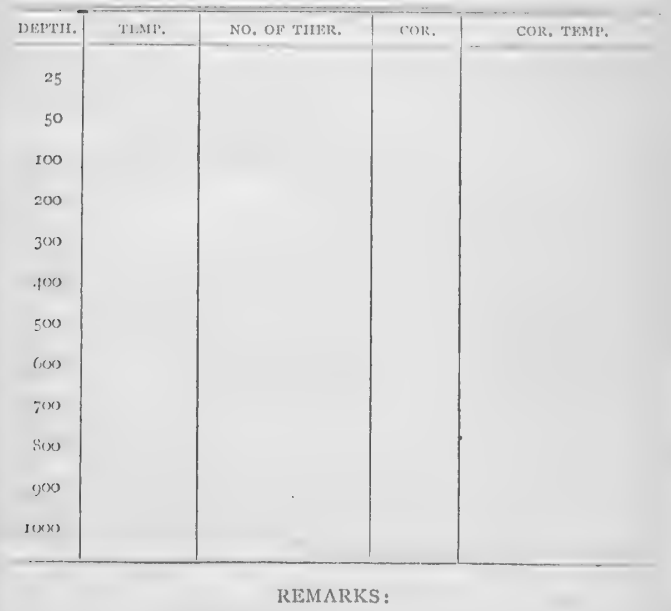




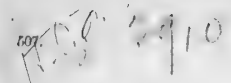

No. 254 Severi, Date Chomen 20 1. Machine. / Reel.
Turns 2265 Cor +161
Depth 2426

snot on toad $60-669$

siotum, lon, on,

Bottom temperature

No. of thermometer

Cor.

Corrected temperature

Mir. 7 Surface 76. Mrift

Trawt or dredse

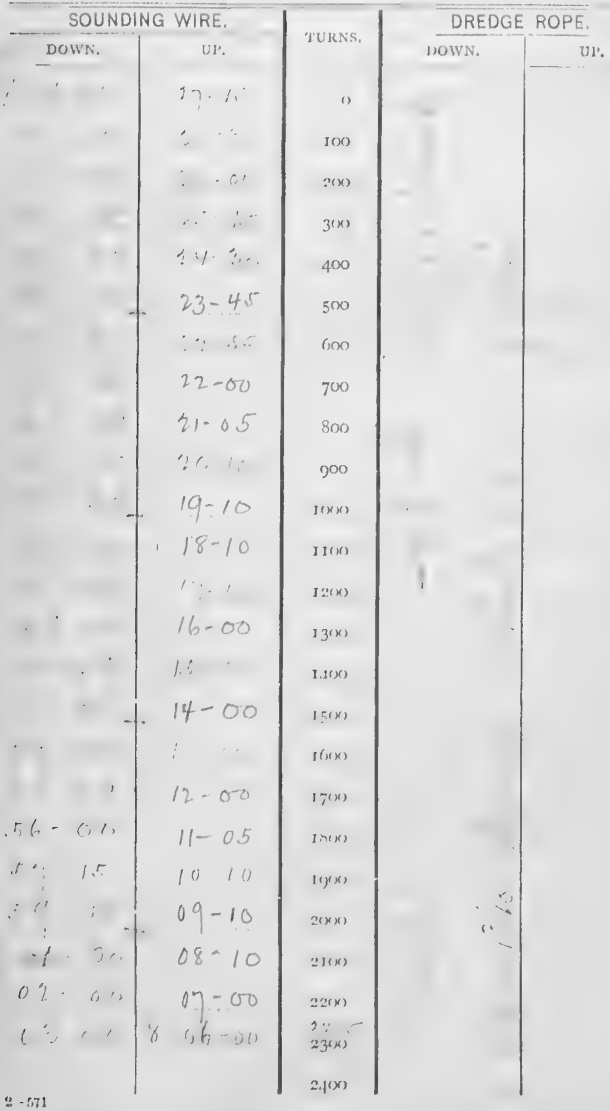




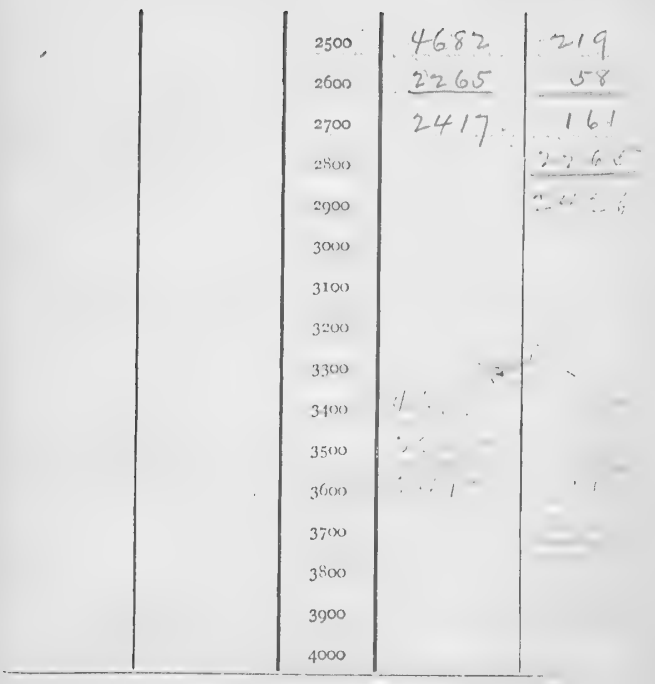

SERIAI. TISAUERATURES.

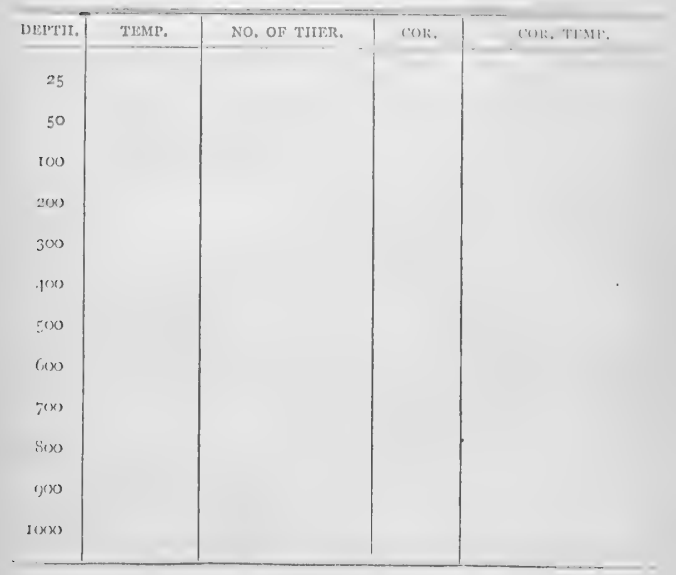

REMARKS : 


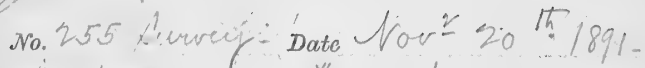
A...... Machine. "*eel tram

Turns 2305 Cor. +163 Depth 2468 fruoshot or-tead 60 this .

Bottom thiog.

Bottom temperature $3,5,4$

No. of thermometer 6.6 .665 Cor. -

Corrected temperature 35.4

Air is Surface. Th Drift -

Trawl or dredge -

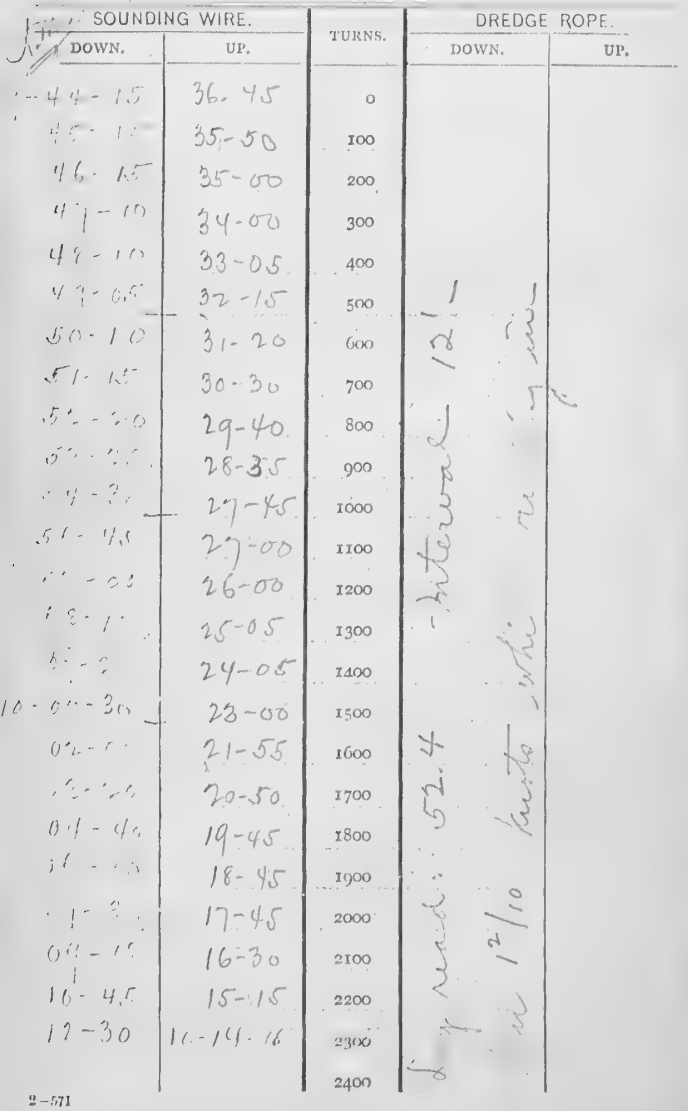




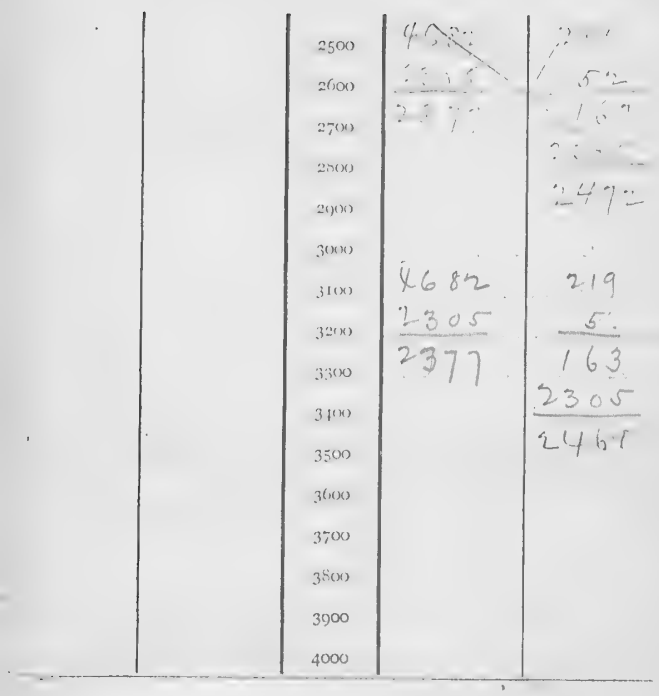

SERIAL TEMIPERATURES.

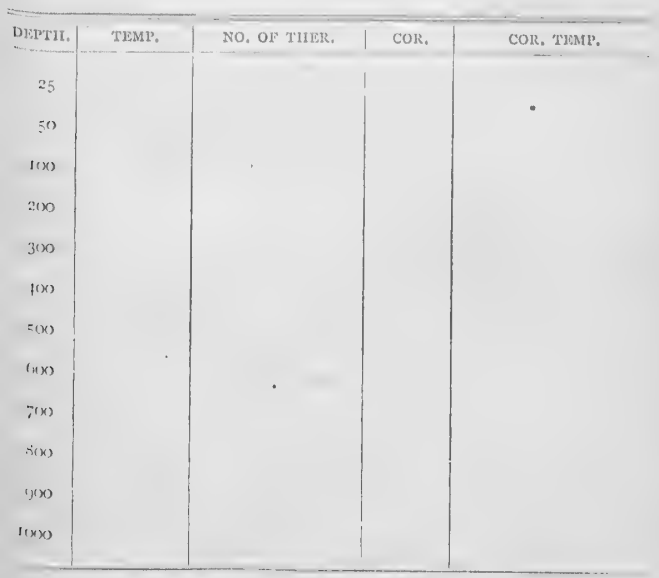

REMARKS: 


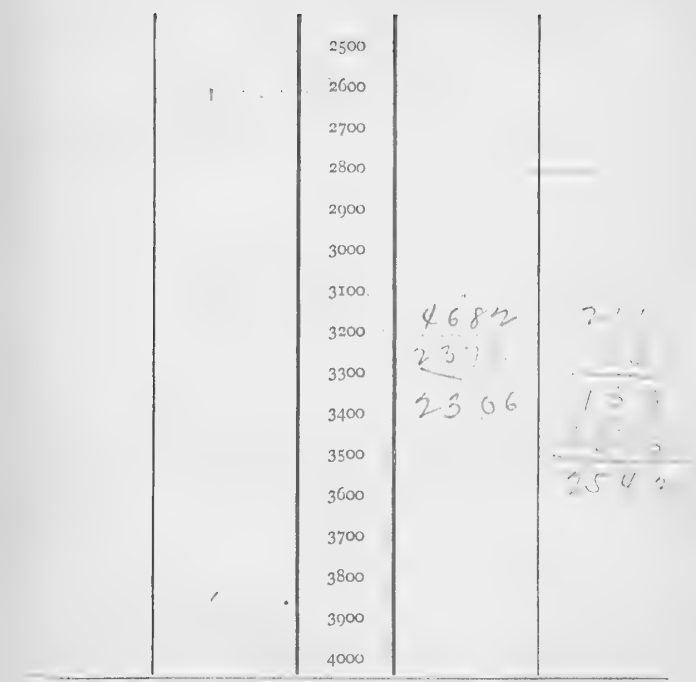

SERIAL TEMPIRATURES.

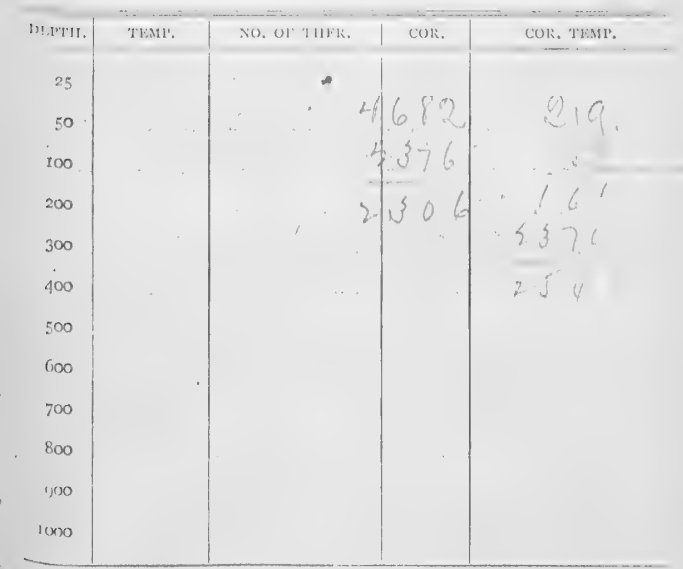

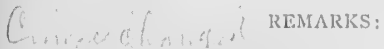

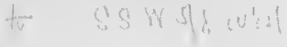


Turus $\quad / / \mathrm{Cor}_{0}+\quad \therefore$ DoplT

shot or bead .... Co Cbs

Bolitom Qfin :ne $s-4$

Bottom temperature

No. of thermometer

Corrected temperature 35,4
Air
77
Surface
7)
7)rift

Irawl or Aredge

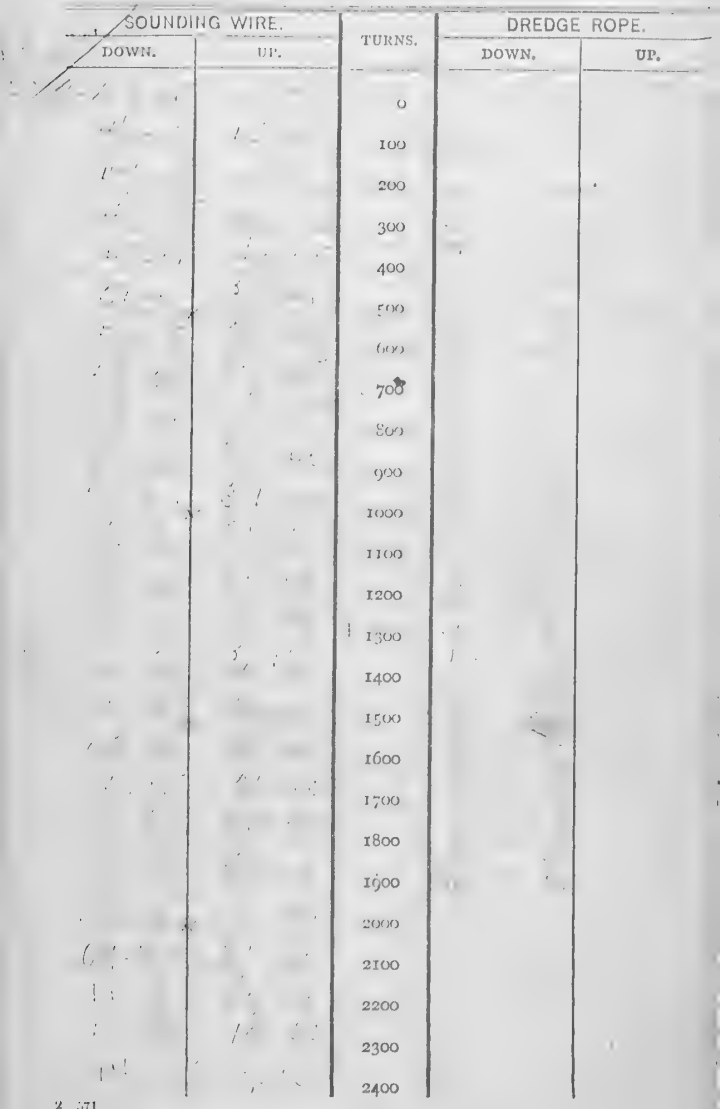




.

SERIAL TEMI'ERATURES.

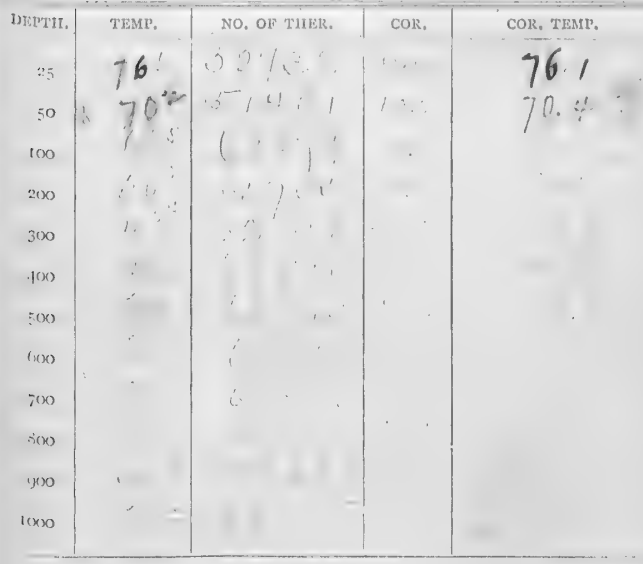

REMARKS : 
i) 6

No. 258 tratectio 20 'unpue Machine N Reel. Jlais

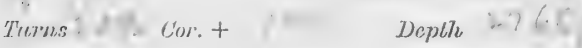

Shot or-lead . $6 t$

Bottom $\mathrm{ln}, \mathrm{M}$.

Bottom temperature

No. of thormometer

Cor.

Corrected temperature
Air . . 78
Surface
77
Drift

Trawt or dredge

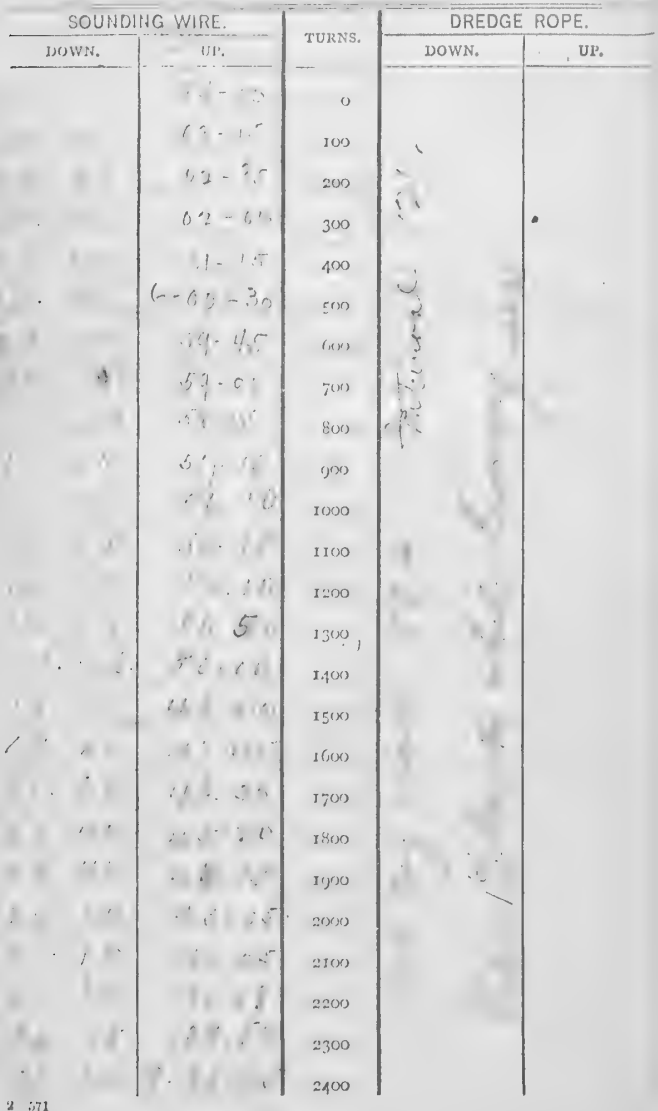




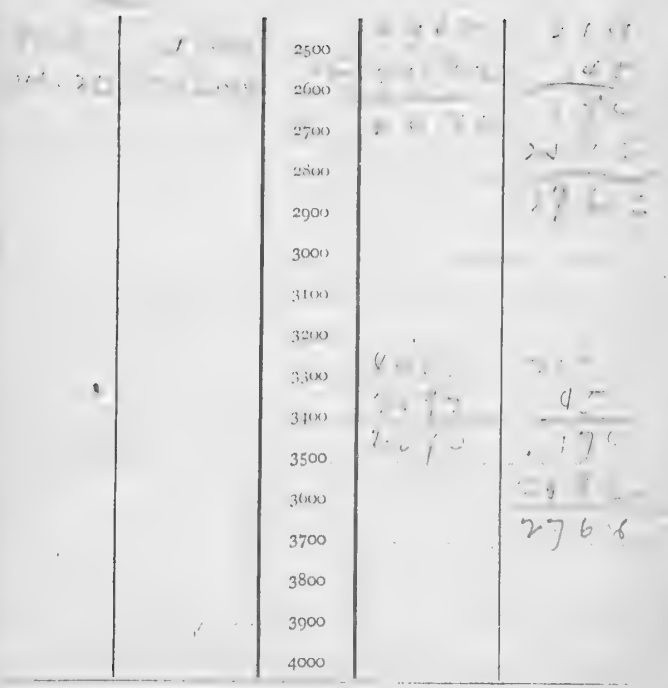

SERIAL TEMPERATURES.

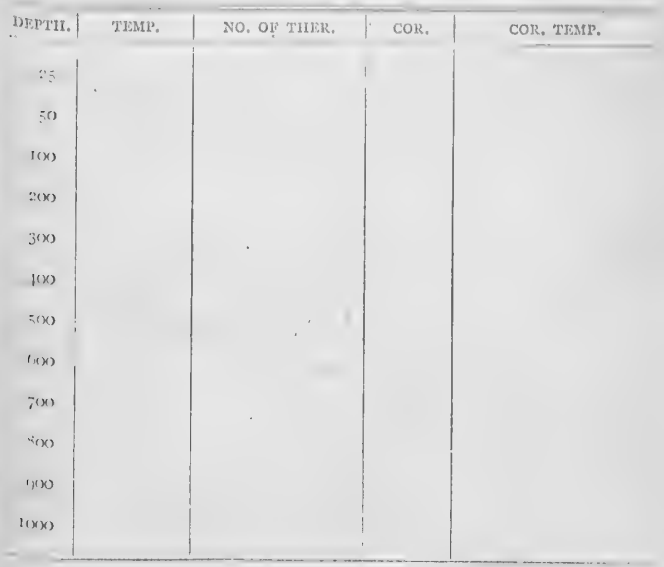

REMARKS : 
1.ing.

No.2.5a Shemest, Date Hrav? 20.-181.

diquber machine \&/ Rect. Hanj -

THT)

Cor. +

Deplth

Shot $60 \mathrm{ttos}$

Bottom for e

Bottom temperature

No. of themometer

Cor. -

Corrected temperature

Air 7

Surface

Drift

Trawt or dredge-

- I SOUNDING WIRE.

$\int_{1} 19$

, Jow:

$26-? 0$

2 3. 3.

: i. 1.5

$\therefore,-10$

$31-15$

$\because \cdots$,

, '.

$\because 15$

$3 ;, 1$

$j \div-1 ;$

$\therefore 2-$.

i. is

i

i; 1.

4)

$4: \cdots-$ Ii. ruRns

DREDGE ROPE. UP.

$115-6$

$46-2$

1) : .

$\because \therefore-\because$

bi i i .

$5 \cdot \therefore$

$\because \because \cdot A$,

t.r. ㄷ..

$4 \quad 371$

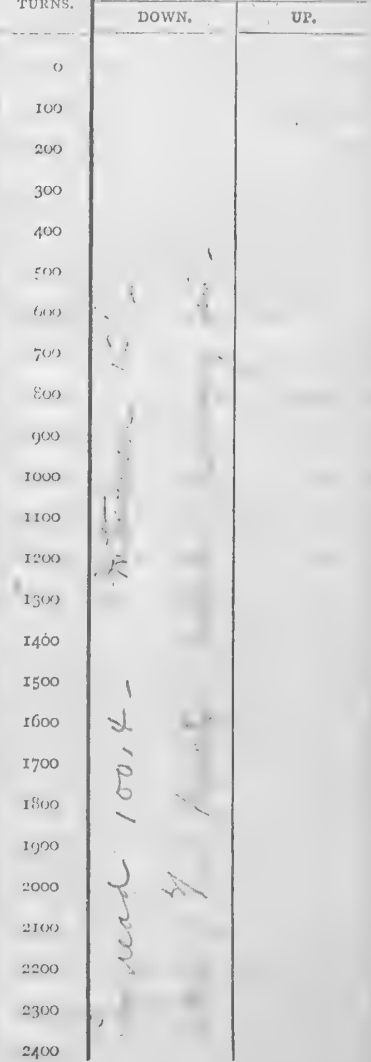




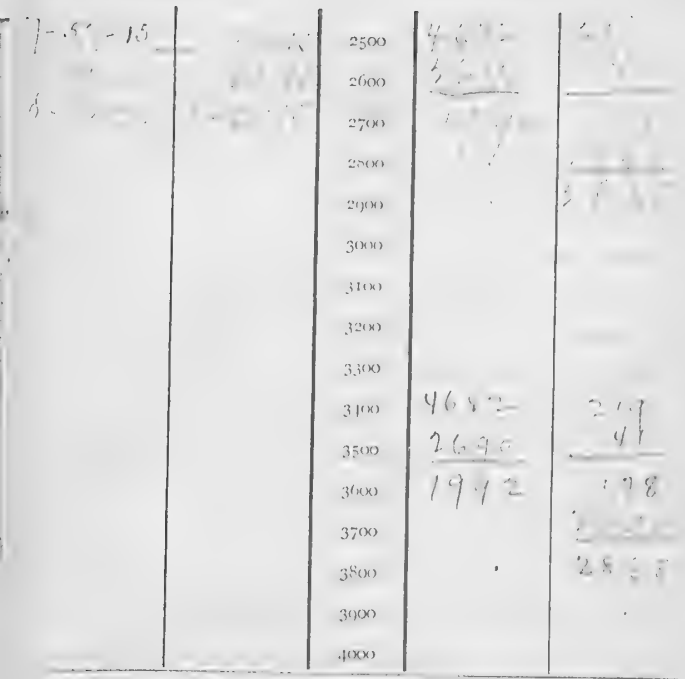

SERIAL TEMPERATURES.

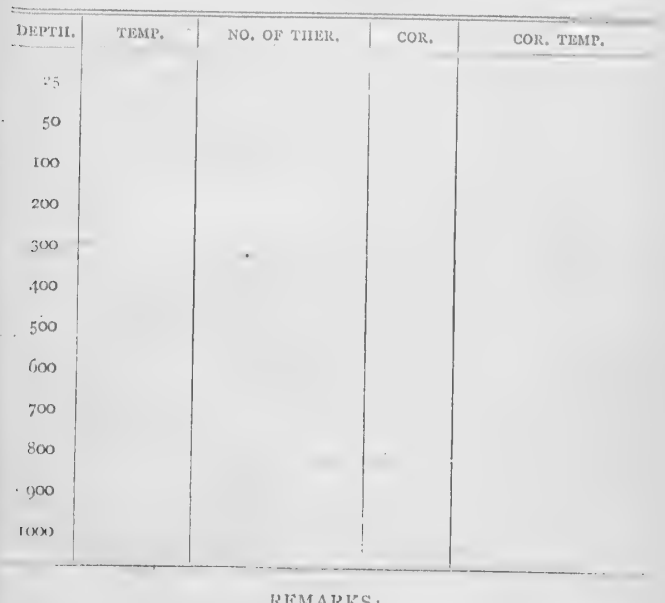

REMARKS:

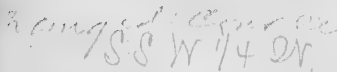


$\therefore 260$

\section{Date}

Machine, Reol. Wh rery...

Turns 269lor. 179 Depth $28 \% 8$

Shot or lead

Bottom. Ffomin

Bottom temperature

Noin thermometer $6666 \sqrt{ }$ Cor. -

35,3

Corrected temperature

Air ..7. . Surface 77 . Drift

Trawt or dredge

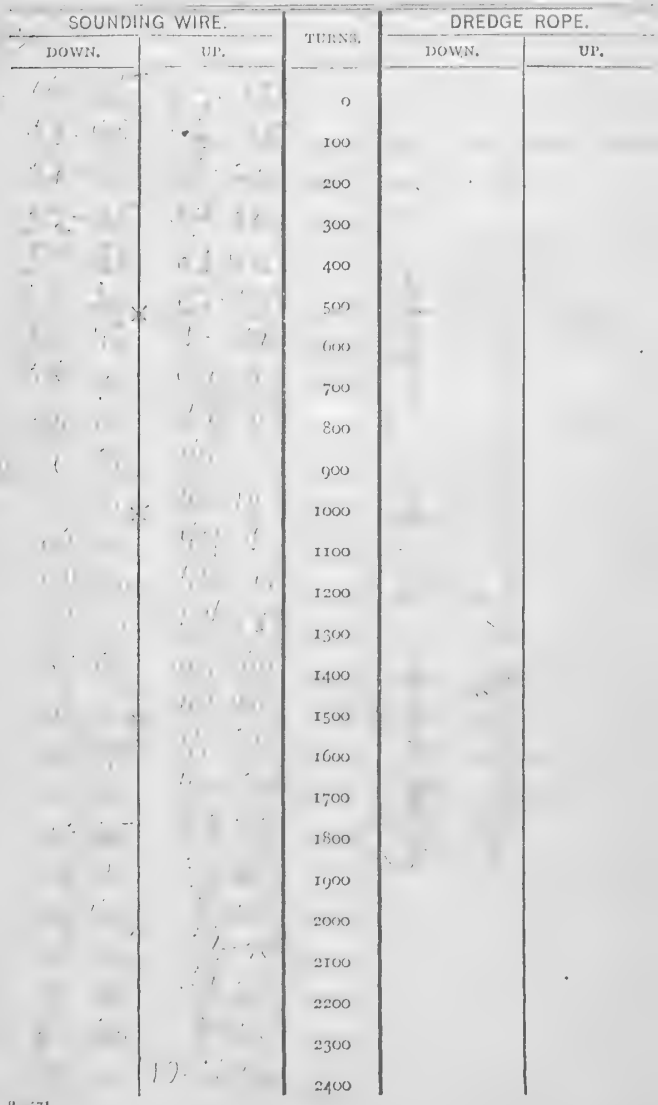


No. 261 Date

\section{Machine. Reel.}

Turns? 446 Cor. +169 Depth 2615 Shot ontoad $\quad 60-i 1$

Botiom $11, \pi$,

Bottom temperature

No. of thermometer

Cor.

Corrected temperature

Air 76. Surface 77. Drift

Trawt or dredge

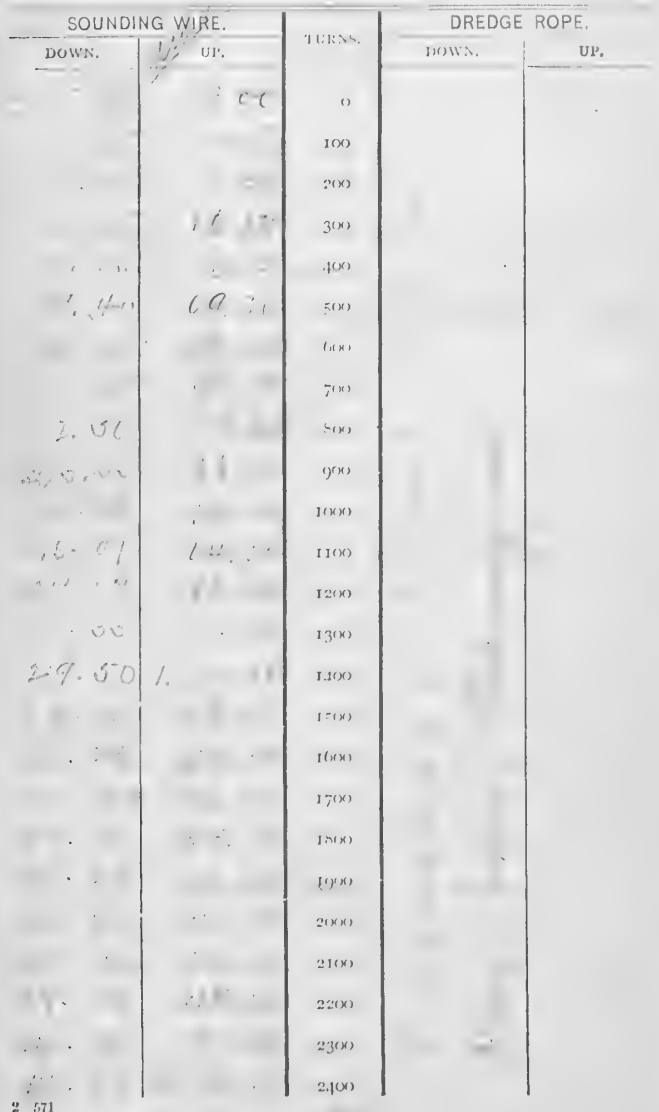




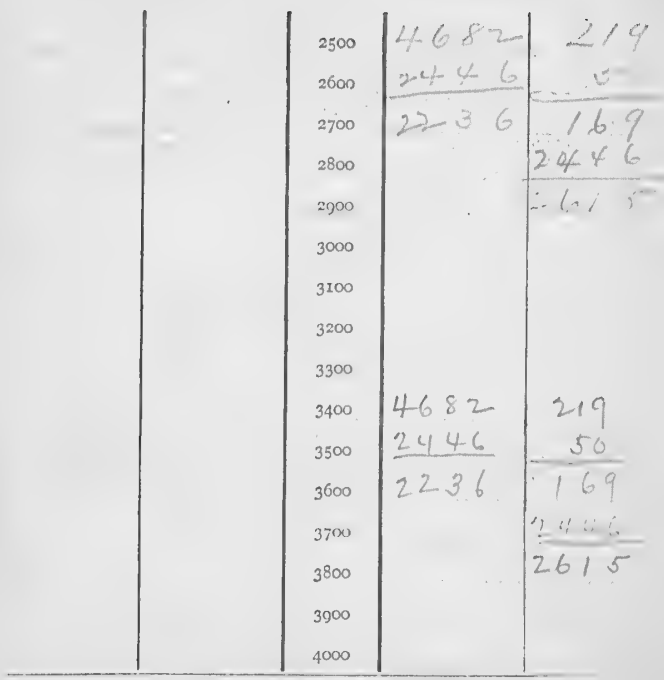

SERIAL TEMPERATURES.

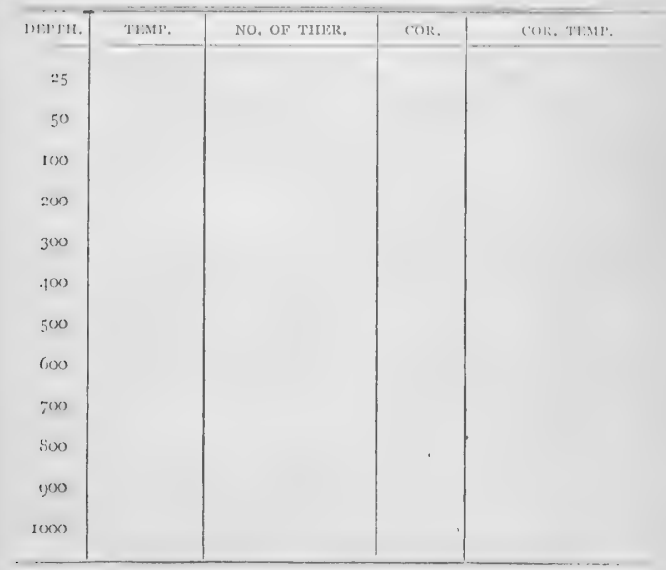

REMARKS : 


\section{vel. 2918}

No.

$\tan 21=$

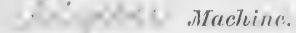

Reel.

Turus: $\quad C o r+1, \quad$ Depth $2-i 76$

shot op zead ' $60 \mathrm{lts}$

Botiom. If in frue s

Bottom temperature

No. of thermometer.

Cor.

Corrected temperature

Air .7 6 . Surface ..7) Drift

Trawl or dredge

SOUNDING WIRE. $\because$ DOWN.

1.26 .00 $\therefore$.

$2 \%, 35$

28.2 .5

34.20

30,2

31.10

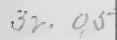

.33 .0 .5

it. 00

$\therefore 5012$

36.20

$3 \% \quad 35$

$1+: \quad: / 8$

40.00

$2.1 .=0$

$42.35=$

$4.3 \cdot 50$

$45=20$

46.40

I! I . ,

49.35

in a ib

i.

ㅁ. 571
NG WIRE.
UI. 21,02 . $=1 . .7$ 19.35 $18 \cdot j^{2} \mathrm{C}$ 18.15 17.35 16,40 16,100 $15=10$ 14,10 13.150 12.10 10. 55 a. 11, 68.35 $0 \% \cdot 20$ $06.05^{\circ}$ 04.45 (3). 33 02.05 $07 \cdot 23$

IgO0, $2(x)$ 2100 $22(x)$ $23(x)$ 2. $f(x)$
DREDGE ROPF (u) W. Ui. 


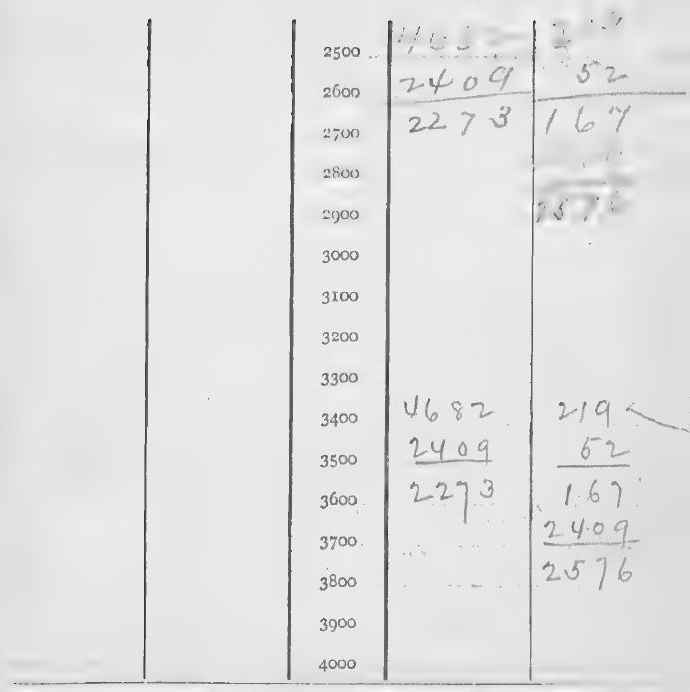

SERIAL TLMPERATURES.

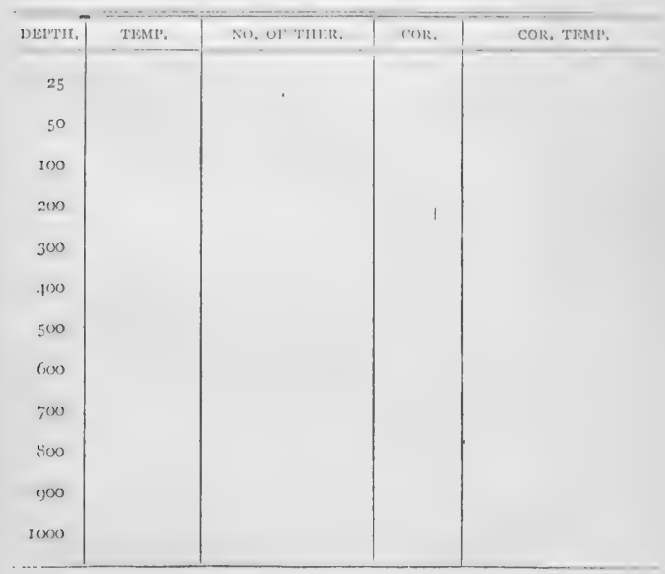

REMARKS : 
1.07.

$\therefore \forall ; \div: 11$

No. 26.3

Date

\section{Mruchine / Reel.}

Trums $191.5 \mathrm{Cor}+141$

J)epth, $2 \therefore \therefore \therefore$

Shot or tead. 6.0 to $\$$.

Bottom 4 in the S

Bottom temperature 35,5

No. of therrnometer : 66665 Cor.

Corrected temperature 35,5

Air 75 Surface 77 Drift -

Irawt or dredge -

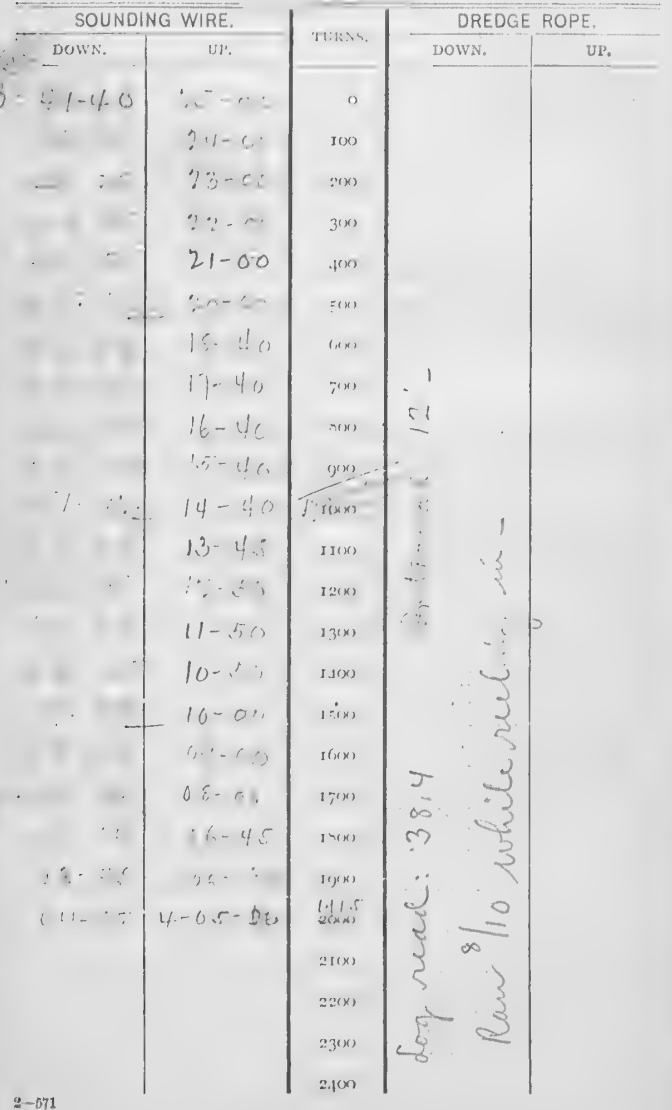




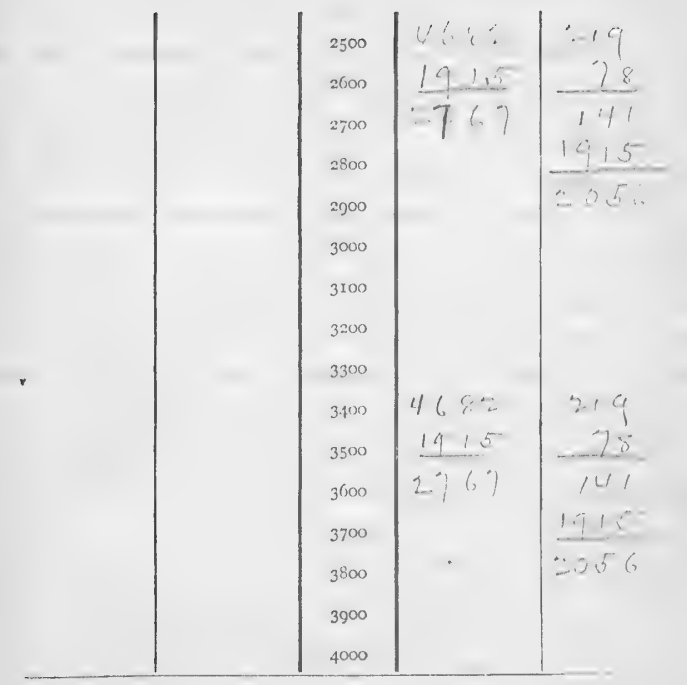

SERIAL TIMPERATURES.

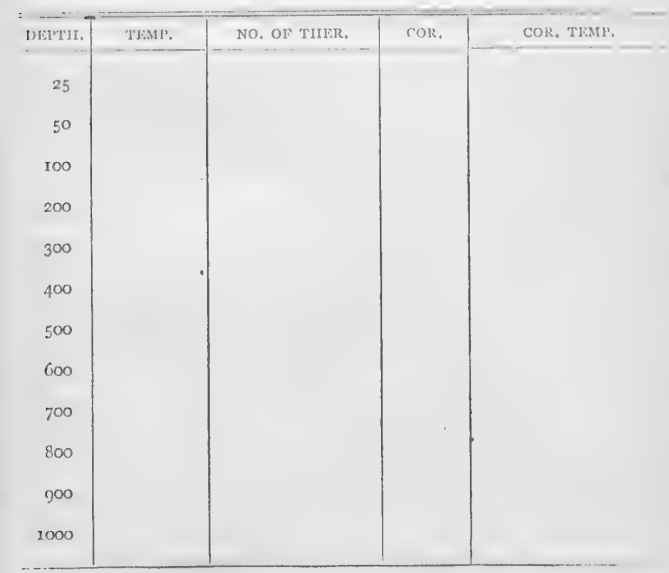

REMARKS: 


\section{0 \\ $26 \mathrm{P}_{i}$}

. $Y$ o. Sia.hee Machine. *I Reel.Srary-

Turrus 524 Cor.t 46 - Depth $5 \%$.

shot ardead $60 \mathrm{tb} 5$.

Bottom if nu tre $s$

Bottom temperature

No. of thermometer

Cor. -

Corrected temperature -

Air ..76. Surface 7\% Drift -

Trawt or dredge -

NOI SOUNDING WIRE.

S DOWN. WF.

$\therefore$

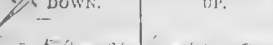

$+5-c+\cdots-i c=-2$

$=, 54-3 \%$

$.5 \div-01 \quad 58-1, \ldots$

$58-15$

$\therefore$ $\because \because-\because \because{ }^{\prime} \quad \therefore$

\section{I}

Turns:

$n(x)$

$3(x)$

$f(x)$

$5(x)$

$\therefore 6$

(A)

$7(x)$

$\therefore(x)$

(j)

Jor)

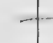

$2-571$
DREDGE ROPE.

DOWN.

Ux. 


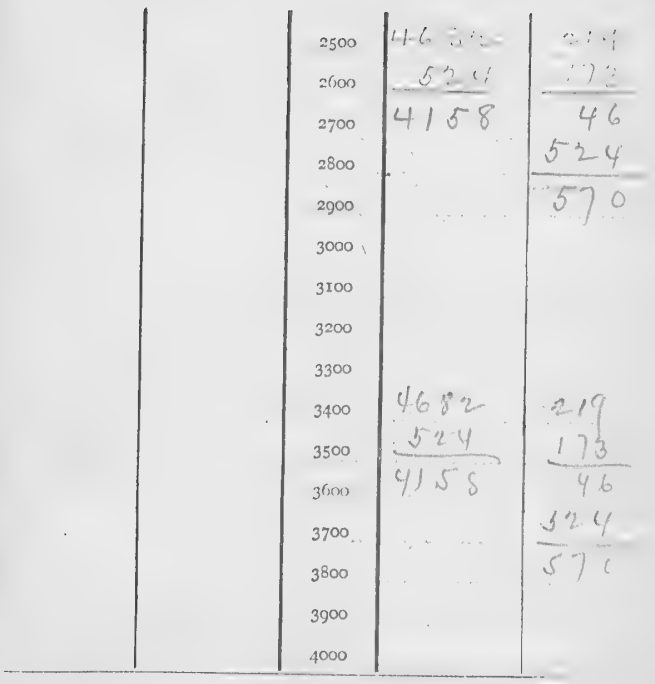

SERIAL TIEMPERATURES.

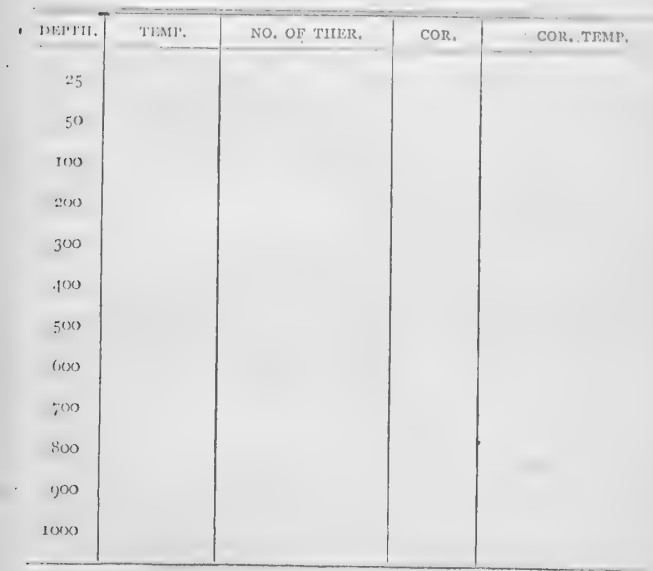

REMARKS : 


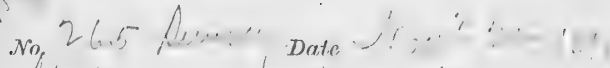

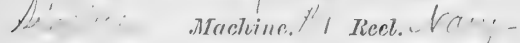
Tiuns
Cor. +29
Depth

Shot ointerest 60 tb\%

Botiom fring hic

Botlom temperature -

No. of thermometer

Cor. -

Corrected temperature

Air 76 Surface 7.7 1 Drift -

Trawl or aredge. -

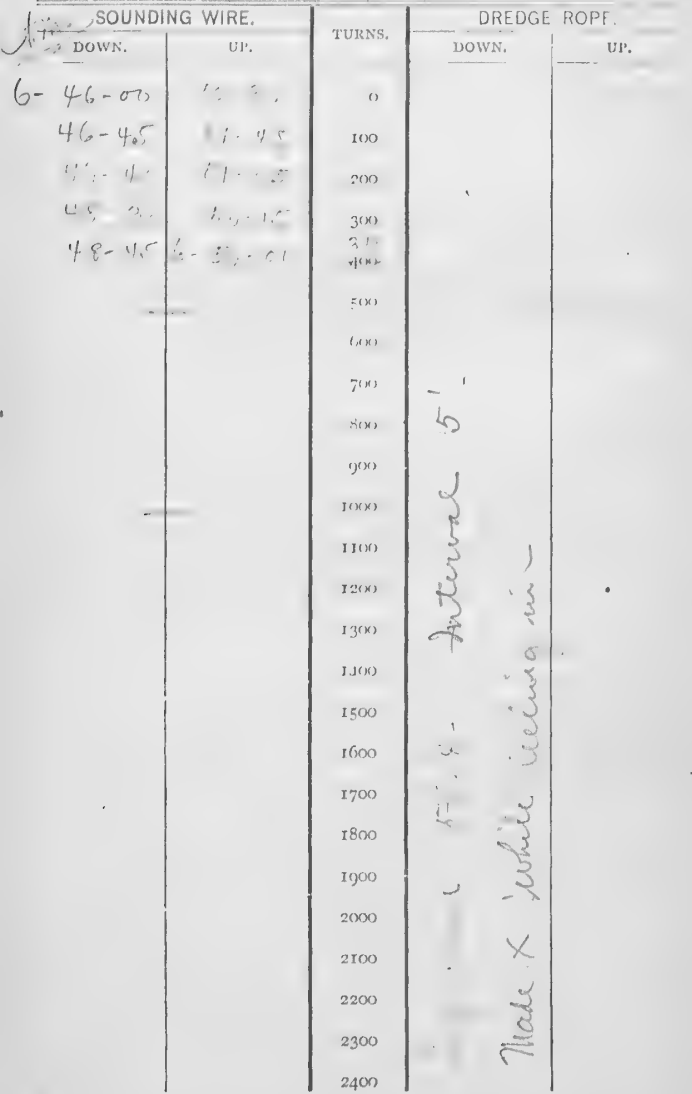




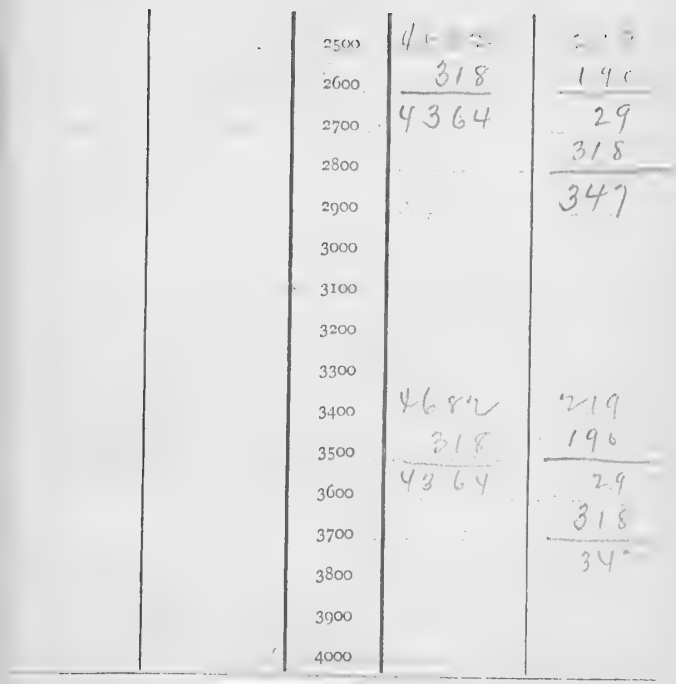

SERIAL TEMI'ERATURES.

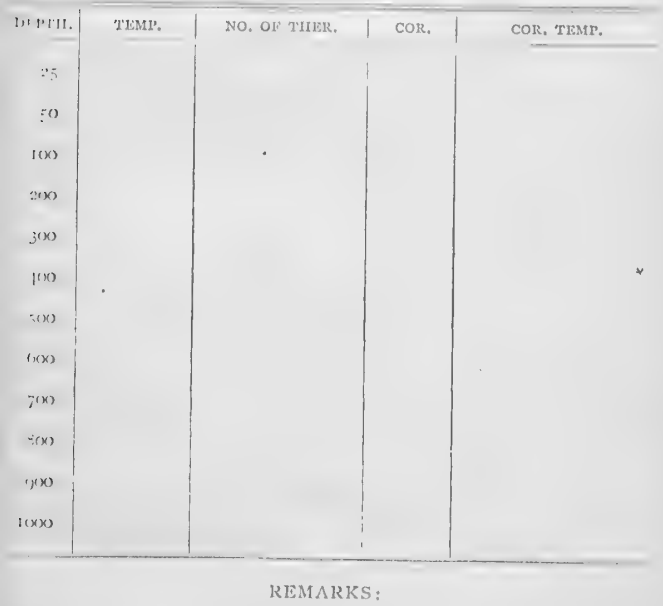


$2 q^{2} 2$

No.

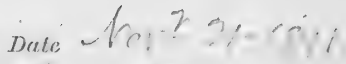

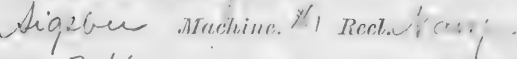

Turns $246 \mathrm{cor}+22$ ग0plin:-68

Shot 60 thr.

Bottom

$$
\text { yof } \mathrm{s}^{\mathrm{s}}
$$

Bottom temperature 45.0

No. of thermometer $6666.5 \quad$ Cor. $=0,2$

Corrected temperature 44,8

Air .7. 76 Surface 9) Drift -

Trawt or dredge -

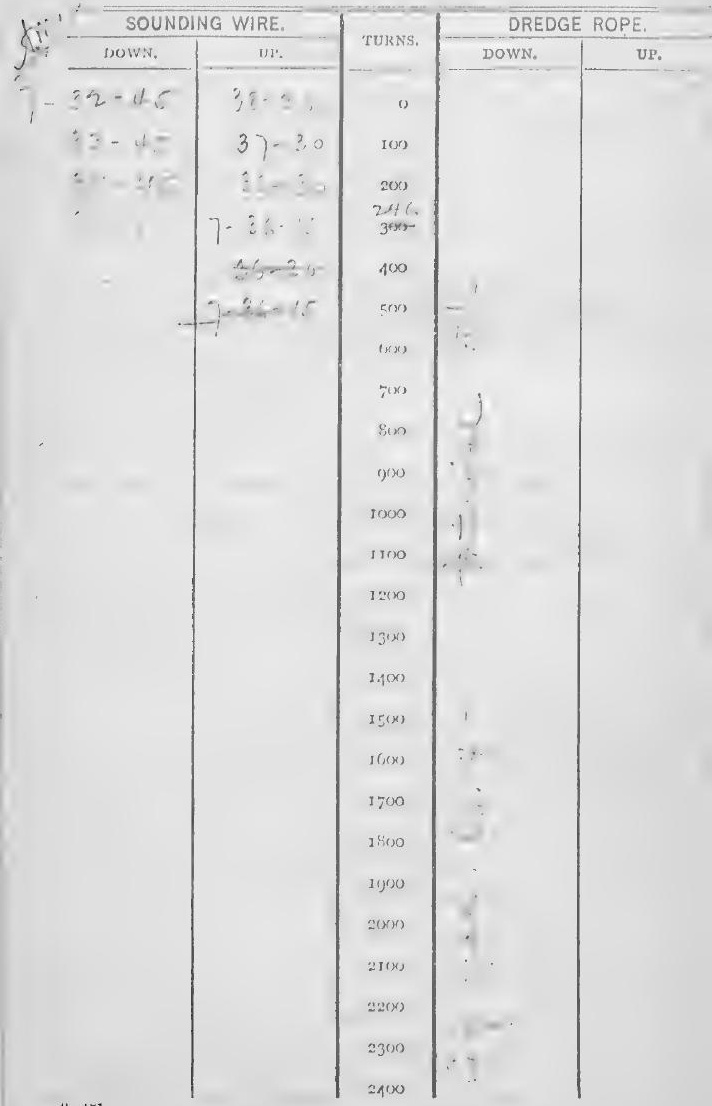




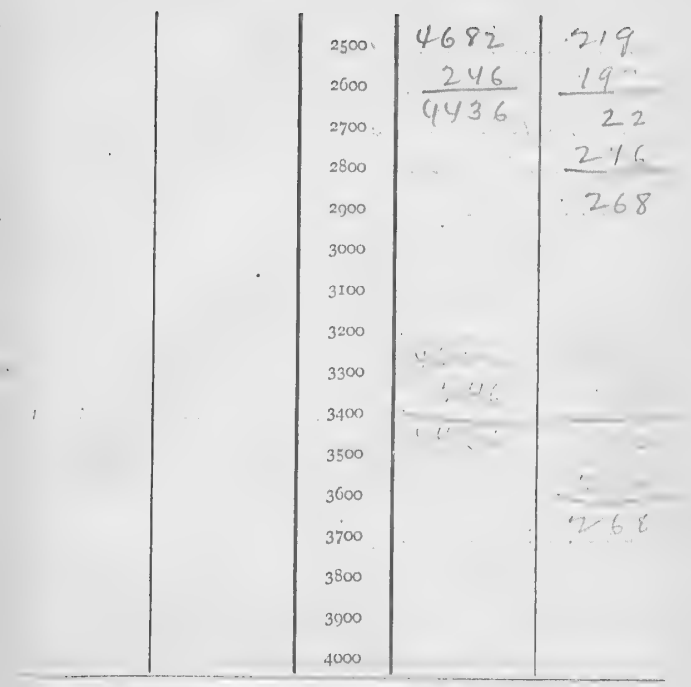

SERIAL TEMPLRATURES,

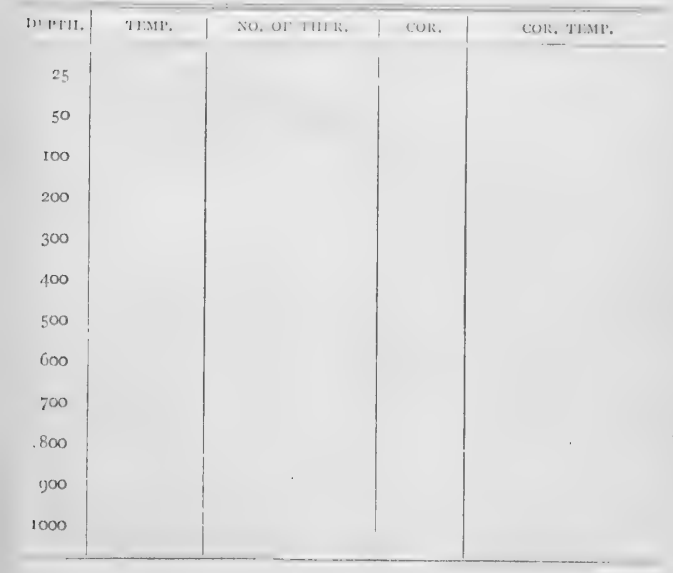

REMARKS : 
No.26\% Date

Machine" reei. P/ar.

Turns $\left.3 \sqrt{7} \mathrm{Cor}_{\mathrm{j}}+\right\}_{\mathrm{i}}$ Depth \&

Shot or tead (e-v

\section{Bottom}

Bottom temperature

No. of thormometer

Cor.

Corrected temperature

Air 7 Surface ' , Drift

Trawl or dredge

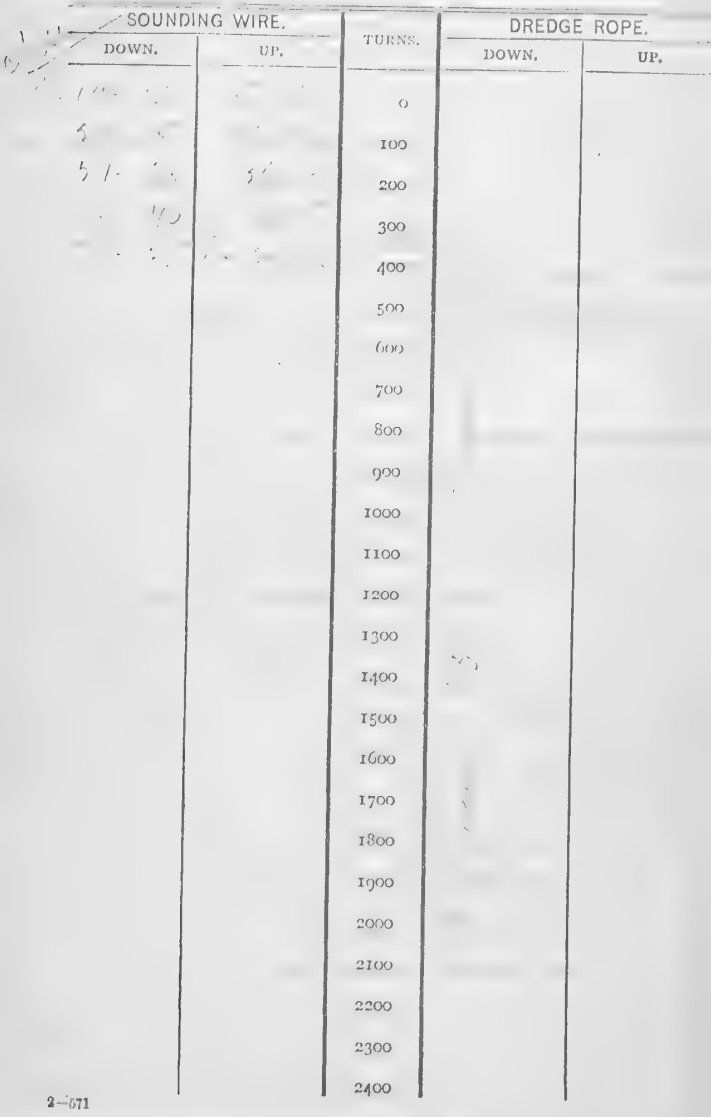


No.

$$
\text { Date } 1 / 1) ;
$$

'npect Machine. Reel.

CTums 275 Cor + Depth $50 \%$

57ver-br lead

$$
\text { i) } P \text { es }
$$

\section{Bottom}

\section{Bottom temperature}

No. of thormometer

Cor.

Corrccted temperature

Air. ? 7 Surface

$$
78 \quad \text { Drift }
$$

Trawt or dredge

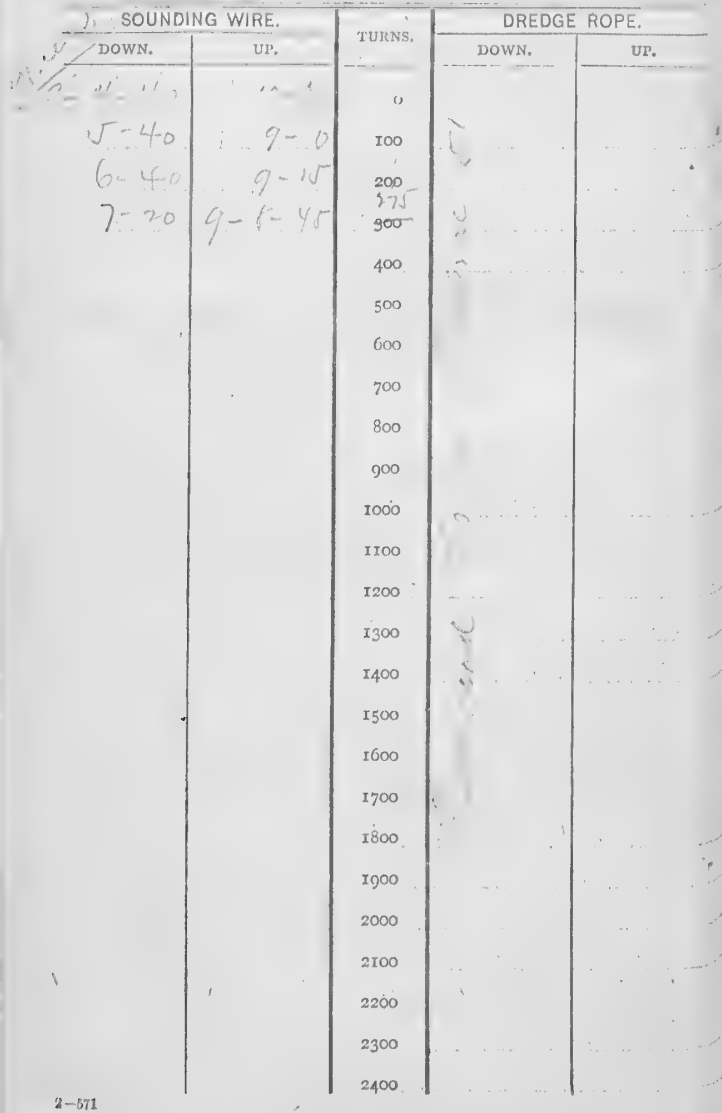




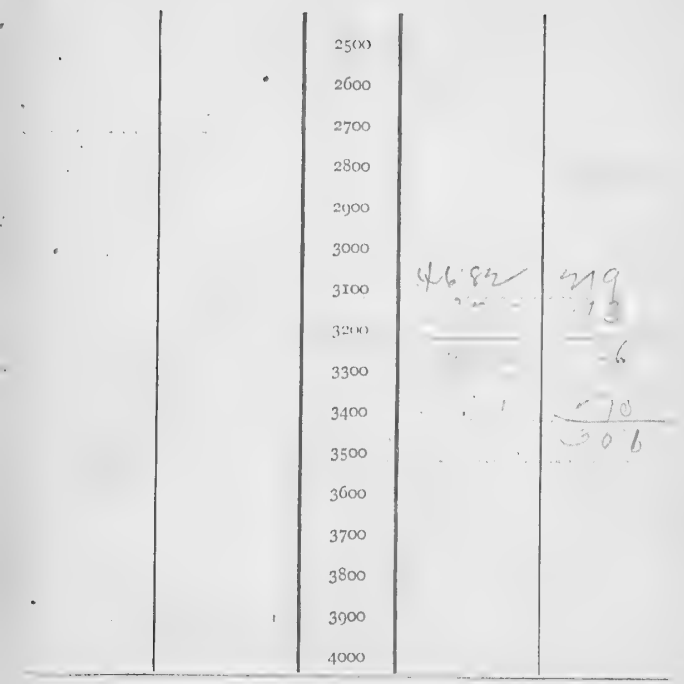

SLRIAL TEMPERATUIRES.

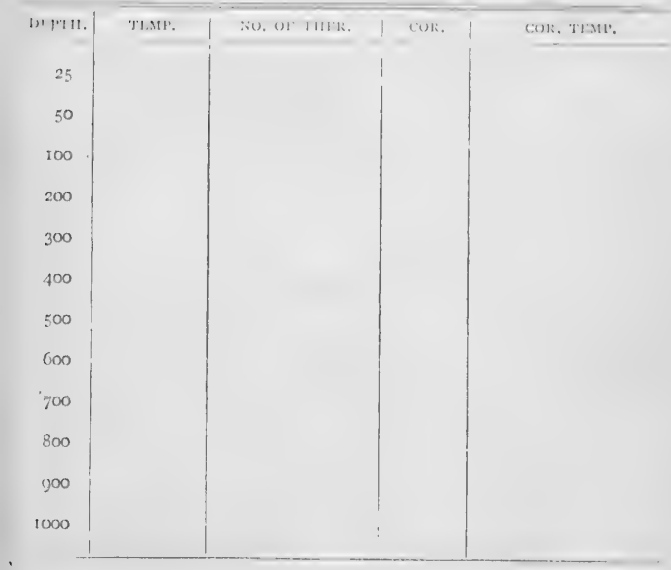

REMARKS : 
No. Date Wh W

Machine. "I Reel: Ui A.r.

Turns \& 6 Cor.,+ 9 Depth / 65

S7robur tead

Bottom

$$
Y_{1}^{1}, 11
$$

Bottom temperature

No. of thormometer.

Corrected temperature

Air<smiles>C1=CC2C[CH-]C12</smiles>

Sterface

Irawt or dredge

$\because$ SOUNDING WIRE.

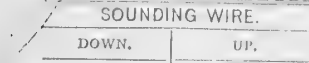
TURNS.

o

200

300

400

500

$\theta(x)$

700

800

900

I 100

1100

1200

1300

2400

I 500

1600

1700

1800

Iyoo

2000

2 ron

2200

2300

2400
Cor.

Drift

DREDGE ROPE

UP. 


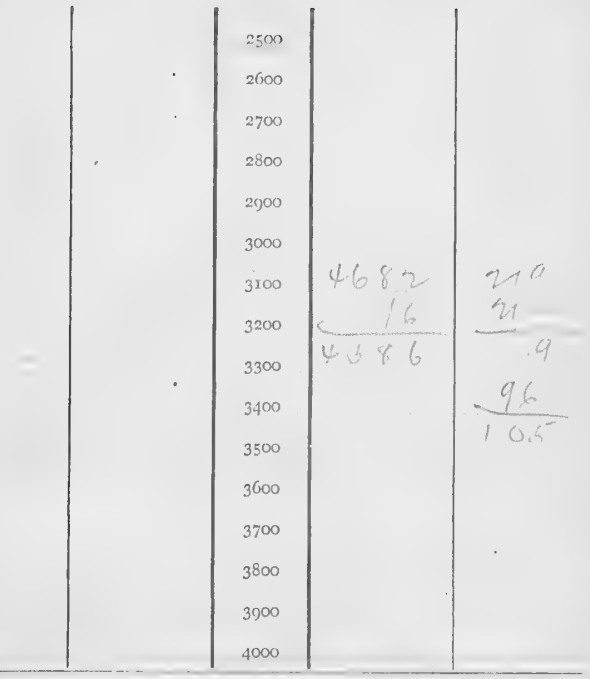

SERIAL TEMPERATURES.

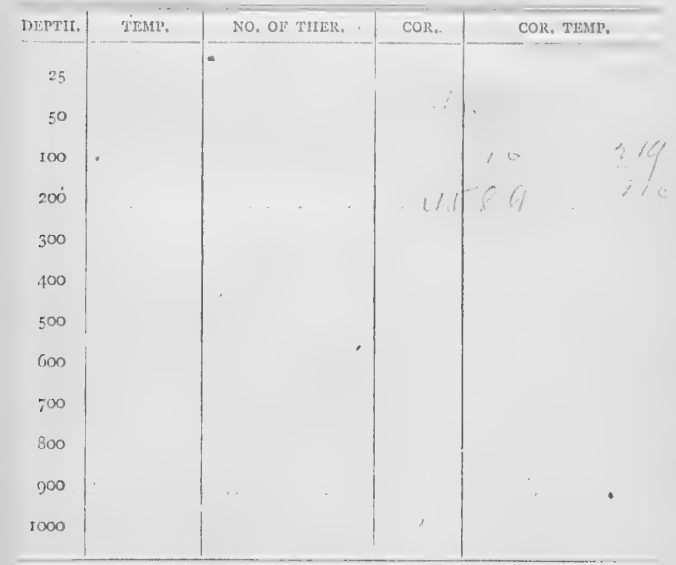

REMARKS : 
(1)

) $\left(\int^{\prime}\right) ?$

No.

Date

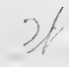

4. (C) Machine. / Reel. . Lit 'ं yे

Turns " i. Cor t $\therefore$ Depth

shotor lead . . 7f cicos.

\section{Botitom}

: Bottom temperature

No. of thermometer

6.65 Cor. $-0,2$

Corrected temperature

$43, \%$

Air .78 Surface 78 Drift

Thawl or dredge

$(1,1)$ SOUNDING WIRE.

DOWN, UP.
UP.

$\therefore c \div$.

$42-30$

(j) $\cdots 0$

o $2-0$

.

To

DREDGE ROPE.

DOWN.

UP. 


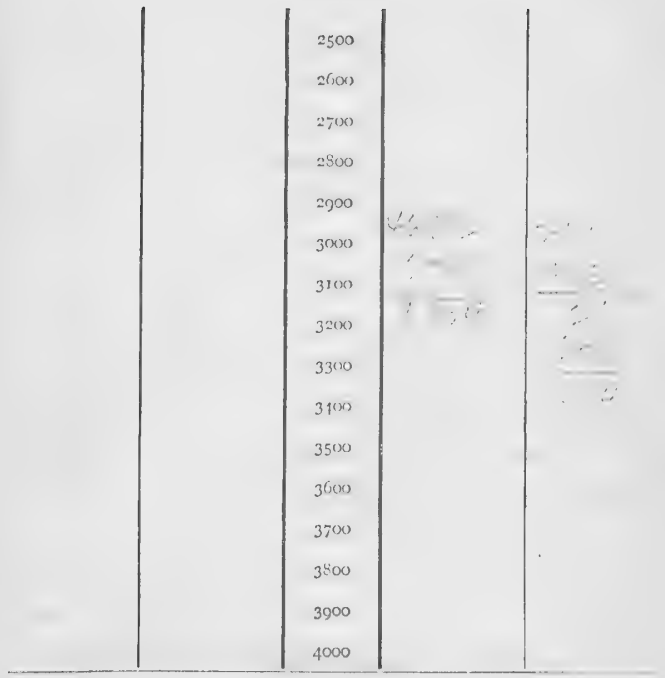

SERIAI, TEMPERATURES.

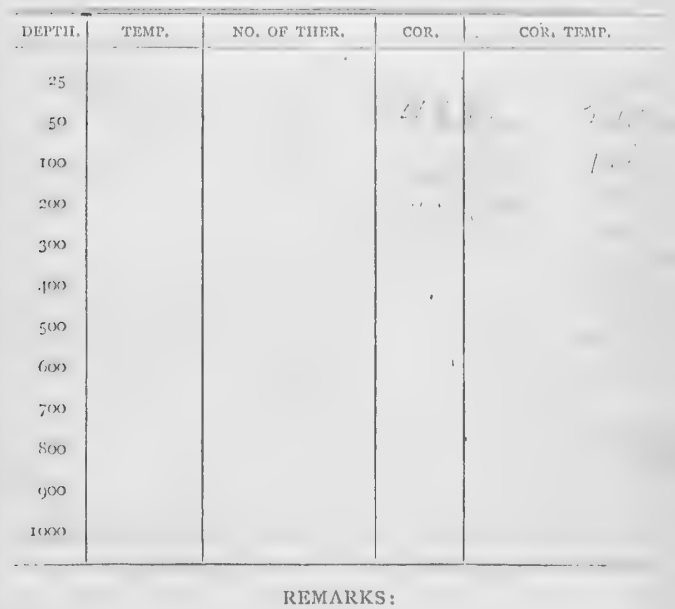


t:07.

No.

Date

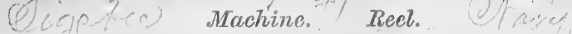

Turns Hol Cor + \& j Depth h?

shotion lead

follom.

Bottom temperature

No. of thermometer?

Cor.

Corrected temperature

Air 78 surface...78 Drift -

Trawl or dredge

SOUNDING WIRE. DOWN.

IVI.

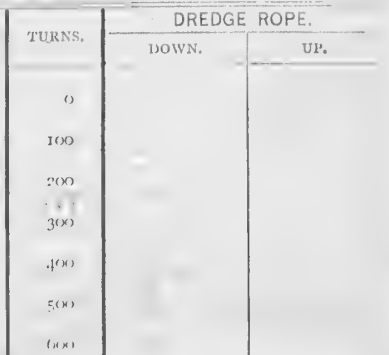




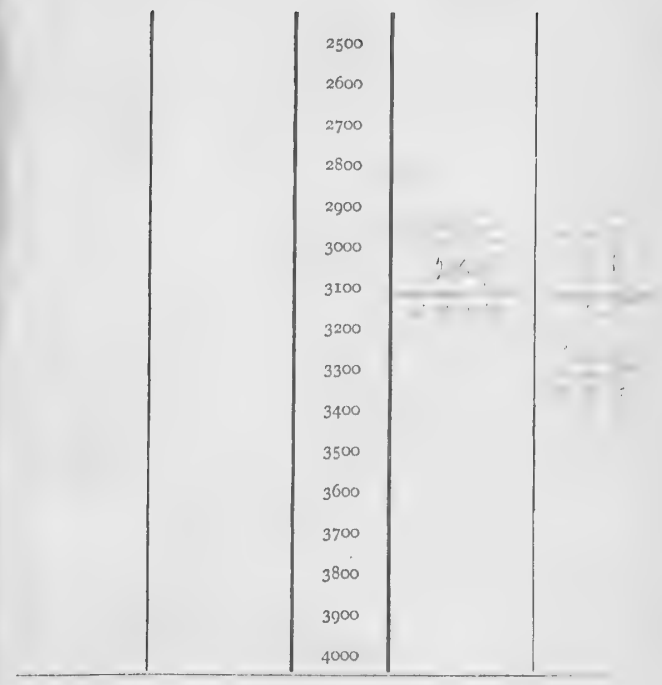

SERIAL TEMPERATURES.

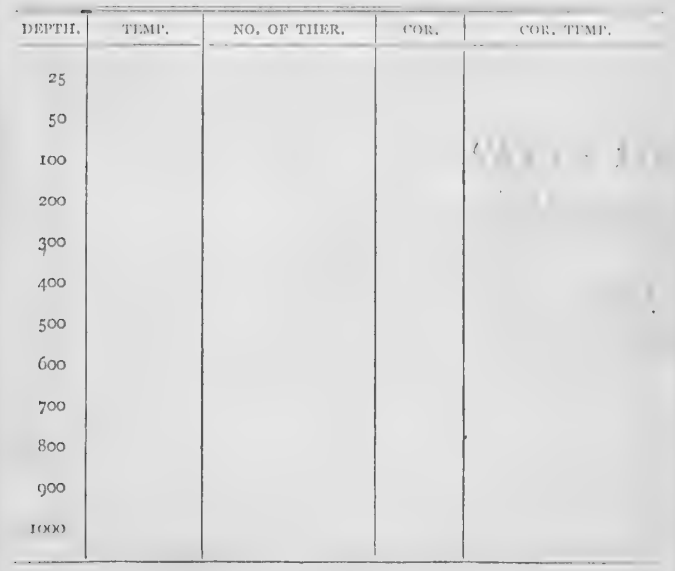

REMARKS : 
tor.

No.

Date WYY? 2-1

Machine. Al/ Reet.

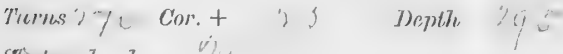

STitobor lead iें

Bottom hus whts

Bottom temperature

No. of thermometer

Cor. .....

Corrected temperature

Air 78 . Surface .. Drift

Trawt or dredge

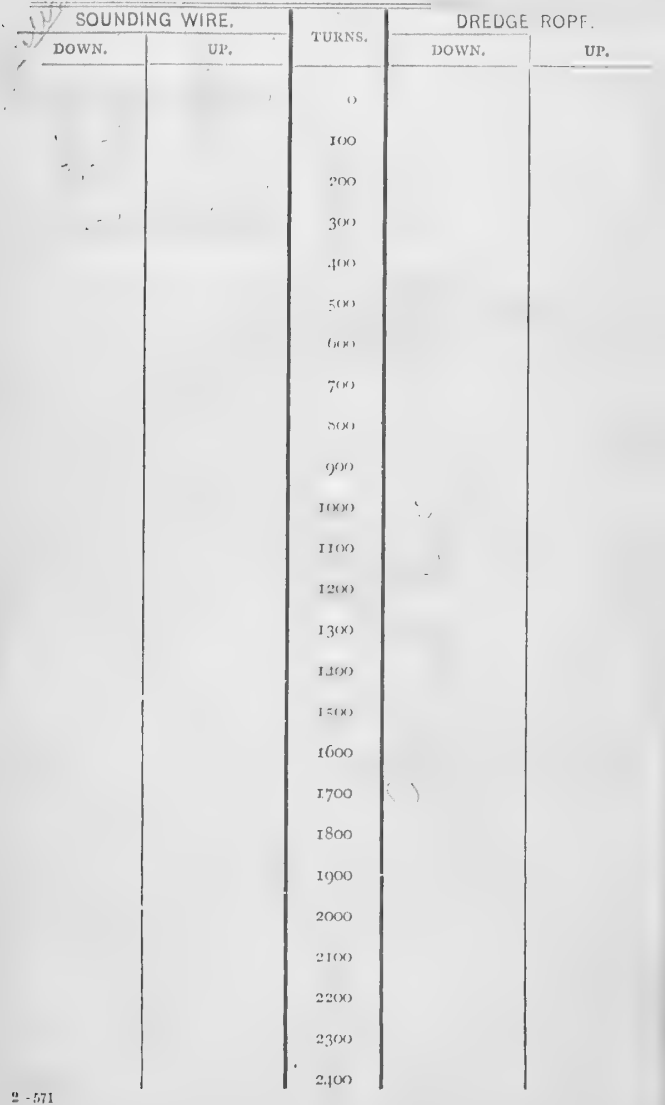




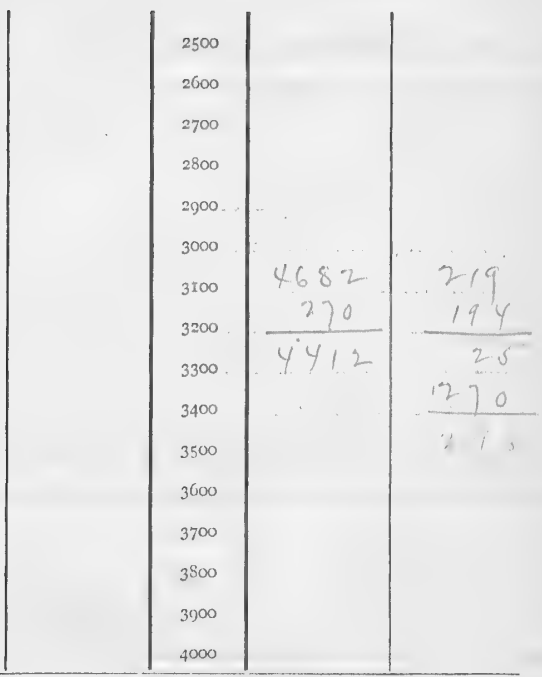

SERIAI. TEMPLRATURES.

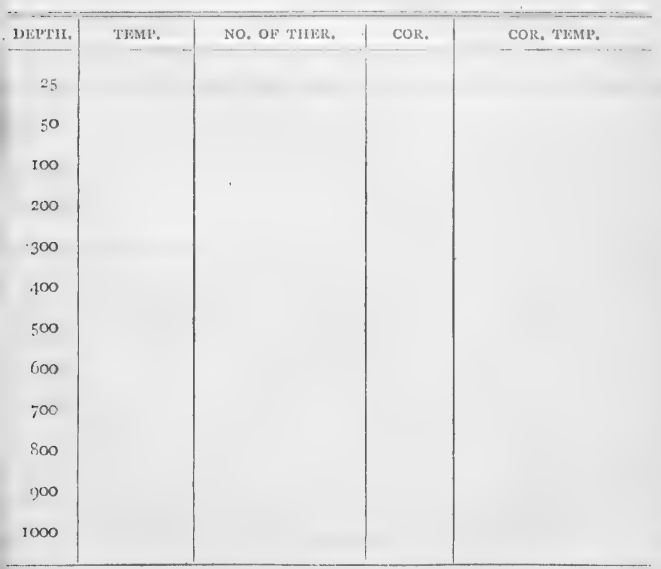

REMARKS : 
$\therefore \quad$ $\left.+; l_{1}+1\right), \ldots$

, No<smiles>CC=CC=C(C)OCC</smiles>
dlace Machine. Reet.

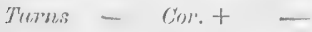

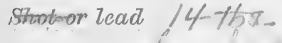
$7+2 \div / 8$

Bottom rin. OS, Co

Bottom temperature

No. of thermometer

Cor. -

$\therefore$ Corrocted temperature

Air 77 . Surface 78 . Drift

Trawt or dredge

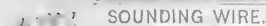

$1, \cdots$, SOUNDING WIRE.

J) WN.

- UI.

$12-10-00 ;=$

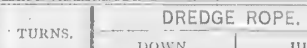

Ior)

2on

300

400

Eron

fit)

7(ir)

$S(x)$

joo

I000

I I0O

1200

1500

I. 100

1500

I 600

1700

1800

$I(y) 0$

2000

2100

2200

2300

2400

Jepth 10 fin. 


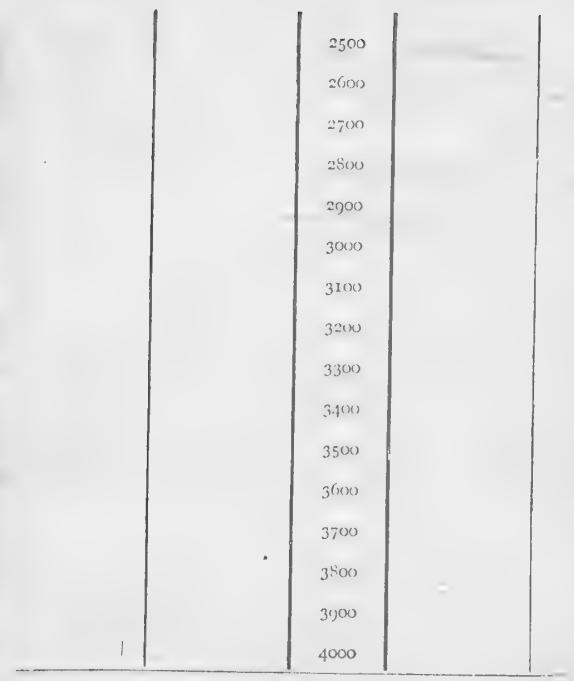

SERIAL TEMPERATURES.

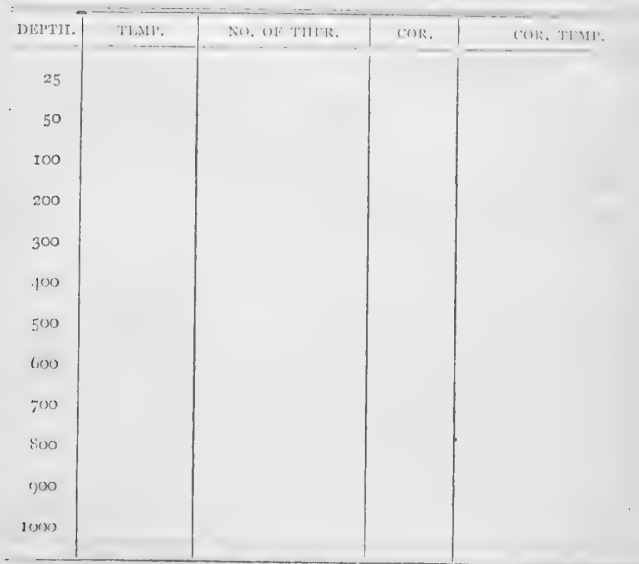

REMARKS : 
So.

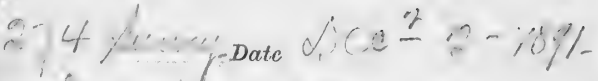

Machine. Reet.
Turns
Cor. +
Depth

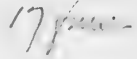

shatum zeart $14-16 \mathrm{~s}$,

Bottom Co.

Bottom temperature

No of thermometer.

$\therefore$ Cor.

Corrected temperature

Air 77 .

Air .... 7.7

Surface

77 Drift

Traul or dredge

Pl, SOUNDING WIRE.

JDOWN. UN.

$1: 4.5 \cdot 6$

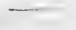

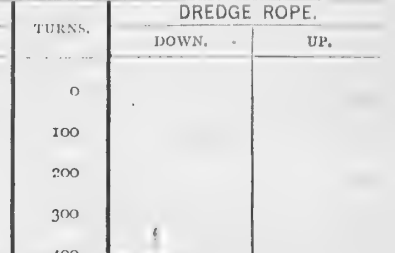

50

G(x)

$7(x)$

$8(x)$

900

I $0 \times 0$

IIOO

T200

1300

I. 100

$15(10)$

Ifon

1700

J 800

$J(y)$

2000

IIOn

2200

2300

$2.10 n$ 
tor.

so. 275 , Lerwary Date

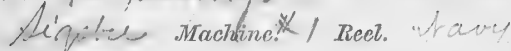

Turns 20 . Cor. +

strot oulead 38 tbs'.

bisllom.

Co.t 7 ritid,

Bottom temperature

No of thermometer

Cor.

Corrected temperature
Air
Surface
$\%$ Drift

Trawt or dredse

SOUNDING WIRE.

pows.

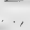

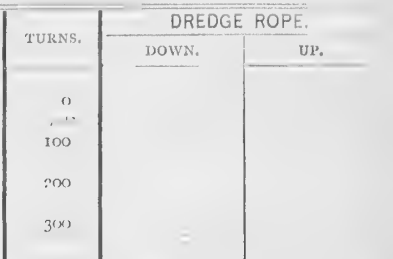




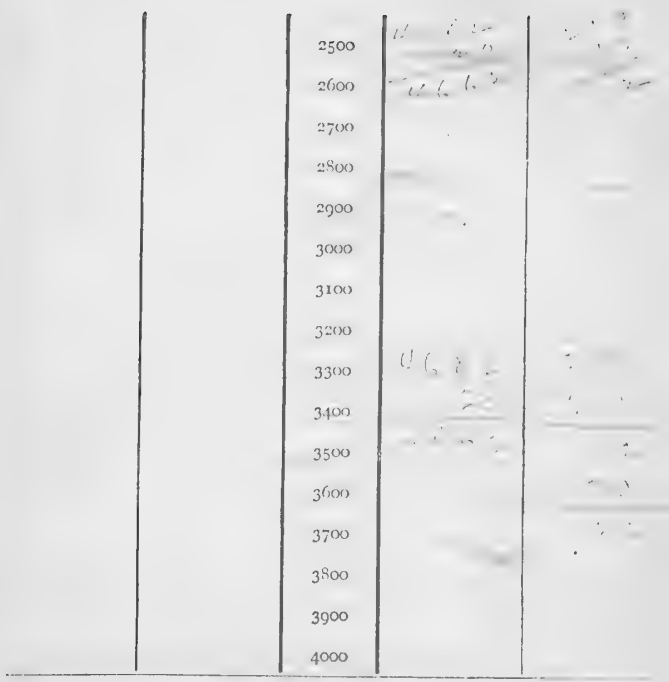

SERIAL, TEMPERATURES.

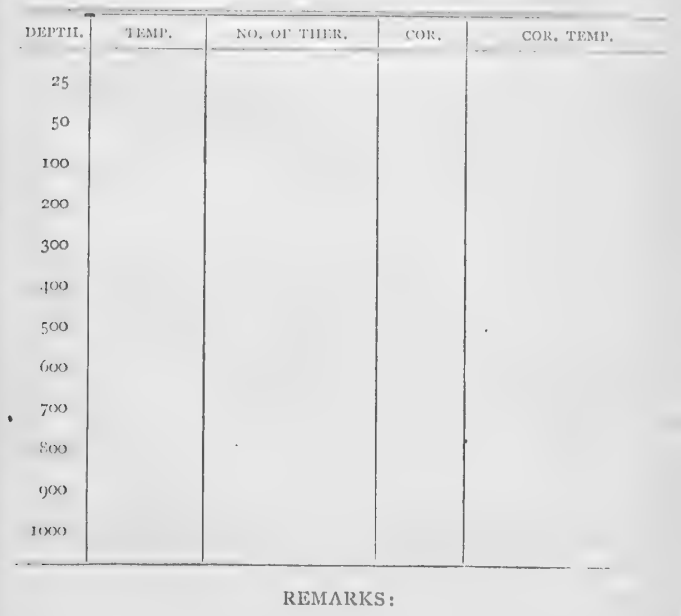


ron.

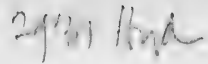

. vo.276 1-cerecé, Date

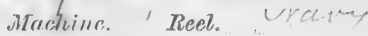

Túrms 4 Cor. 4 Depth 47 fmo -

statior lead 38 trom.

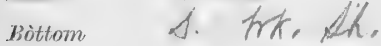

Bottom temperature

No. of thermometor

Cor.

Corrected temperature

Air

Surface

Drift

Trawl or dredge

SOUNDING WIRE.

DOWN.

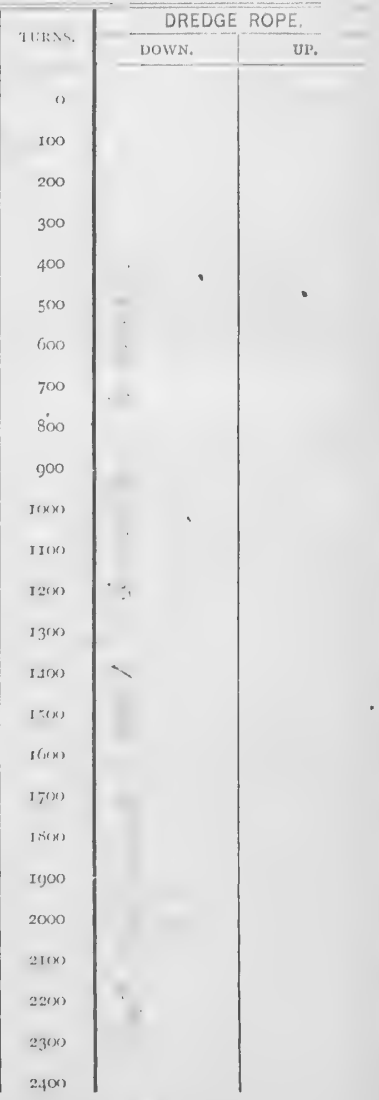




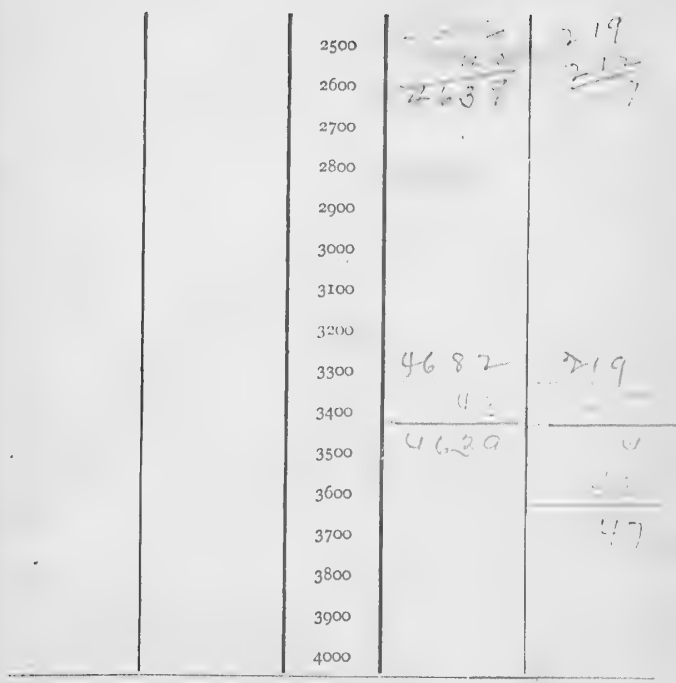

SERIAL TEMPERATURES.

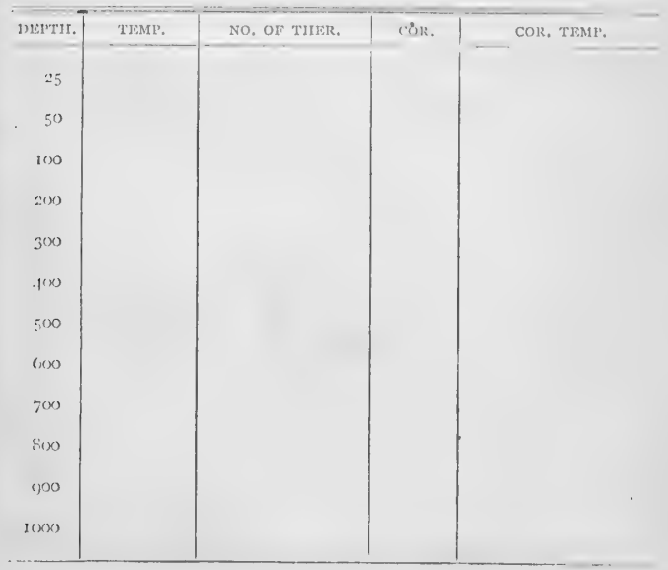

REMARKS : 
No. 27 U1, :

$\therefore$ Machine. Mech. Mravy

Turnis

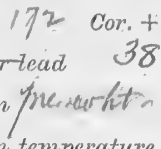

sthot in-lead 38

Depth

189

Bottom frerdurfitin

Bottom temperature

No. of thermometer

Cor.

Corrected temperature Ai,

Surface

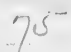

Drift

Trawt or dredge

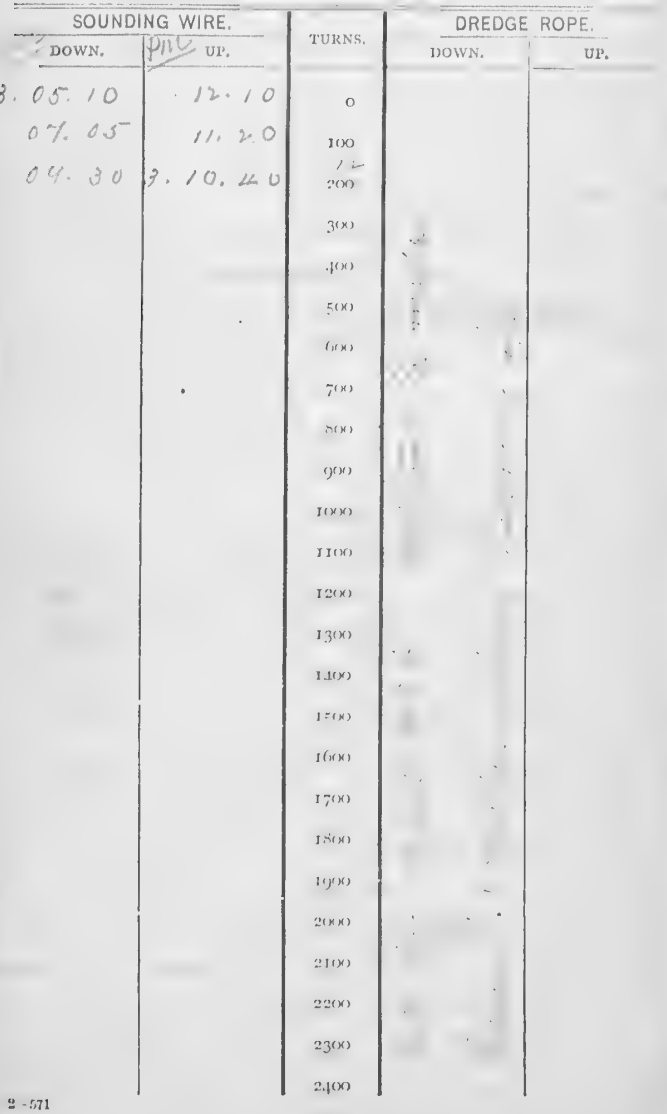



2933

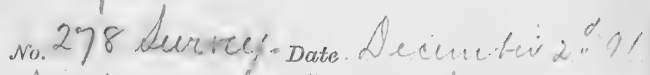
suqua 4 Machine." Reer. Arave.

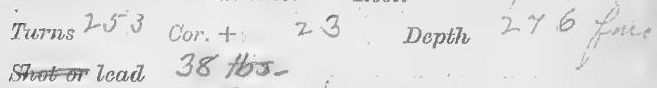

Bottom

Bottom temperature

No. of thermometer

Cor.

Corrected temperature

Air. 76

Surface

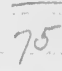

Drift

Trawl or dredge

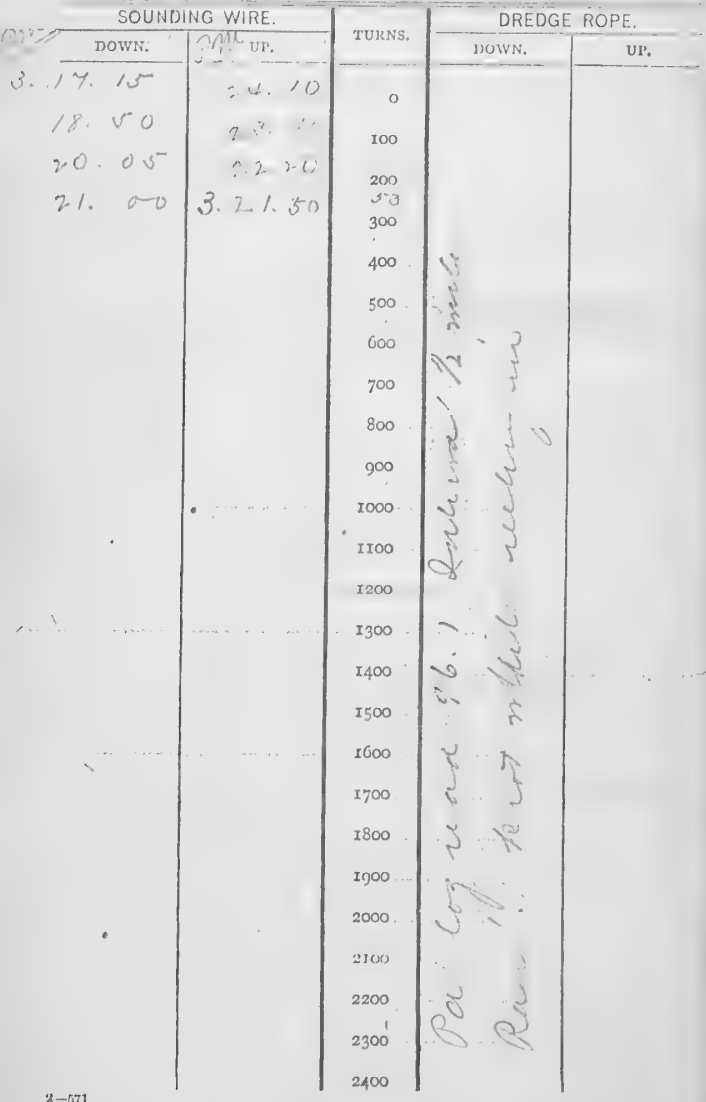




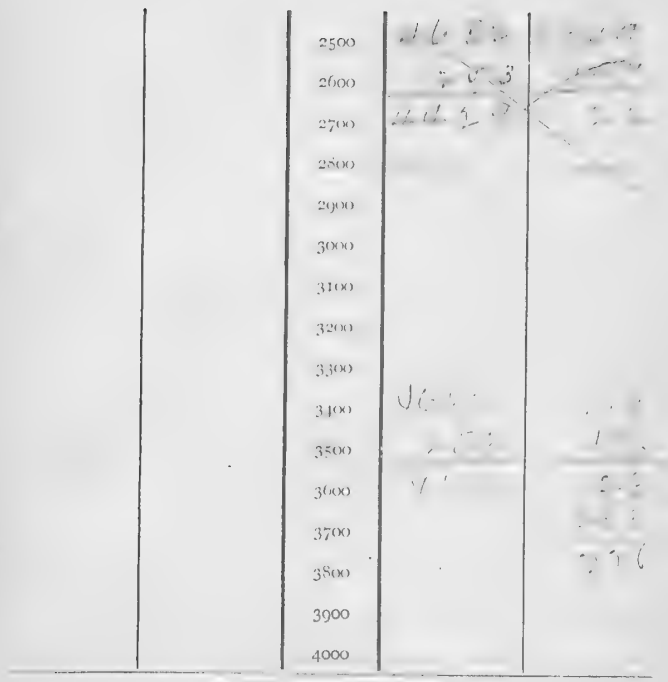

SERIAL TEMPERATURES.

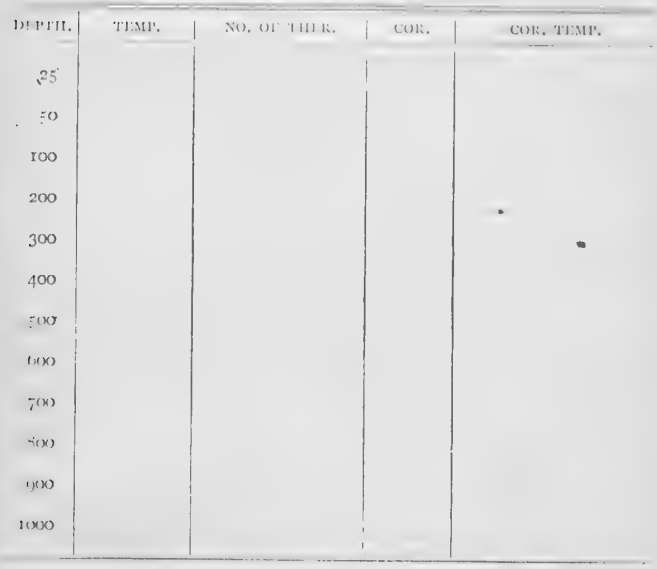

REMARKS : 
so.279. devray_Dute

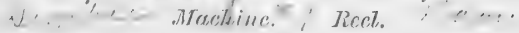

Turns 260 Cor + is Depth $285^{5}$

Shominglead 38 thss,

Bottomfucuitht. I.

Bottom temperature $44 \mathrm{C}$

No. of thermometer $62358 \quad$ Cor. -0.2

Corrected temperature $44.4=$

Air 76 ... Surface 75 ... Drift

Trawt or dredge

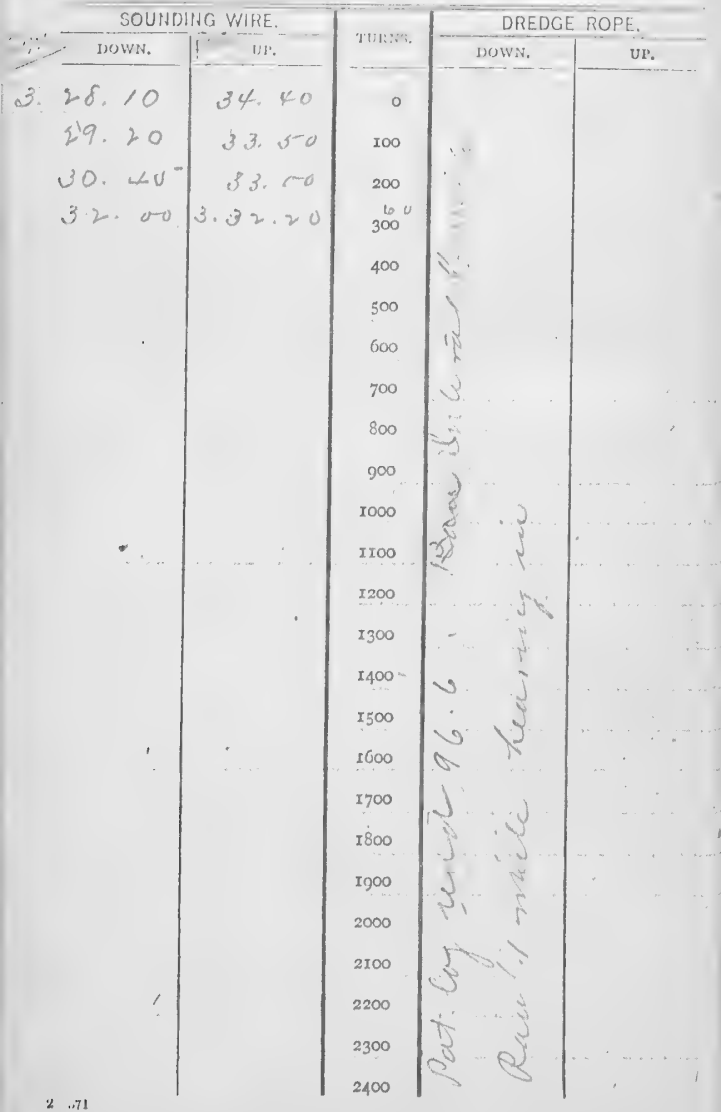




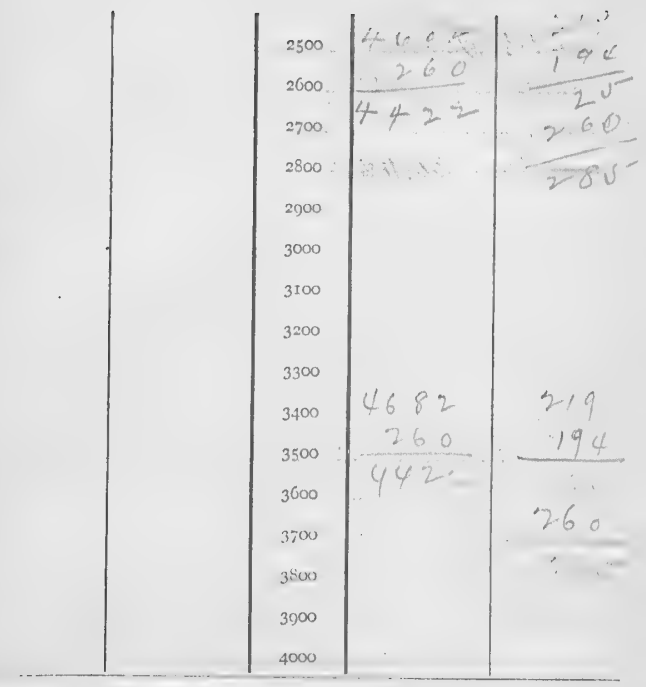

SERIAL TEMPERATURES:

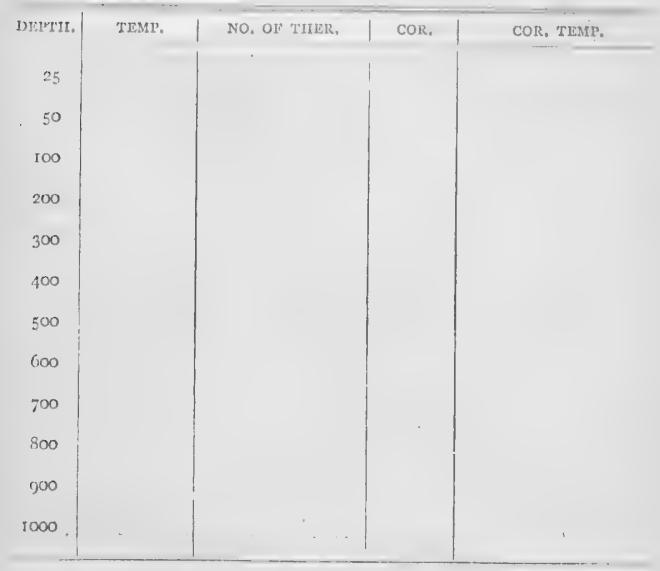

REMARKS : 
ris.

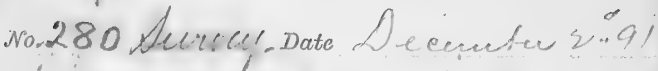
Libibec Machine: 1 Reel. Mat

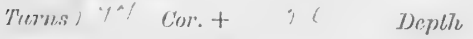

that lead 38 tos.

Bottom - C. C.

\section{Bottom temperature}

No. of thormometer

Cor.

Corrected tomperature

Air 76 Surface

Drift

\section{Irawt or dredge}

SOUNDIHG WIRE.

DOWN, UP.

3

$3 \times 20$

40.35

42.05

$2.05,3.11,0$

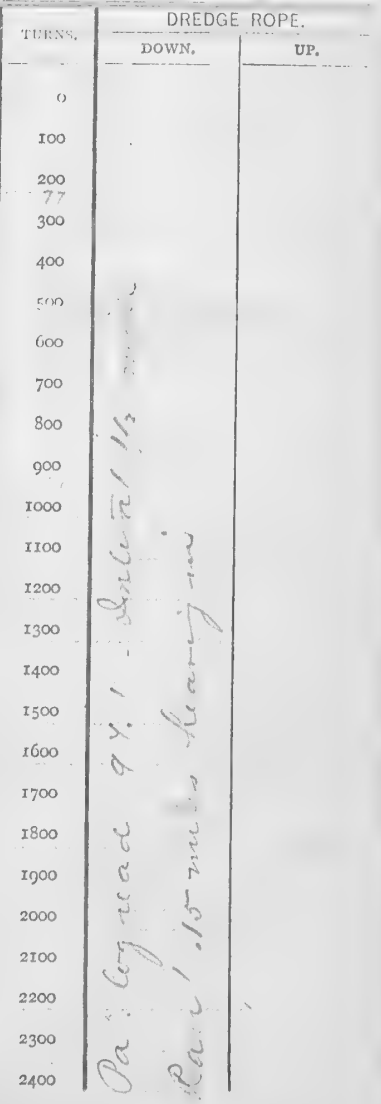




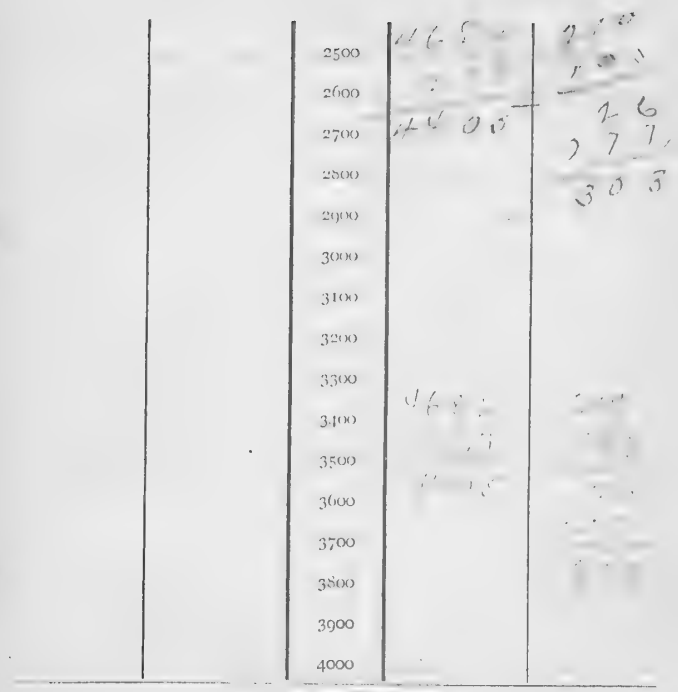

SERIAL TEMPERATURES,

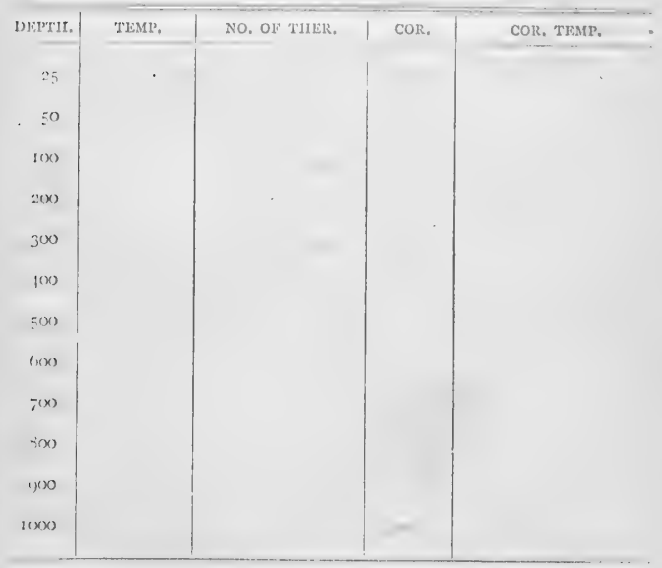

REMARKS : 


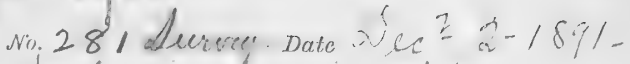
1) bee Machine " Rectstain -
Turus? ? If Cor. +21
Depele :55!

Shot 38 the

Bottom pre jón. S. Lavare.

\section{Bottom temperature}

No. of thermometer

Cor. -

Corrected temperature

Air 7.6

Szerface

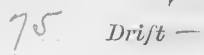

Trawt or dredge

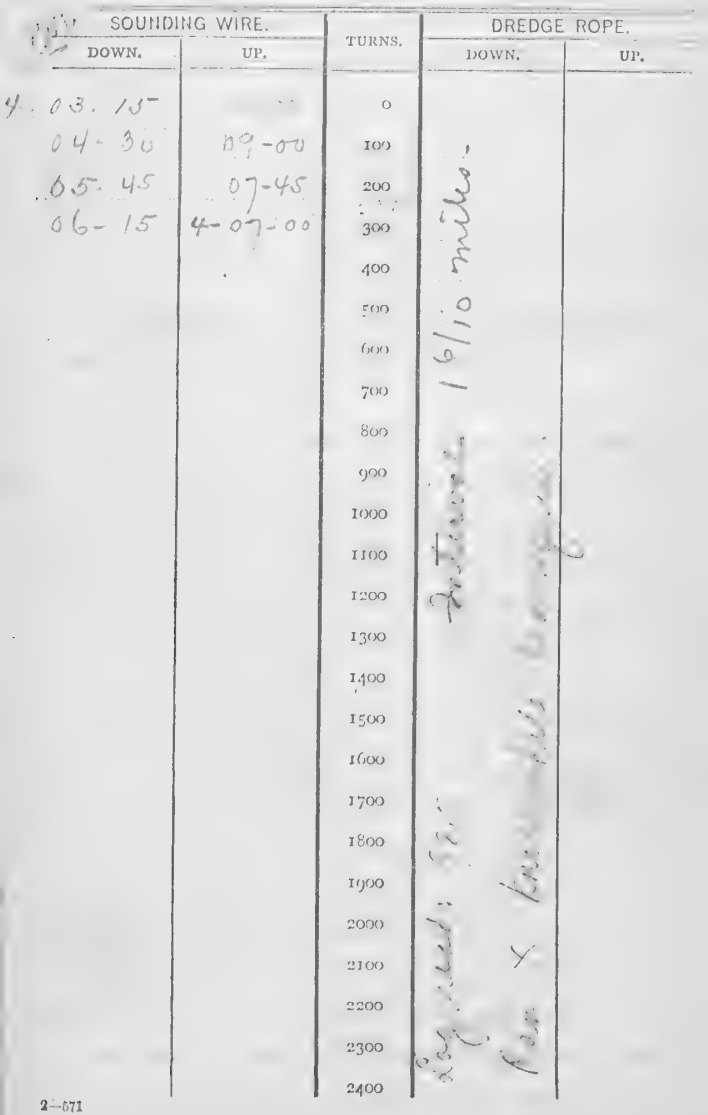




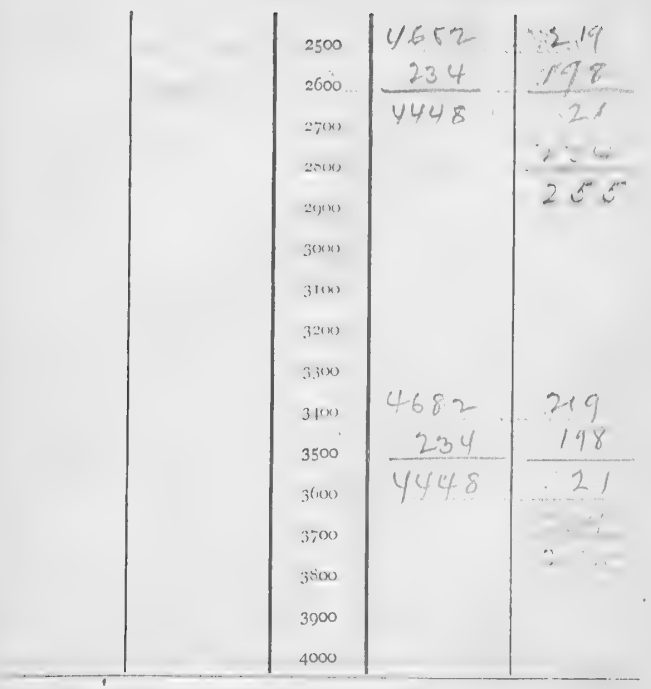

SERIAL TEMPERATURES.

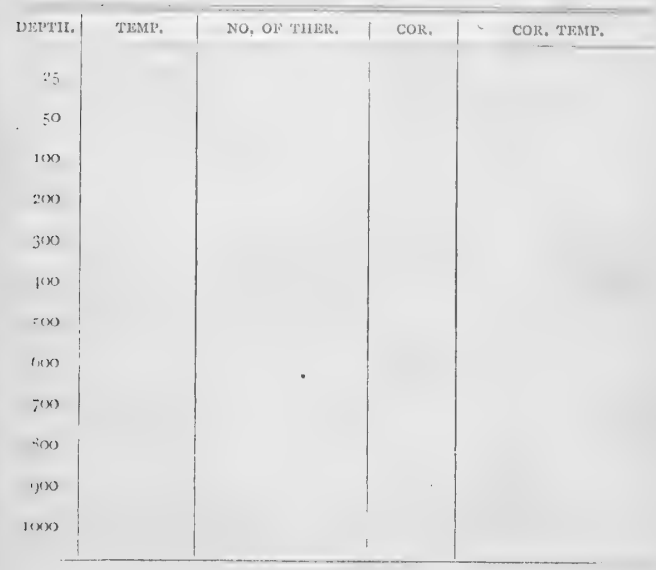

REMARKS : 


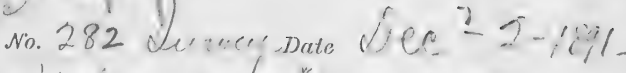
Ligevee machtime Thin 43
Cor. +4
Deptle 47 f:e.

steand $35 \%$ r.

Bottom Co. wh. S.

Bottom temperature

No. of thormometer

Cor.

Corrocted tomperature

Air 76 Surface 75 Drift

Trawt or dredge

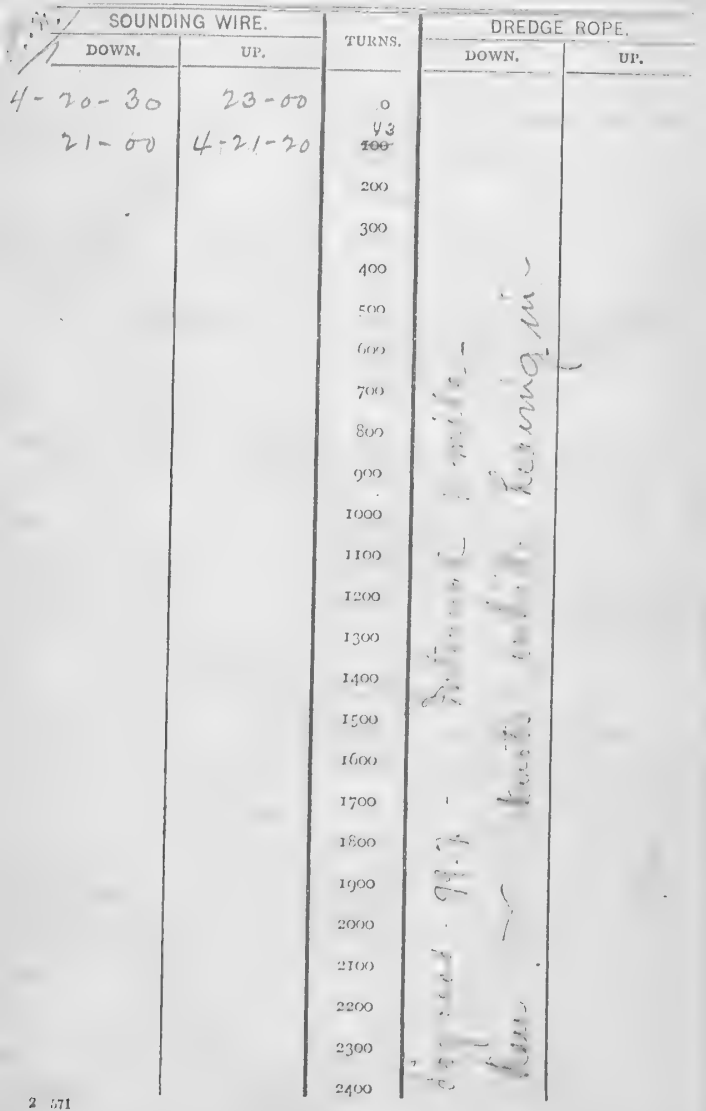




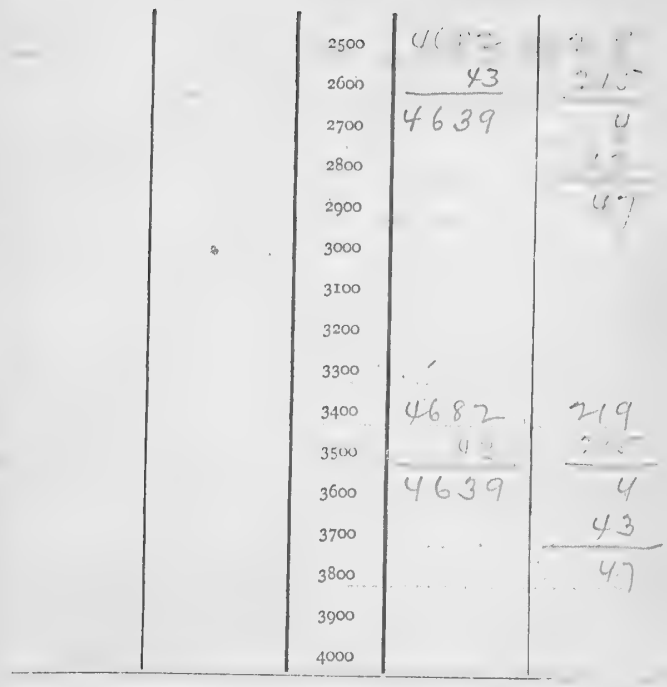

SERIAL TEMPERATURES,

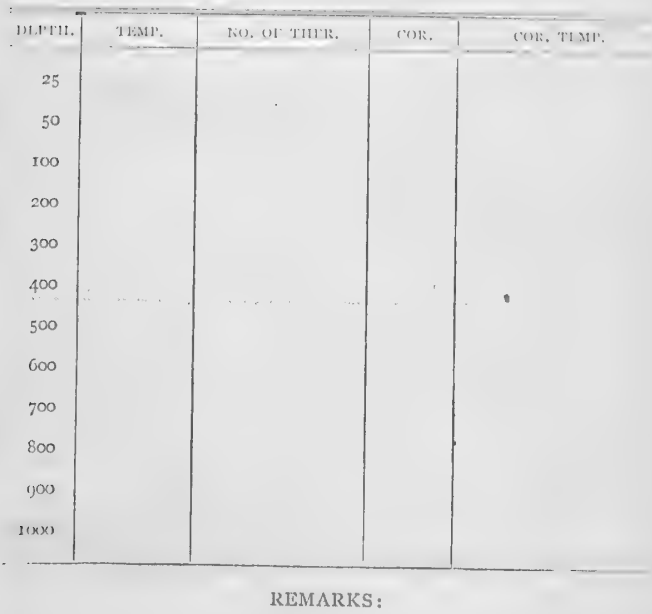


nor.

.No. 283

d)ect2-1891-

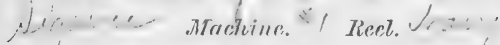

Turms 130 Cor +12

stroteren lead 38 tos.

Depth $142, \cdots 0$.

Bottom fue, whe d.

Bottom temperature.

64,2

No. of thermometer 62358

Corrected temperature 64,2

Air...76 Surface 75 Drift -

\section{Trawt or dredge -}

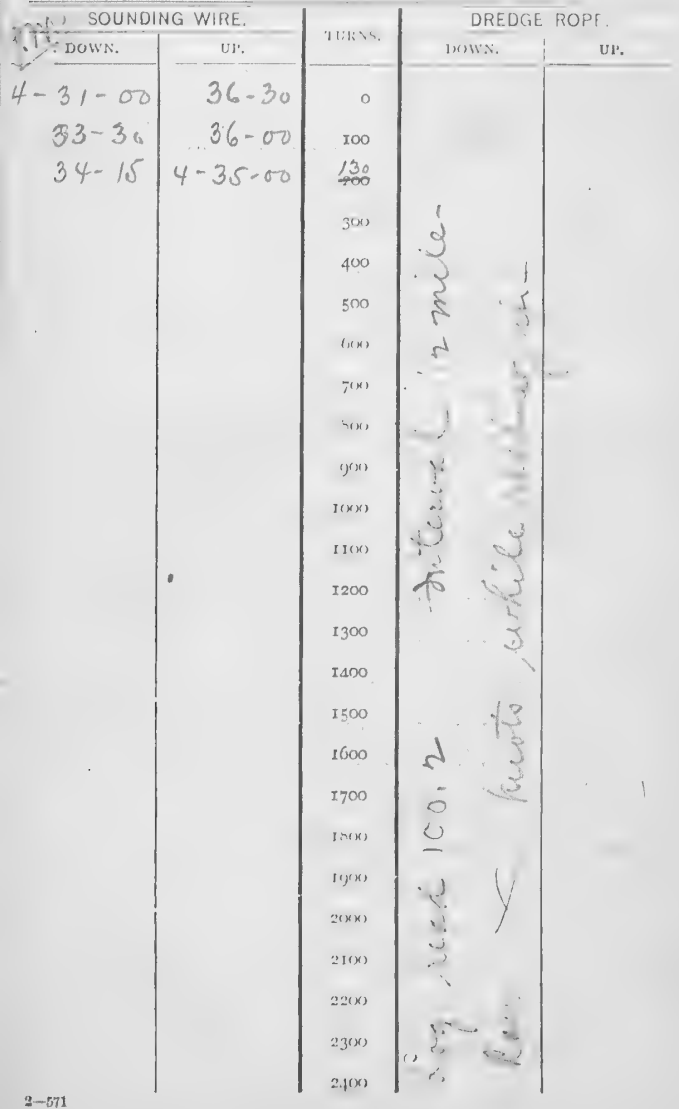




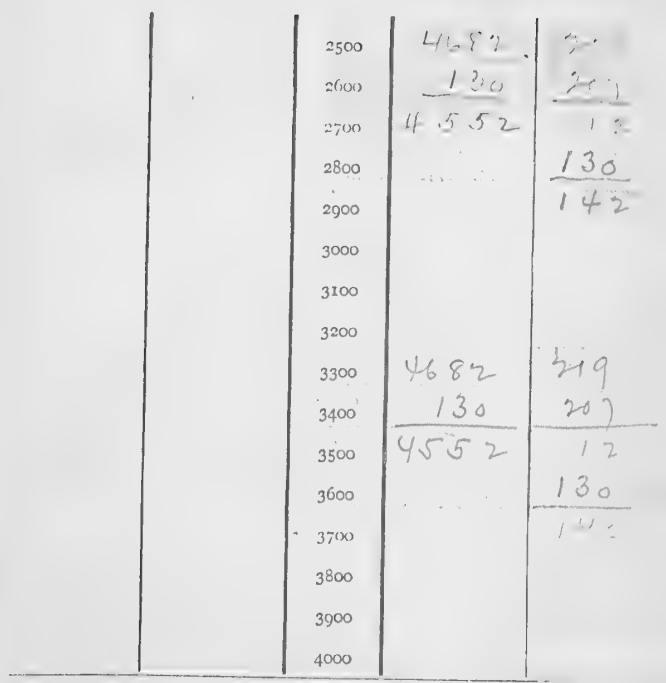

SERIAL TEMPERATURES.

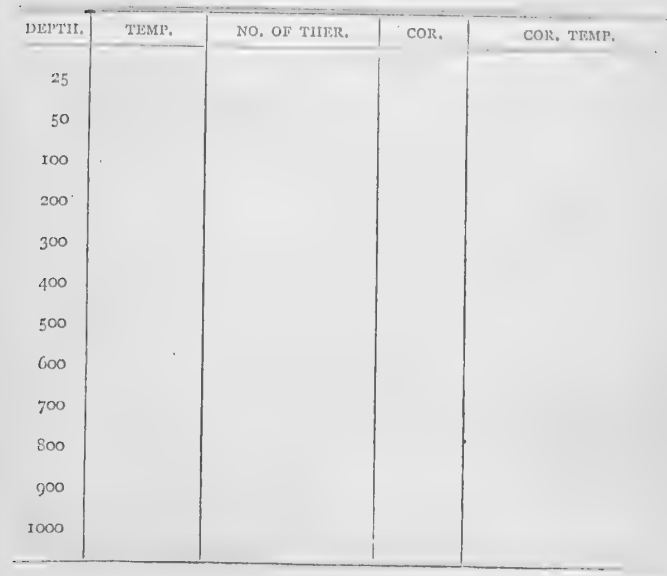

REMARKS : 
V.रि?

$\log 2$

No. 284 Date 1.
Turns 19. Cor. +2
Depth 21 frno-

sheotenr Tead 38 tbs

Bottom .. Whit s curac Sh.

Botlom temperature

No. of thermometer

Cor.

Corrected temperature
Air . . 76
Surface
Drift

Traut or dredse -

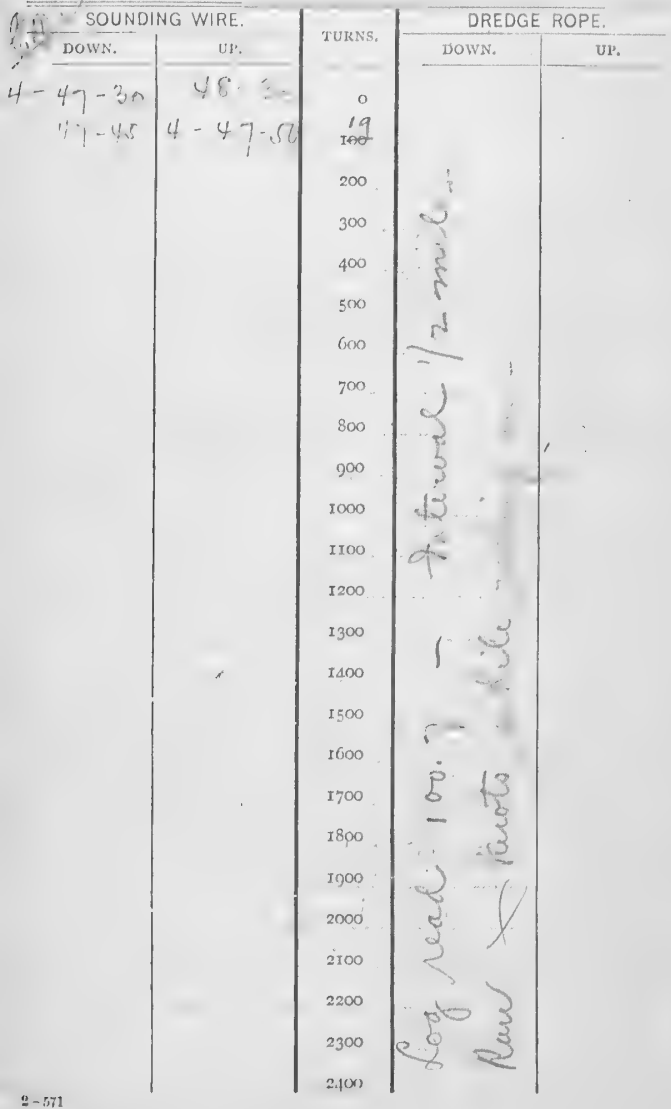




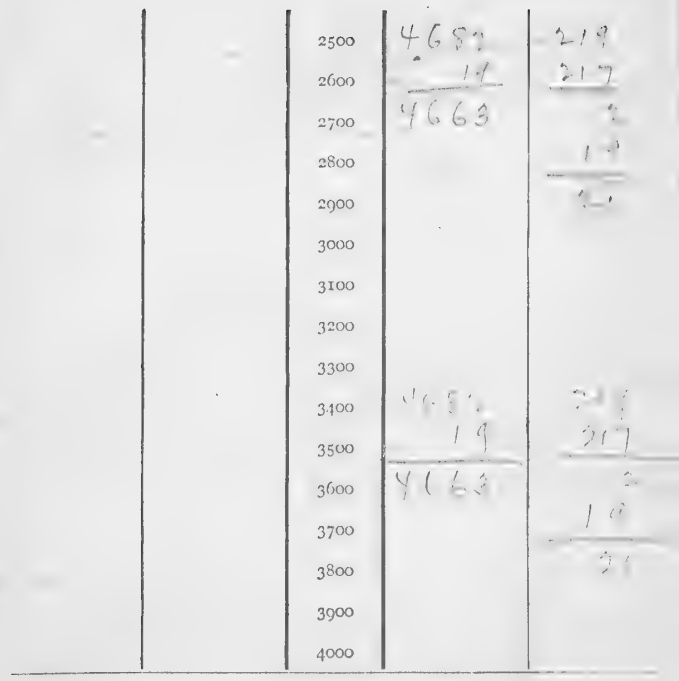

SERIAI, TLMPERATURES.

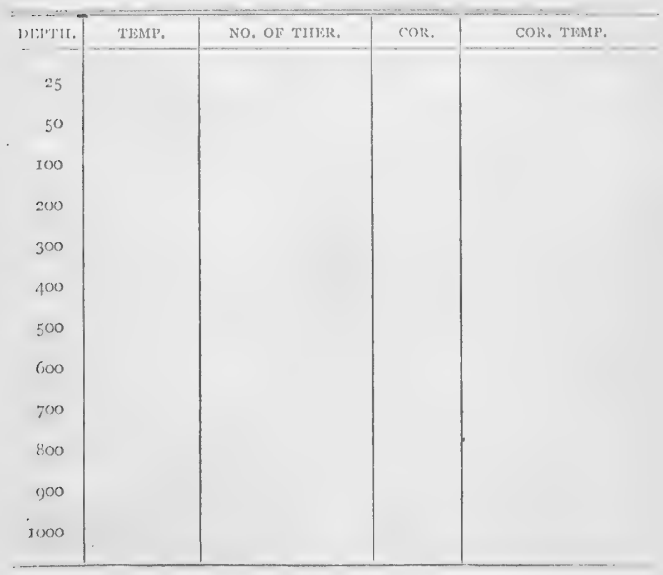

REMARKS : 


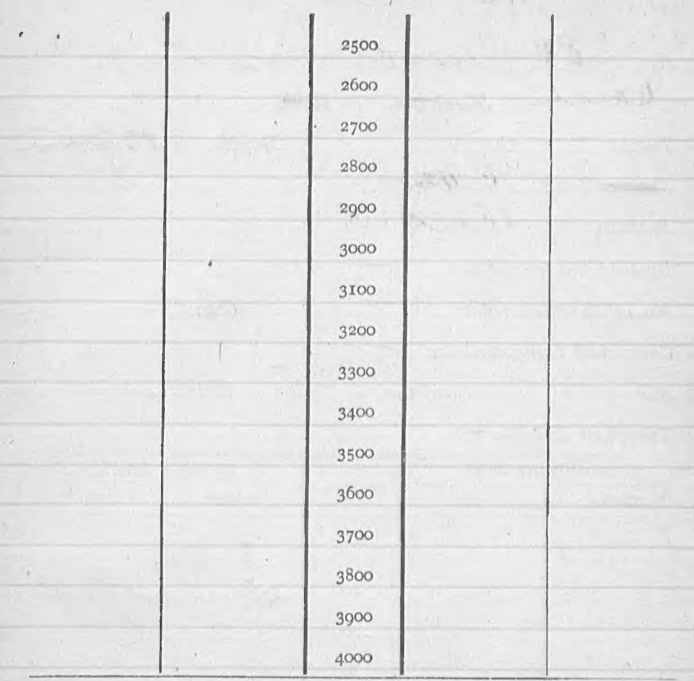

SERIAL TEMPERATURES.

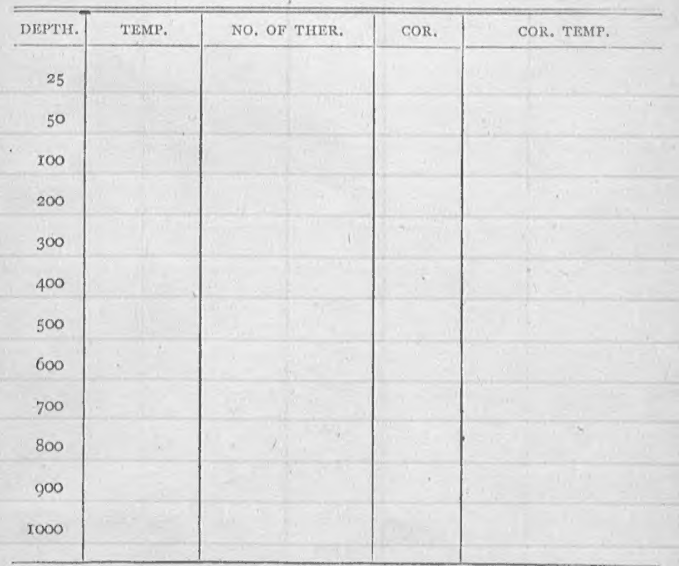

REMARKS : 
Hauk matome. Reet.
Turns
Cor. +
Depth
sead 14 to

Bottom

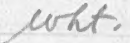

Bottom temperature

No. of thermometer

Cor.

Corrected temperature

Air 76 Surface 75 Drift

Traul or dredge

\begin{tabular}{|c|c|c|c|c|}
\hline$\ldots$ sout & IRE, & TURNS. & DREDGE & ROPE. \\
\hline DOWN. & UP. & 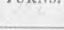 & DOWN. & UP. \\
\hline $5=0$ & & 。 & 8 & \\
\hline & & 100 & & \\
\hline & & 200 & & \\
\hline & & 300 & & zat \\
\hline & & 400 & s) & \\
\hline & & 500 & 2 & 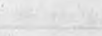 \\
\hline & & 600 & & 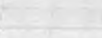 \\
\hline & & 700 & & \\
\hline & & 800 & & \\
\hline & & 900 & & \\
\hline & & 1000 & & tret \\
\hline & & $x 100$ & & $i_{1}$ \\
\hline 10 & & 1200 & $\nabla$ & lis \\
\hline t & & 1300 & 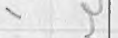 & $y^{2}$ \\
\hline & & 1400 & 6 & (2) \\
\hline & & 1500 & & . \\
\hline & & 1600 & & 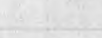 \\
\hline & & 1700 & & the \\
\hline & & 1800 & $\alpha$ & 20 \\
\hline & & 1900 & $J$ & . \\
\hline & & 2000 & 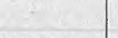 & 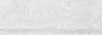 \\
\hline - & & 2100 & & \\
\hline & & 2200 & & \\
\hline & & 2300 & & \\
\hline & & $240 n$ & & \\
\hline
\end{tabular}




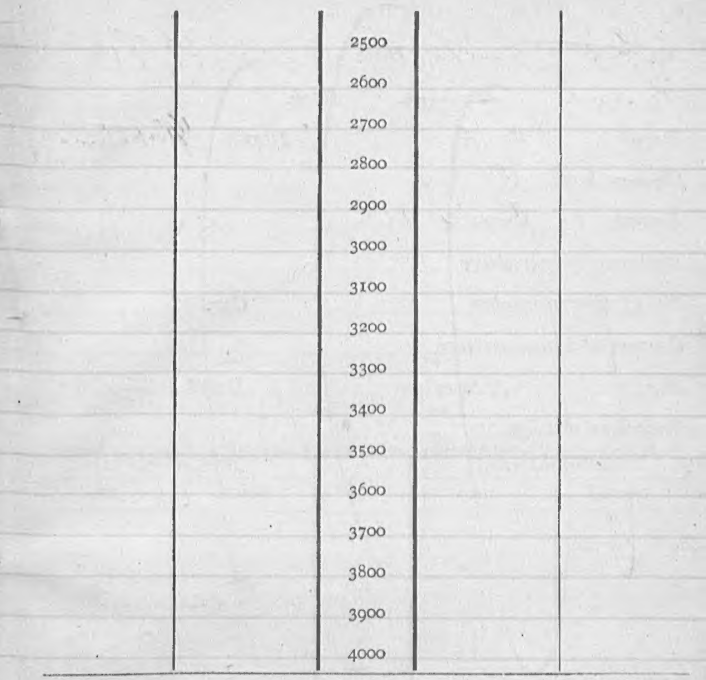

SERIAL TEMPERATURES.

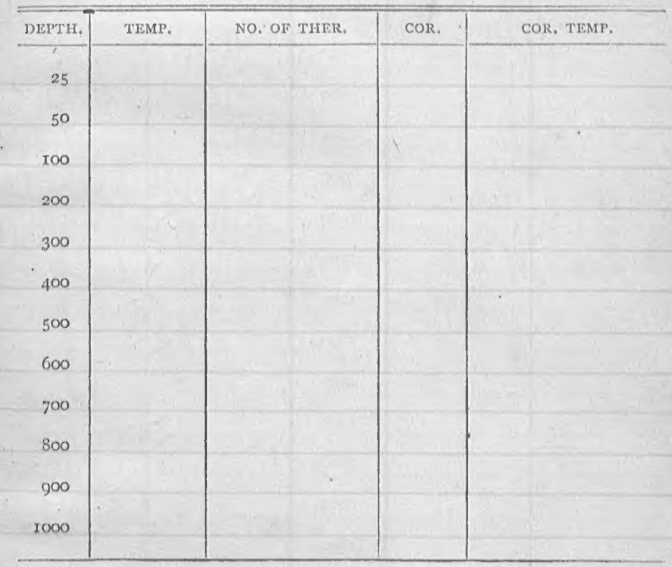

REMARKS : 
507.

295

(1).

of $\sin d$

Turns

Degehine.

(1)

Cor. +

Shotor leald li\& tho

Bottom Corai wih of T \&h.

Bottom temperature

No. of thermometer

Corrected temperature

Air

Silrface

Traw or dredge

AU SOUNDING WIRE

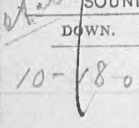

$10-\underbrace{\text { Down. }}_{8}$

(

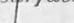

reet.

Depth $y / 2$ Trom

Cor.
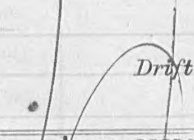

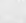

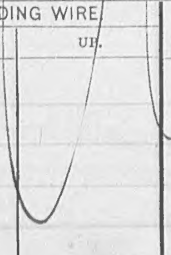

TURN.

DREDGE ROPE.

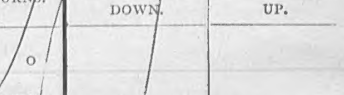

IOO

200

300

500

600

700

800

900

I000

IIOO

1200

1300

1400

I 500

1600

1700

I 800

1900

2000

2100

2200

2300

2400 